\title{
Small-scale structures in the upper atmosphere of the Sun
}

\author{
Dissertation \\ zur Erlangung des mathematisch-naturwissenschaftlichen Doktorgrades \\ "Doctor rerum naturalium" \\ der Georg-August-Universität Göttingen \\ im Promotionsprogramm PROPHYS \\ der Georg-August University School of Science (GAUSS)
}

\author{
vorgelegt von \\ Krzysztof Barczynski \\ aus Wloszczowa, Polen
}

Göttingen, 2017 
Betreuungsausschuss

Prof. Dr. Hardi Peter

Max-Planck-Institut für Sonnensystemforschung, Göttingen, Germany

Prof. Dr. Stefan Dreizler

Institut für Astrophysik, George-August-Universität Göttingen, Germany

Dr. Davina Markiewicz-Innes

Max-Planck-Institut für Sonnensystemforschung, Göttingen, Germany

Mitglieder der Prüfungskommision

Referent: Prof. Dr. Hardi Peter

Max-Planck-Institut für Sonnensystemforschung, Göttingen, Germany

Korreferent: Prof. Dr. Stefan Dreizler

Institut für Astrophysik, George-August-Universität Göttingen, Germany

Weitere Mitglieder der Prüfungskommission:

Prof. Dr. Ansgar Reiners

Institut für Astrophysik, George-August-Universität Göttingen, Germany

Prof. Dr. Wolfgang Glatzel

Institut für Astrophysik, George-August-Universität Göttingen, Germany

Prof. Dr. Ariane Frey

II. Physikalisches Institut - Kern- und Teilchenphysik, George-August-Universität Göttingen, Germany

Prof. Dr. Andreas Tilgner

Institut für Geophysik, George-August-Universität Göttingen, Germany

Tag der mündlichen Prüfung: 11.04.2017 


\section{Contents}

\begin{tabular}{ll}
\hline Summary & 7
\end{tabular}

\begin{tabular}{lll}
\hline & Introduction & 9
\end{tabular}

\begin{tabular}{|lll}
\hline 2 & The solar atmosphere & 13
\end{tabular}

2.1 Overall description of the solar atmosphere . . . . . . . . . . . . 13

2.2 The coronal energy sources $\ldots \ldots \ldots \ldots$. . . . . . . . . . 16

\begin{tabular}{lll}
\hline 3 & Technical introduction & 17
\end{tabular}

3.1 Magnetic field in photosphere . . . . . . . . . . . . . . . . 17

3.2 Formation of the chromospheric lines . . . . . . . . . . . . . . . . 19

3.2.1 The introduction to radiative transfer theory . . . . . . . . . . . . 19

3.2.2 Local Thermodynamic Equilibrium (LTE): . . . . . . . . . . . . 20

3.2 .3 The line formation . . . . . . . . . . . . . . . . 21

3.3 Optically thin emission lines . . . . . . . . . . . . . . 23

$3.3 .1 \quad$ Formation optically thin emission lines . . . . . . . . . . 23

3.3.2 Optically thin emission lines in the solar atmosphere . . . . . . . 23

4 Instrumentation, data preparation and analysis technique $\quad 25$

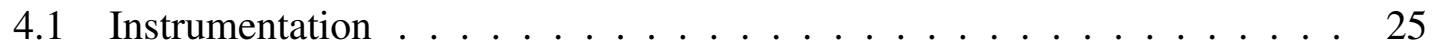

$4.1 .1 \quad$ Photospheric magnetic field measurement (HMI, Hinode) . . . . 25

4.1.2 Observation of the upper solar atmosphere (IRIS, SDO, Hi-C) . . 26

4.2 Data preparation (data levels) $\ldots \ldots \ldots \ldots$. . . . . . . . . . . . . . . . . . . . . . .

4.3 Data analysis technique $\ldots \ldots \ldots \ldots . \ldots . \ldots . \ldots . \ldots 31$

4.3 .1 Differential emission measure (DEM) . . . . . . . . . . . . 31

4.3 .2 Correlation coefficients . . . . . . . . . . . . . . . 32

4.3 .3 Co-aligning . . . . . . . . . . . . . . . 36

$\begin{array}{lll}5 & \text { Miniature loops in the solar corona } & 37\end{array}$

5.1 Introduction . . . . . . . . . . . . . . . 38

5.2 Observations . . . . . . . . . . . . . . . . 40

5.3 Properties of small loop-like features . . . . . . . . . . . . . . . . . 42

5.3 .1 Identification of small loop-like features . . . . . . . . . . . . . . . . . 43

5.3.2 Properties of small loop-like features . . . . . . . . . . . . . . . . . . . . . . . . 46

5.4 Relation to photospheric field . . . . . . . . . . . . . . . . 46

5.5 Relation to thermal structure . . . . . . . . . . . . . . . . . . . 48

5.5 .1 Differential Emission Measure (DEM) . . . . . . . . . . . . . 48 
5.5 .2 X-Ray observations . . . . . . . . . . . . . . . 50

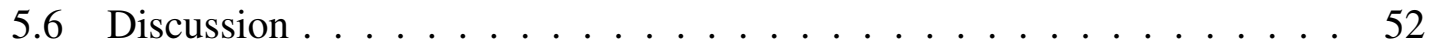

5.7 Conclusions . . . . . . . . . . . . . . . . . . . 53

5.8 Appendix $\ldots \ldots \ldots \ldots \ldots \ldots \ldots \ldots$

5.8 .1 Properties of individual loop-like features . . . . . . . . . . . 54

5.8 .2 Spatial alignment of Hi-C with magnetogram . . . . . . . . . 55

\begin{tabular}{|lll}
\hline & The small-scale structure in the transition region & 59
\end{tabular}

6.1 Introduction . . . . . . . . . . . . . . . . . . 59

6.2 Observation and data preparation . . . . . . . . . . . . . . . 60

6.2 .1 Observations . . . . . . . . . . . . . . . . . . . . . . . . . 60

6.2 .2 Data reduction $\ldots \ldots \ldots \ldots$. . . . . . . . . . . 61

6.3 Descriptions of structures $\ldots \ldots \ldots \ldots$. . . . . . . . . . . 63

6.3 .1 Small-scale loop-like structure . . . . . . . . . . . . . . . . 63

6.3 .2 Two magnetic field patches . . . . . . . . . . . . . . . 67

6.4 Discussion . . . . . . . . . . . . . . . . . . . . . . 68

$6.4 .1 \quad$ Small-scale loop-like structure . . . . . . . . . . . . . . . . 68

6.4 .2 Two magnetic field patches . . . . . . . . . . . . . . . . . . 69

6.4 .3 Conclusion . . . . . . . . . . . . . . . . 70

7 Emission of solar chromospheric and transition region features related to the $\begin{array}{lr}\text { underlying magnetic field } & \mathbf{7 3}\end{array}$

7.1 Introduction . . . . . . . . . . . . . . . . . . . . 74

7.2 Observations and preparation of data $\ldots \ldots \ldots$. . . . . . . . 76

7.2 .1 Observations . . . . . . . . . . . . . . 76

7.2 .2 Data reduction . . . . . . . . . . . . . . . . . . . . . . . . . . .

7.2 .3 Regions of interest . . . . . . . . . . . . . . . 80

7.3 Methods . . . . . . . . . . . . . . . . . . . 82

7.3 .1 Basal flux . . . . . . . . . . . . . . . 83

7.3.2 Relating the upper atmosphere emission to the surface magnetic field: mag-flux relations . . . . . . . . . . . . . 84

7.3 .3 Flux-flux relations $\ldots \ldots \ldots \ldots$

7.4 Results . . . . . . . . . . . . . . . . . . . . 87

7.4 .1 Active region (without sunspots and pores) . . . . . . . . . . 87

7.4 .2 The plage area excluding the quiet Sun . . . . . . . . . . . . . . 91

7.4.3 Quiet Sun and coronal hole . . . . . . . . . . . . . . . . 91

7.5 Discussion . . . . . . . . . . . . . . . . 92

7.5 .1 Correlation between magnetic field and emission . . . . . . . . 92

7.5.2 Mag-flux relation from the photosphere to the chromosphere . . . 94

7.5.3 Mag-flux relation from the chromosphere to the transition region. 96

7.5.4 Mag-flux relation at different resolutions and regions . . . . . . . 97

7.5 .5 Flux-flux relations: the Sun and stars . . . . . . . . . . . . 98

7.6 Conclusion . . . . . . . . . . . . . . . . . . . . . . . 99

7.7 Appendix $\ldots \ldots \ldots \ldots$. . . . . . . . . . . . . . . . . . . . 100

7.7 .1 Basal flux calculation . . . . . . . . . . . . . . 100

7.7.2 Comparing different methods to determine power-law relations . . 102 
\begin{tabular}{lll}
\hline & Summary and outlook & 105
\end{tabular}

\begin{tabular}{lr}
\hline Bibliography & 109
\end{tabular}

\begin{tabular}{lr}
\hline Publications & 119
\end{tabular}

\begin{tabular}{|r|r|}
\hline Acknowledgements & 121
\end{tabular}

\begin{tabular}{lr}
\hline Curriculum vitae & 123
\end{tabular} 



\section{Summary}

Context: Numerous small-scale structures with sizes of the order of megameters $(\mathrm{Mm})$, constitute the background for the large-scale structures in the solar atmosphere. Although these features are small, their large number suggests that they play an important role in the energy transport and the magnetic structuring in the solar atmosphere.

Aims: The aim of this work is to investigate the physical properties of the small-scale structures in the solar atmosphere. Particular attention is given to miniature loops (with a length of approximately $1 \mathrm{Mm}$ ) observed for the first time at coronal temperature ( $>$ $1 \mathrm{MK})$, and their relation between the emission of the small-scale structures and the underlying magnetic field. Many of the small-scale structures in the solar atmosphere remain unresolved with current telescopes. Still, one can investigate the relation of the small-scale structures to the underlying magnetic field in a statistical fashion. This will be the final topic of this thesis.

Methods: We focus here on UV and EUV observations, in combination with photospheric magnetic field maps (magnetograms) from space-based telescopes. The corresponding images, magnetograms and spectroscopic raster maps are aligned to sub-pixel accuracy. The spectral data are used to derive various plasma parameters (e.g. intensity, Doppler shift) by line-fitting techniques, and create the respective 2D maps. Further more, we employ a Differential Emission Measure (DEM) technique to estimate coronal temperatures from the EUV images. Finally, the spatial and temporal evolution of structures is compared with changes of the underlying photospheric magnetic field.

Results: The small-scale structures that are analysed in this thesis have a broad range of lifetimes, from few minutes to several tens of minutes. In particular, the miniature loops have a lifetime compatible to that of granules ( $\approx 5$ minutes), whereas the magnetic field patches exist for about $1 \mathrm{hr}$. We argue that the small-scale short-living structures can reach coronal temperatures. Further more, the structures are closely related to the photospheric magnetic field. A statistical analysis of small-scale upper atmospheric structures in various magnetically active regions shows that the emission from these structures can be used as a proxy for the magnetic field. By assuming a power-law relation of the emission to the magnetic field, we show that this power-law varies smoothly from the lower chromosphere to the transition region.

Conclusion: The result of this thesis reveals a new view of the small-scale structures in the solar atmosphere. We suggest that small-scale loop-like structures observed at coronal temperatures are miniature versions of hot coronal loops. Based on the statistical studies we conclude that the emission originating from higher temperatures is more sensitive to the magnetic field. The decrease of the correlation between magnetic field and intensity with temperature is probably caused by the spatial expansion of the magnetic structures. 



\section{Introduction}

Approximately 4.6 billion years ago in the Local Group, in a galaxy named the Milky Way a new star, the Sun, was formed from a molecular cloud. The Sun is located in the Orion Arm approximately $8.35 \mathrm{kpc}$ from the Galactic Center. Although the Sun is a mediocre object on the main sequence, with luminosity class $\mathrm{V}$ and spectral type $\mathrm{G} 2$, it is also the Earth's closest star $\left(1.496 \times 10^{11} \mathrm{~m}\right)$. It is a ball of gas of $6.96 \times 10^{8} \mathrm{~m}$ radius. This size and close distance imply an angular size in the ground-based observation of about $32^{\prime}$. The luminosity of about $3.8 \times 10^{26} \mathrm{~W}$ and the close distance to the Earth makes the Sun the brightest object in the sky.

The nature of the Sun was, for a long time, a mystery. The oldest notes about the solar eclipse observation have been prepared in 2136 BC in China. In the sixteenth century Copernicus (1543) stated that the Sun is in the center of the Solar System. Since the ancient time dark spots were observed on the solar disk. In the seventeenth century Galileo Galilei used a telescope, for the first time in solar research, and obtained a series of sunspots observations. The long-term observation shows that the number of sunspots changes periodically as noticed by Schwabe (1844). These studies initiated the investigation of the solar cycle, where the sunspot number is used as a proxy of solar activity. William Hyde Wollaston discovered the dark features in the solar spectrum that Fraunhofer rediscovered and found 570 of that dark lines. Kirchhoff concluded that they are absorption lines created in the solar atmosphere. It was a fundamental work for the development of the spectroscopic observation that allows to investigate the physical properties of the solar atmosphere. When Hale (1908) discovered the magnetic nature of sunspots it was clear that the magnetic field plays an important role in the physics of the Sun.

However, the solar atmosphere hides more secrets. Grotrian (1939) and Edlén (1943) observed the emission lines of highly ionized atoms (Fe XIv, Ca xv, Fe x). They suggested that the temperature in the outer part of the solar atmosphere, the solar corona, is significantly higher (more than $1 \mathrm{MK}$ ) than in low-lying photosphere (around 6000K). The question of 'how the solar corona is heated?' called the coronal heating problem was carefully analysed by several generations of solar physicists. Many heating mechanisms were considered, the most famous are the magnetohydrodynamics waves (Alfvén 1947), acoustic waves (Schwarzschild 1948, Biermann 1948) and magnetic field reconnection (Parker 1972). Although space-based observations open new possibilities in solar atmosphere studies, many questions related to coronal heating still remain unanswered.

The new generation of space-based observatories such as Solar and Heliospheric Observatory (SOHO), Solar Terrestrial Relations Observatory (STEREO), Solar Dynamics Observatory (SDO), Hinode, and Interface Region Imaging Spectrograph (IRIS) allow to investigate the Sun with high-spatial, temporal and spectral resolution as well as provide long-term monitoring of the solar activity. The high-resolution data from these instru- 
ments open a door to investigate small-scale structures that were earlier unresolved. In this thesis the small-scale structures are defined as features with size of the order of granules, in other words of the order of megameters (Mm).

Naturally, the question arises why the investigation of the small-scale structures is so important. The study of the physical properties of small-scale structures allows us to better understand processes that govern the solar atmosphere. The numerous smallscale structures create a background for large structures, which occur less frequently. In the non-sunspot area of the solar photosphere we can observe bipolar small-scale magnetic field structures with separation of approximately $2^{\prime \prime}$ (Centeno et al. 2007). They may correspond to a low-lying magnetic loop that can emerge (Ishikawa et al. 2008, Martínez González and Bellot Rubio 2009) and rise into the upper chromosphere. Therefore, small-scale structures should be considered in the analysis of mass and energy flows. Feldman (1983) suggested that in the transition region small-scale loops are present, some of them are associated with coronal emission (Dowdy 1993). Hansteen et al. (2014) used high-resolution data from IRIS and investigated low-lying loops or loop-segments, with a length of few Mm and with lifetimes of few minutes, at transition region temperatures. These structures are critical to understand the mechanism of the energy transport and heating of the upper solar atmosphere. But can such small loops also get hotter and reach coronal temperature $(1 \mathrm{MK})$ ? The candidates for those structures were for the first time observed by Peter et al. (2013) in data from High-Resolution Coronal Imager (Hi-C). In Chap. 5 we analyse the size, motion, temporal evolution as well as the relation with underlying magnetic field and thermal properties of miniature loops in the solar corona. These studies show that miniature-loops (with a length of $1 \mathrm{Mm}$ ) are probably smaller versions of the normal hot coronal loops. They connect opposite magnetic field polarities in the photosphere and then emerge to the solar corona. This implies that coronal heating in the solar atmosphere is highly structured and the small-scale structures play an important role in the energy transport and the heating mechanism.

In the past three years, other types of small-scale structures were discovered and investigated, for example EUV bright dots (EBDs) (Régnier et al.2014) and penumbral bright dots (Tian et al.2014). These discoveries and previous studies suggest that the small-scale structures exist almost everywhere in the solar atmosphere, from the photosphere to the solar corona. In this thesis, the mutual relation between small-scale structures at different temperatures as well as the relation with the underlying photospheric magnetic field are studied. Finding the interrelationship between them is important to create a global picture of the atmosphere as well as to understand the processes occurring in it. Chap.6 is focused on the connection between small-scale structures in the transition region and the photospheric magnetic field as well as the dynamics of these structures.

Not all the small scale structures are visible even when using modern high-resolution instruments. However, the general study of the relation between emission from different parts of the solar atmosphere can give a feedback on how unresolved structures affect the Sun's atmosphere. To investigate the influence that unresolved structures have on the processes in the solar atmosphere we use statistical methods based on the analysis of the spatial connection between intensity fluxes from the photosphere, chromosphere, and transition region (Chap.77). These fluxes contain the vital information about how the energy is transported upwards in the solar atmosphere. The long-term changes of the solar irradiance due to the change of numerous small-scale structures were investigated 
by Krivova et al. (2003). To obtain better models that explain the solar radiation we must gain a deeper knowledge of the physical properties of those small structures. In addition, the relationship between the upper solar atmospheric emission and the underlying photospheric magnetic field is important for stellar physics. These relationships provide a proxy of stellar activity through the flux seen in spectral lines.

\section{Outline}

First, this thesis provides an overview over of the solar atmosphere and discusses the problem of the coronal heating in Chap.2. The technical introduction, Chap.3, presents a method to measure the photospheric magnetic field. This chapter also provides basic information about line formation in the chromosphere and the upper solar atmosphere. In Chap. 4, introduces the instruments that are used in the analysis. This is followed by a description of the data reduction and the analysis techniques that are used in the next chapters of the thesis. The analysis starts in Chap. 5 with the investigation of the properties of miniature-loop like structures. This is followed by discussion of the smallscale structures in the transition region (Chap.6), especially focusing on the dynamics of these structures as well as the relationship with the underlying photospheric magnetic field. Finally, the focus moves to the global properties of the solar atmosphere that cause the existence of unresolved small-scale structures. Lastly, the flux-flux relations in the solar atmosphere are discussed in Chap.7 and this thesis is concluded in Chap.8. 



\section{The solar atmosphere}

The solar atmosphere is the outer shell of the Sun, composed mainly of ionized gases. The structure of the solar atmosphere is inhomogeneous and usually it is divided into three layers: the photosphere, the chromosphere, and the solar corona. The changes of the physical properties between the chromosphere and the solar corona are so significant that the interface between them, the transition region, is usually analysed separately. The solar atmosphere is a highly dynamic environment with large numbers of structures with different geometrical and physical properties, therefore strict borders between layers do not exist. Still, the layers concept is commonly used, because it allows structures to be grouped. However, on the real Sun the atmosphere is highly dynamic and the different regimes interact strongly by exchange of mass and energy, all moderated by the magnetic field.

In this chapter, the different atmospheric layers and the heating mechanisms of the solar atmosphere are discussed.

\subsection{Overall description of the solar atmosphere}

\section{The photosphere}

The photosphere is the inner part of the solar atmosphere and the main source of the white light from the Sun. The base of the photosphere is defined as the layer where the optical depth at $5000 \AA$ is one. The temperature of photosphere decreases from $6000 \mathrm{~K}$ at the bottom to $4500 \mathrm{~K}$ at the top. The density in the photosphere also decreases and the average value is $2 \times 10^{-4} \mathrm{~kg} \mathrm{~m}^{-3}$. The usual approximation of the photospheric emission is a Planck function with the peak around $5000 \AA$ (green-yellow), that corresponds to an effective temperature $5778 \mathrm{~K}$. The characteristic spectral features for this layer are absorption lines, among the strongest there are the Fraunhofer lines. The conditions in the solar photosphere are governed by convective motions, magnetic field, and p-mode oscillation. The main building blocks of the solar photosphere are granules that are short-lived (5-10 min) and small-scale (around $1 \mathrm{Mm}$ ). One of the most characteristic photospheric structures are sunspots, darker and colder than the surrounding area and related to a high concentration of magnetic field with opposite polarities.

\section{The chromosphere}

The chromosphere is visible by eye only immediately before or after the maximal phase of the total solar eclipse as the reddish glow around the solar disk. In contrast to the photosphere, the temperature in the chromosphere increases reaching $3 \times 10^{4} \mathrm{~K}$ at the top of this layer. The density continuously decreases from $8 \times 10^{-5} \mathrm{~kg} \mathrm{~m}^{-3}$ near the photosphere 
to $10^{-11} \mathrm{~kg} \mathrm{~m}^{-3}$ in the upper chromosphere. The low and middle chromosphere are heated by acoustic waves, the upper part by MHD waves (Bhatnagar and Livingston 2005). The chromospheric spectrum is dominated by lines with strong cores such as $\mathrm{Ca}$ II, $\mathrm{Mg}$ II and $\mathrm{H} \alpha$. The average thickness of the chromosphere in a static one-dimensional model (Vernazza et al. 1981) is around $2300 \mathrm{~km}$. However, the chromosphere is rich in structures and highly dynamic. Therefore the real height can significantly deviate from the average value. The most characteristic structure is the chromospheric network, which is closely related to the cells of the supergranulation. The chromospheric network is observed on the solar disk as a pattern consisting in cells with size of about $20 \mathrm{Mm}$. On the disk the dark mottles are visible, the same structures that are seen as spicules on the solar limb.

\section{The transition region}

The transition region (hereafter, TR) is an interface between the cooler chromosphere $\left(3 \times 10^{4} \mathrm{~K}\right)$ and significantly hotter corona $(1 \mathrm{MK})$. The TR is only few hundreds kilometer thick, but in this region the temperature increases by two orders of magnitude. The density sharply decreases 40 times compared with the upper chromosphere $\left(10^{-11} \mathrm{~kg} \mathrm{~m}^{-3}\right)$. When the temperature rises the structures seen in the solar chromosphere become more smooth and the chromospheric network disappears. The transition region is dominated by small-scale cool loops. In the TR the temperature increases and the less abundant, heavier ions start to dominate in the spectrum, especially in 500 -1600 А wavelength range.

\section{The solar corona}

The solar corona is the outer part of the solar atmosphere. The name comes from Latin and means 'crown'. The corona is visible with naked eye as the faint and irregular halo during a total solar eclipse. Observations in other conditions are difficult because the solar disk is million times brighter than the corona. In 1930 Bernard Lyot built the first coronograph, which is an instrument which permits the observation of the corona by blocking the disk emission using a small occulter. Without blocking the disk emission the solar corona can be observed in EUV and X-ray wavelengths, but only above the Earth's atmosphere. Nowadays, multichannel space-based instruments, for example Solar and Heliospheric Observatory and Solar Dynamics Observatory, provide continuous monitoring of structures in the solar corona.

Coronal structures cover a huge range of sizes, from 1Mm miniature loop to Coronal Mass Ejections (CMEs) that can even cross the orbit of the Earth. The shape and size of these structures are mainly determined by the magnetic field. Areas with strong, opposite magnetic field, rich in coronal loops, X-ray bright points, and X-ray jets are called active regions (AR). The highly dynamic phenomena such as solar flares and CMEs are usually closely related to them. The source of the slow solar wind, the helmet streamers, are also associated with them. The active regions have a higher temperature and emit more

$\mathrm{X}$-ray emission than surroundings. In contrast, the coronal hole is an open magnetic field region, usually existing near the poles. The open magnetic field allows the plasma to escape easily so the coronal holes are the main source of the fast solar wind. Therefore in these regions, the plasma density and temperature are two times lower compared with coronal averages. The area with low magnetic field that is not an active region or coronal hole is called the quiet Sun (QS). The temperature and density in the quiet Sun are closer to the average coronal values. 


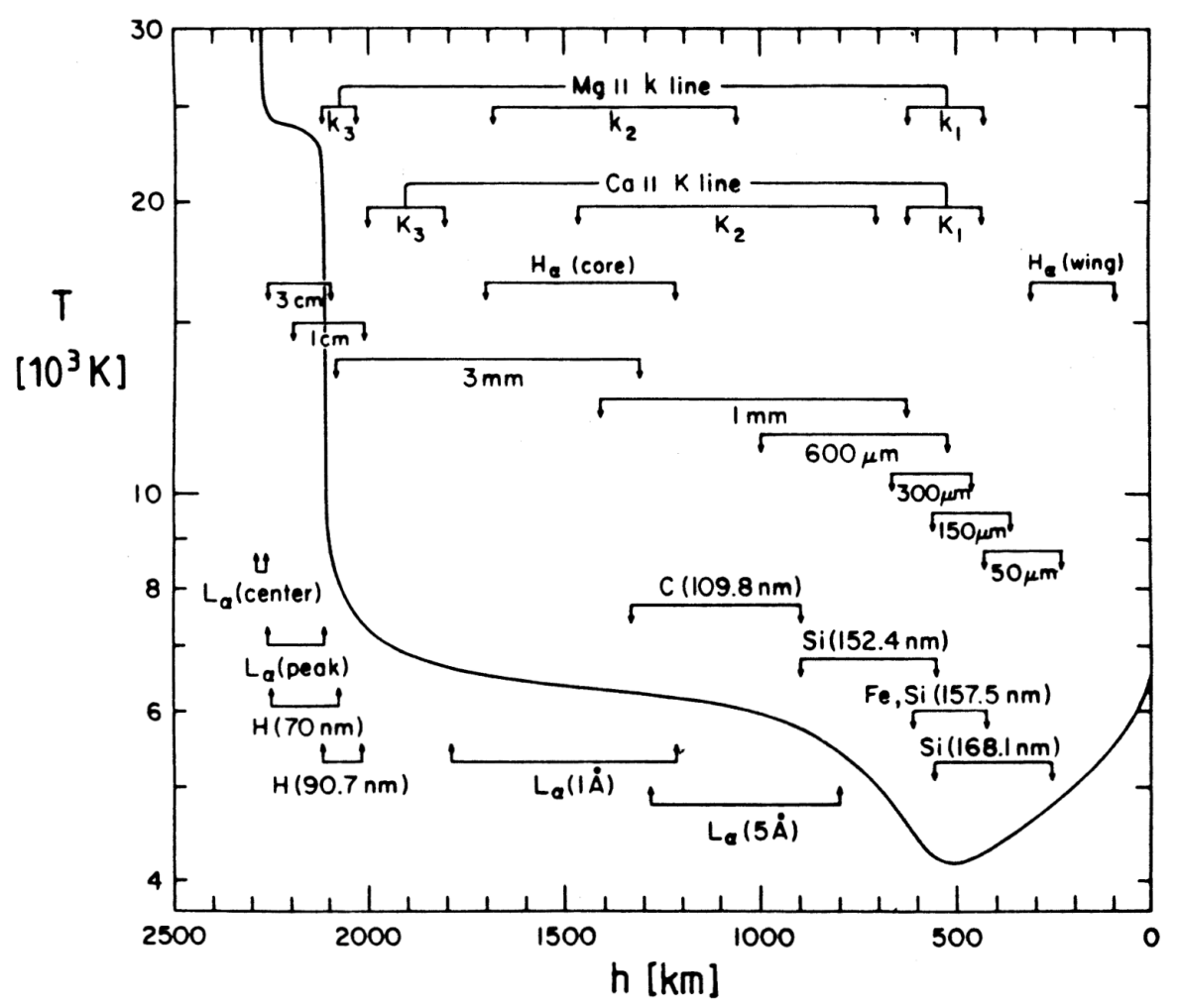

Figure 2.1: The temperature as function of height in the VAL model (Vernazza et al. 1981). The zero height corresponds to the bottom of the solar photosphere. The solid line presents the relationship between temperature and the height. The horizontal lines mark the formation layers of lines and continua in this model.

Above the transition region, the temperature profile is flatter, the average coronal temperature is around $1 \mathrm{MK}$. However, it is distributed non-uniformly and varies highly for different coronal structures, as does the density. The average density is $10^{14} \mathrm{~kg} \mathrm{~m}^{-3}$. The high temperature causes the plasma in the solar corona to be fully ionized. The coronal emission is dominated by highly ionized atoms, for example, from $\mathrm{Fe}$ ix to $\mathrm{Fe} x \mathrm{x}$ which are present from $1 \mathrm{MK}$ to $10 \mathrm{MK}$. It is clear that the temperature of the solar corona is significantly higher than the photosphere, the possible mechanism of the coronal heating is discussed by Klimchuk (2006), however, the coronal heating problem is still an open issue.

\section{The VAL model}

A useful approximation of the mean physical properties of the photosphere and the chromosphere is the VAL model (Vernazza et al. 1981). It models how the temperature varies with height, assuming the base of the solar photosphere is where $\tau=1$ at $5000 \AA$ and the temperature minimum $(4300 \mathrm{~K})$ is $550 \mathrm{~km}$ above the base of the photosphere. Although VAL is a 1D model and does not present the real photosphere and chromosphere it gives an overview of the trend of temperature changes in the solar atmosphere (see Fig.2.1). 


\subsection{The coronal energy sources}

The solar atmosphere is heated by energy generated in the solar core by thermonuclear fusion. This energy is transported towards the surface firstly by radiation (in the radiation zone) and next by convection (in the convection zone). However, the low-lying photosphere $(6000 \mathrm{~K})$ is significantly cooler than the solar corona $(1 \mathrm{MK})$ above. This suggests that an additional source of energy exists. The problem of how the solar corona is heated is not fully solved. An important questions still remain open: which structures or mechanisms have the highest influence for the heating? How is the heating generated?

Several mechanisms of the coronal heating were proposed. They are usually categorized into two groups: DC (Direct Current) and AC (Alternating Current). The DC processes are related to the stresses and reconnection, in other words they are dependent on the magnetic field. Some example are stress-induced reconnection (Sturrock and Uchida 1981) and stress-induced turbulence (Heyvaerts and Priest 1992). The AC processes are related to wave heating processes, for example, the Alfvénic resonance (Hollweg 1991) and MHD turbulence (Inverarity and Priest 1995). Based on these mechanisms, several models try to explain the source of the coronal heating Klimchuk (2006). 


\section{Technical introduction}

The solar spectrum is a key source of information about the physical condition of the solar atmosphere. Spectroscopy is used in diagnostics of the chemical composition as well as temperature and density. The Doppler effect provides information about the velocity of the plasma motions that are parallel to the line-of-sight. The Zeeman effect allows to study the magnetic field is based on polarimetry in spectral lines. In this section we describe measurements of the photospheric magnetic field (Sect. 3.1) as well as some theory of the line formation in the chromosphere (Sect. 3.2) and optically thin plasma medium, the transition region and the solar corona (Sect. 3.3.

\subsection{Magnetic field in photosphere}

The magnetic field in the solar atmosphere covers a wide range of sizes, from small-scale structures such as magnetic bright dots to the large-scale such as coronal holes. The plasma in the solar atmosphere is governed by the magnetic field. Therefore, the analysis of magnetic fields is necessary to understand phenomena and evolution of structures in the solar atmosphere. The magnetic field measurement in the solar atmosphere is based on methods such as Zeeman effect, Hanle effect or gyroresonance. Proxies of magnetic field strength can be also obtained in an indirect way, for example, from the measurement the intensity of the $\mathrm{Ca}$ II $\mathrm{K}$ line. In this chapter, we focus on the Zeeman effect because all magnetograms presented in the thesis are based on this effect.

In 1896 Pieter Zeeman discovered that a static magnetic field causes a splitting in the Fraunhofer D line (sodium doublet at $5890 \AA$ and $5896 \AA$ ) into several components (Zeeman 1897). Theses components are polarized which allows to use a polarimetric method and measure the magnetic field.

On the atomic level, the Zeeman effect is due to the removal of degeneracy from atomic levels through the interaction between the external field and the atomic magnetic moment (Shore 2002). The following description is based on Stix (2004). When taking an analytical approach to that effect, first, we define the state of an atom in term of the orbital angular momentum $(L)$, the spin angular momentum $(S)$, total angular momentum $(J)$ and the magnetic quantum number $\left(M_{J}\right)$. For a weak magnetic fields $(B)$ that satisfies the LS coupling, the change of the energy $\left(E_{J M}\right)$ of the atom is:

$$
E_{J M}=E_{J}+\mu_{0} g M_{J} B
$$

In Eq. 3.1, $E_{J}$ is the energy of the atomic level in absence of the magnetic field, $\mu_{0}=$ $e \hbar /(2 m c)$ is the Bohr magneton ( $m$ is the electron mass rest, $e$ is the elementary charge, 
$c$ is the speed of light), $g$ is the Landé factor. The Landé factor for each state with LS condition is:

$$
g=1+\frac{J(J+1)+S(S+1)-L(L+1)}{2 J(J+1)}
$$

We now consider two cases of the spin angular momentum $S=0$ and $S \neq 0$ that correspond to the normal Zeeman effect and the anomalous Zeeman effect, respectively. In normal Zeeman effect $g$ is one and transition $\Delta M_{J}=-1,0,1$. The spectral line splits into a triplet consisting of two shifted $\sigma$ components (related to $\Delta M_{J}=-1,1$ ) and unshifted $\pi$ components (related to $\Delta M_{J}=0$ ). The difference of wavelengths between the peak of the line center and the peak of the $\sigma$ component $(\Delta \lambda$, eq. 3.3$)$ is proportional to a wavelength $(\lambda)$ of the center peak and the magnetic field $(B)$ :

$$
\Delta \lambda \sim \lambda^{2} B
$$
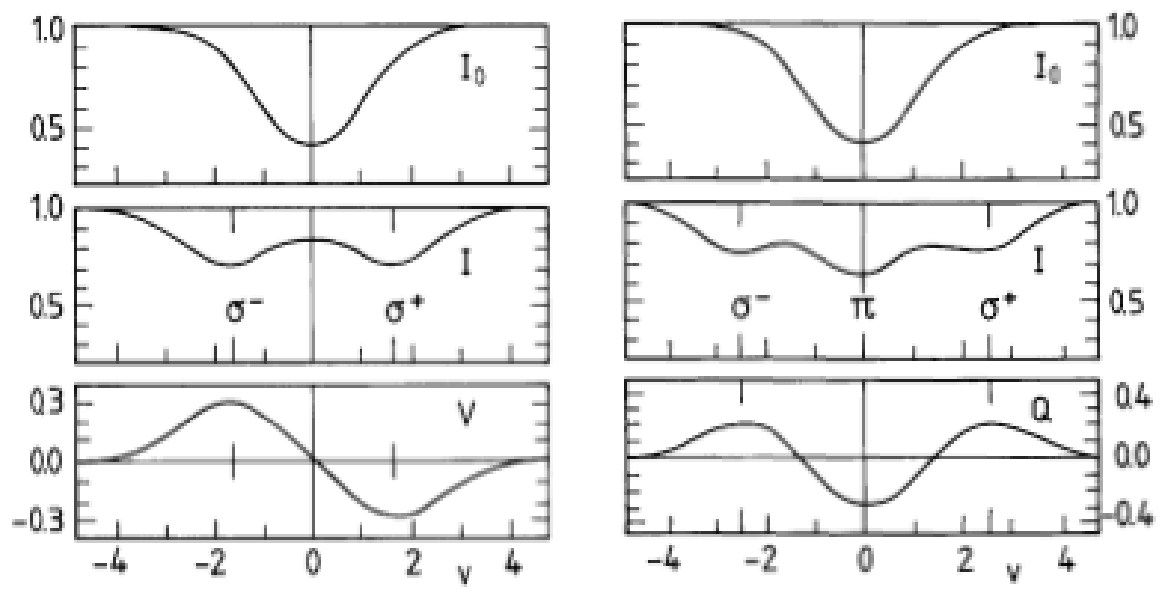

Figure 3.1: The characteristic profile of the the Stokes parameter of the absorption line with longitudinal magnetic field (left) and transverse magnetic field (right). $I_{0}$ is the profile with no magnetic field. $I$ is the intensity profile with magnetic field that $V$ is the difference of left and right circular polarization. $Q$ is difference between linear polarizations (Stix 2004).

The intensity profile across the spectral line depends on the geometrical configuration of the line-of-sight (LOS) and the magnetic field. In the longitudinal Zeeman effect, the magnetic field is parallel to LOS, then only the $\sigma$ components are visible. They have a circular polarization with an opposite sign. The $\pi$ component disappears (see left panel of Fig. 3.1). This description is valid for emission and absorption lines. In transverse Zeeman effect, the magnetic field is perpendicular to the LOS then one $\pi$ and two $\sigma$ components are observed, all with linear polarization (see right panel of Fig. 3.1). In the case of emission line, the $\sigma$ components are perpendicular to $B$ and $\pi$ is parallel to $B$ (the opposite for absorption lines).

The normal Zeeman effect is really rare. The normal case is the anomalous Zeeman effect, when $S \neq 0$ and $g$ is calculated from Eq. 3.2 with proper quantum numbers. The 
polarization rules for normal and anomalous Zeeman effect are the same. In the observations of the magnetic field in the solar photosphere heavily used lines are Fe I (6173 $\AA$ ) or Ni I $(6768 \AA)$.

\subsection{Formation of the chromospheric lines}

This subsection discusses the formation of chromospheric lines. To this end, a brief introduction to the radiative transfer equation is presented. Then the concept of the Local Thermodynamic Equilibrium (LTE) is discussed. Finally, chromospheric lines are described, especially Mg II h\&k. A more detail introduction to the radiative theory is provided by Rybicki and Lightman (1979). A description of the line formation in the solar chromosphere can be found e.g. Stix (2004).

\subsubsection{The introduction to radiative transfer theory}

The intensity, $I_{v}\left[\mathrm{~W} \mathrm{sr}{ }^{-1} \mathrm{~m}^{-2} \mathrm{~Hz}^{-1}\right.$ ], is one of the key quantities in the study of radiative theory. It is defined (eq. 3.4) as the amount of energy $d E$ crossing an area $d A$ within a frequency range $d v$ in a time $d t$. The inclination $(\theta)$ between the line of sight and the normal of $d A$ is accounted for by a factor $\cos \theta$.

$$
I_{v}=\frac{d E}{\cos \theta d A d t d \Omega d v}
$$

Emission and absorption are responsible for increase and decrease of the intensity. The added intensity (eq. 3.5) is proportional to the spontaneous emission coefficient, $j_{v}$ $\left[\mathrm{W} \mathrm{sr}{ }^{-1} \mathrm{~m}^{-3} \mathrm{~Hz}^{-1}\right]$, and the path between source and observer (s):

$$
d I_{v}(s)=j_{v}(s) d s .
$$

The specific intensity is reduced by absorption. The specific intensity subtracted (eq. 3.6) from the beam is proportional to the intensity, the absorption coefficient, $\alpha\left[\mathrm{m}^{-1}\right]$, and a distance between source and observer $(s)$ :

$$
d I_{\nu}(s)=-I_{v}(s) \alpha(s) d s .
$$

The ratio between emission and absorption is called the source function, $S_{v}\left[\mathrm{~W} \mathrm{sr}^{-1} \mathrm{~m}^{-2} \mathrm{~Hz}^{-1}\right]$, (eq. 3.7):

$$
S_{v}=\frac{j_{v}}{\alpha_{v}} .
$$

The relation between added and subtracted specific intensity is described by the radiative transfer equation (eq. 3.8), where $\mu=\cos \theta$ :

$$
\mu \frac{d I_{v}}{d s}=-\alpha_{v} I_{v}+j_{v} .
$$

Usually in astrophysics we use the optical depth $\tau$ instead of the path length $s$. These two quantities are related by:

$$
d \tau_{v}=-\alpha_{v} d s
$$


The optical depth is dimensionless. The medium with $\tau>1$ is called optically thick (opaque), while for $\tau<1$ the medium is optically thin (transparent). The transfer equation (eq.3.8 can be rewritten in terms of the optical depth and the source function:

$$
\mu \frac{d I_{v}}{d \tau}=I_{v}-S_{v}
$$

The formal solution of this transfer equation is:

$$
I_{\nu}\left(\tau_{v}, \mu\right)=I_{\nu}\left(\tau_{0}, \mu\right) e^{\frac{-\left(\tau_{0}-\tau_{v}\right)}{\mu}}+\frac{1}{\mu} \int_{\tau_{v}}^{\tau_{0 v}} S\left(\tau_{v}^{\prime}\right) e^{\frac{-\left(\tau_{v}^{\prime}-\tau_{v}\right)}{\mu}} d \tau_{v}^{\prime}
$$

where is $\tau_{0 v}$ the optical depth at the reference level.

Assuming $S=$ const and $\mu=1$ we obtain:

$$
I_{\nu}\left(\tau_{\nu}\right)=I_{v}(0) e^{-\tau_{v}}+S_{v}\left(1-e^{-\tau_{v}}\right) .
$$

The solution of the transfer equation depends on the optical thickness. Two extreme cases are presented below:

- optical thick medium: $\tau \mapsto \infty \Rightarrow I_{v}=S_{v}$;

- opitical thin medium: $\tau \mapsto 0 \Rightarrow I_{v}=I_{v}\left(\tau_{v}\right)+\left[S_{v}-I_{\nu}\left(\tau_{v}\right)\right] \tau_{v}$.

The solution of the transfer equation for an optically thick medium is independent of the optical depth (e.g. for the black-body radiation). In an optically thin medium $(\tau<1)$ the relation between $I_{v}\left(\tau_{v}\right)$ and $S_{v}$ determine the emission and absorption under the following conditions:

- emission line: $I_{v}\left(\tau_{v}\right)<S_{v}$ or $I_{v}\left(\tau_{v}\right)=0$;

- absorption line: $I_{v}\left(\tau_{v}\right)>S_{v}$.

The information in this subsection is based on the Rybicki and Lightman (1979) and Stix (2004).

\subsubsection{Local Thermodynamic Equilibrium (LTE):}

Before we apply the radiative transfer equation to describe line formation in the chromosphere we must discuss the thermal properties of the solar atmosphere, especially the concept of Local Thermodynamic Equilibrium (LTE).

Thermodynamic equilibrium (TE) is a state where the entire medium has the same temperature. In other words, a single value of temperature is sufficient to characterize the medium. At that temperature, particles have a Maxwellian velocity distribution at that temperature, ionization and excitation states satisfy Saha and Boltzmann equations for that temperature and the radiation field is a thermal black-body radiation which is described by the Planck function (eq. 3.13):

$$
B_{v}=\frac{2 h v^{3} / c^{2}}{\exp (h v / k T)-1}
$$


again with the same temperature.

In the solar atmosphere the temperature changes from approximately $6000 \mathrm{~K}$ in the photosphere to more than $1 \mathrm{MK}$ in the solar corona. This implies that solar atmosphere is not in thermal equilibrium. However, locally this is the case, at least in the photosphere. This state is called the Local Thermodynamic Equilibrium (LTE). In LTE the source function is expressed by Planck function (eq. 3.14):

$$
S_{v}=B_{v}
$$

In the rarefied medium, the mean free path of particles is larger than a distance of the temperature changes. This state is called non-Local Thermodynamic Equilibrium (nonLTE or NLTE). The NLTE condition usually exists in the core of the strong lines.

Otherwords, in the LTE condition temperatures of radiation $\left(T_{r a d}\right)$, excitation $\left(T_{e x}\right)$, ionization $\left(T_{i o n}\right)$ and gas temperature $\left(T_{\text {gas }}\right)$ are the same, the excitation and ionization states satisfy the Boltzmann and Saha equation. In the non-LTE condition at least one of temperatures is different than rest, for example in the solar corona the excitation temperature $\left(T_{e x}\right)$ are not with equilibrium with gas $\left(T_{\text {gas }}\right)$ and ionization $\left(T_{i o n}\right)$ temperature. Additionally, in the solar corona the states of ionization of the atom does not satisfy the Saha equation.

Information presented in this subsection is based on $\operatorname{Stix}(2004)$.

\subsubsection{The line formation}

The photospheric emission covers the spectral range from the UV $(\lambda>1600 \AA)$ to the IR $(\lambda<100 \mu \mathrm{m})$. The usual approximation is a black-body radiation, with a temperature at $5778 \mathrm{~K}$. The solar photosphere is optically thick and satisfies the LTE conditions, however in this layer the temperature decrease with height. Therefore, the Earth and the spacebased observer sees that cooler material cover the underlying hotter part of the solar photosphere. In that condition, the cooler material absorbs photons of some wavelength from the photospheric continuum emission and creates absorption lines (Fraunhofer lines).

In the chromosphere, the temperature increases with height in a result of non-thermal heating. In that condition, one of the strongest chromospheric line, $\mathrm{H} \alpha(6563 \AA)$, is formed. The $\mathrm{H} \alpha$ emission is the main source of the reddish light observed immediately before or after the maximal phase of the total solar eclipse. The spectroscopic observation of the solar disk in $\mathrm{H} \alpha$ line shows a deep absorption profile, however above the solar limb it turns into emission. This suggests that the chromosphere is mostly transparent for the photospheric radiation, but the $\mathrm{H} \alpha$ line is still opaque and creates an absorption profile in solar disk observations.

The Ca II H\&K (3969 ̊ and 3934 A respectively) and Mg II h\&k (2803 A and $2796 \AA$ respectively) lines play an important role in diagnostics of the solar chromosphere. They are strong, collisional controlled, resonance lines with similar spectral profiles showing an emission reversal. However, the chemical elements that create these lines have different abundances 1 , magnesium $\left(\log \epsilon_{M g}=7.60 \pm 0.04\right.$ ) has larger abundance than calcium $\left(\log \epsilon_{C a}=6.34 \pm 0.04\right)$ (Asplund et al. 2009). This implies that $\mathrm{Mg}$ II is formed higher

\footnotetext{
${ }^{1}$ The abundance is defined as $\log \epsilon_{X}=\log \left(N_{X} / N_{H}\right)+12$, where the hydrogen abundance is $\log \epsilon_{H}=12$ (Asplund et al. 2009).
} 


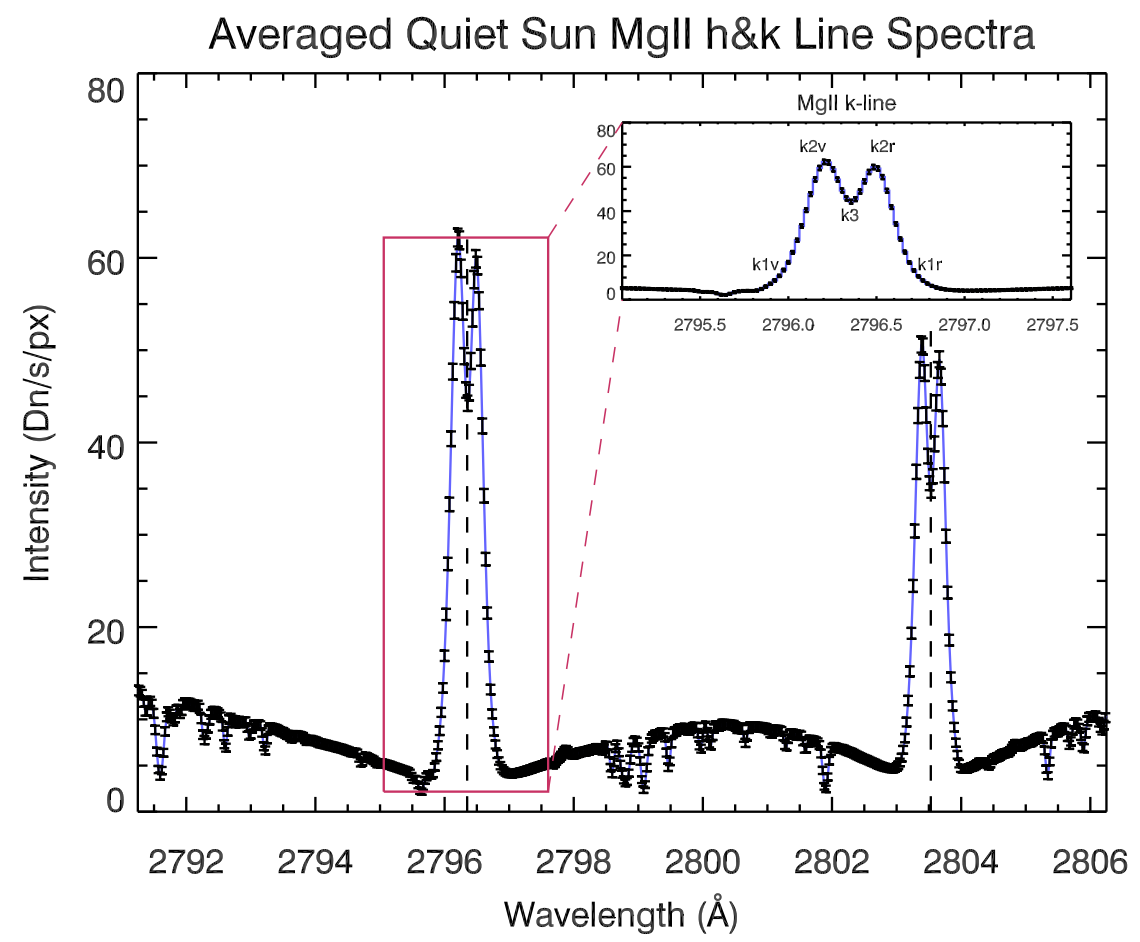

Figure 3.2: The average spectrum of the $\mathrm{Mg}$ II h\&k line observed by IRIS (Kerr et al. 2015). On the reversal profile of the quiet Sun are marked the intensity minimum (k1), the emission peak or emission maxima (k2) and the line center or central deep (k3). The $\mathrm{k} 1$ and $\mathrm{k} 2$ points located on the left or on the right respect to the $\mathrm{k} 3$ points are called violet (thereafter $\mathrm{v}$ ) and red (thereafter $\mathrm{r}$ ), respectively.

in the chromosphere than Ca II (Leenaarts et al. 2013b). Figure 3.2 presents a profile of $\mathrm{Mg}$ II $\mathrm{k}$ line with the emission reversal. When the cooler medium covers the medium with higher temperature the observer sees the absorption profile as in $\mathrm{Mg}$ II wings. For the $\mathrm{Mg}$ II spectral line the emission created at higher temperatures is closer to the line center. Therefore, intensity minima (k1) correspond to the temperature minimum at the upper photosphere and the lower chromosphere. In the chromosphere the temperature increases with height, therefore the cooler photospheric medium is covered by the hotter chromospheric medium. The absorption in this cooler material can be so significant that radiation from hotter layers begins to be visible as a clear emission profile. Moving up towards higher temperature the intensity increases. This corresponds with the emission profile between $\mathrm{k} 1$ and $\mathrm{k} 2$. Moving further up the temperature continuously increases and the density decreases so LTE conditions are not satisfied. The line center (k3) originates under such non-LTE conditions.

The $\mathrm{Mg}$ II and $\mathrm{Ca}$ II lines are used to investigate the thermal properties of the upper photosphere (k1), mid chromosphere (k2) and upper chromosphere (k3) as well as to study the plasma velocity in the chromosphere. They are also used as a proxy of solar and stellar activity. 


\subsection{Optically thin emission lines}

\subsubsection{Formation optically thin emission lines}

The formation of many optically-thin emission lines can be simplified with the two-level approximation. We assume a two-level atom with ground state (level 1) and an excited state (level 2) and the number density of atoms $n_{1}$ at ground state and $n_{2}$ at excited state. The rate for the collisional excitation from level 1 to level 2 is $n_{e} C_{12}$, where $n_{e}$ is the electron density, $C_{12}$ is the collisional rate coefficient. The excitation of atom is followed by spontaneous emission with probability $A_{21}$ (Einstein's A -coefficient) and provides $h v$ energy for each emitted photon. Therefore the total emissivity, $\varepsilon\left[\mathrm{W} \mathrm{m}^{-3}\right]$, generated from spontaneous emission is:

$$
\varepsilon=h v n_{2} A_{21}=n_{e}^{2} G\left(T, n_{e}\right) .
$$

In eq. 3.15 the total energy is expressed by the contribution function $G\left(T, n_{e}\right)$ :

$$
G\left(T, n_{e}\right)=h v \frac{A_{21} n_{2}}{n_{e} n_{1}} \frac{n_{1}}{n_{\text {ion }}} \frac{n_{\text {ion }}}{n_{e l}} \frac{n_{e l}}{n_{H}} \frac{n_{H}}{n_{e}} .
$$

It describes the number density of atoms in level 2 by the relative population of the excited level $\left(n_{1} / n_{\text {ion }}\right)$, relative abundance of the ionic species $\left(n_{\text {ion }} / n_{e l}\right)$, abundance of element relative to hydrogen $\left(n_{e l} / n_{H}\right)$, ratio of the number density of hydrogen to number density of electrons $\left(n_{H} / n_{e}\right)$ (Mariska 1992). If the processes of excitation and deexcitation are in equilibrium, the balance between two processes reads as:

$$
n_{1} n_{e} C_{12}=n_{2} A_{21} .
$$

This implies that $G\left(T, n_{e}\right)$ depends on the collisional rate coefficient:

$$
C_{12}=\frac{A_{21} n_{2}}{n_{e} n_{1}} \sim \frac{1}{T^{1 / 2}} \exp \left(\frac{-\Delta E}{k T}\right) .
$$

It can be approximate as a function of the transition energy between levels $(\Delta E)$ and temperature $(T)$. For more details about this approximation see Mariska (1992).

Typically $n_{e} C_{12}$ is of the order of $1 \ldots 100 \mathrm{~s}^{-1}$ and $A_{21}$ is of the older of $10^{6} \mathrm{~s}^{-1}$ for normal allowed transitions from eq. 3.18 then follows that $n_{1} / n_{2} \gg 1$ and thus that almost all ions are in the ground state $\left(n_{1} / n_{\text {ion }} \approx 1\right)$. The $n_{\text {ion }} / n_{e l}$ is a function of the temperature and depends of species. Each chemical element has a characteristic abundance $\left(n_{e l} / n_{H}\right)$ as presented by e.g. Anders and Grevesse (1989). $n_{H} / n_{e}$ is approximately 0.8 assuming the complete ionization (Mariska 1992).

\subsubsection{Optically thin emission lines in the solar atmosphere}

In a one-dimensional picture of the transition region, the temperature significantly increases and density drops with height. This rarefied and hot medium is a rich source of optically-thin emission lines observed in the UV and EUV parts of the spectrum.

Below $1400 \AA$, the solar spectrum is dominated by emission lines. Above $1400 \AA$ the background solar continuum increases and above $1600 \AA$ only the strongest lines are visible, such as Si II (1817 $)$ ). In this wavelength range, the intensity of the He I $(504 \AA)$ and 


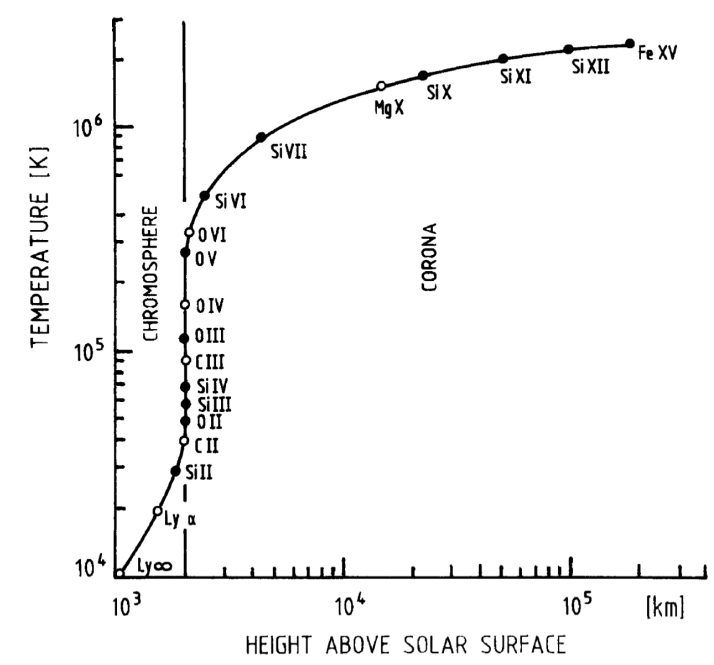

Figure 3.3: Temperature as function of height above the solar surface in the mean model of the solar atmosphere (Reeves et al. 1977). Temperature of the line formation are marked by dots.

Lyman (912 $\AA$ ) continua is comparable with lines intensities. Other continua such as $\mathrm{C}$ I, $\mathrm{S}_{\mathrm{I}}$ and Si I are weaker (Mariska 1992). Spectral lines that form below $1600 \AA$ represent all species in the solar atmosphere, the most abundant of them are $\mathrm{H}, \mathrm{He}, \mathrm{C}, \mathrm{N}, \mathrm{O}, \mathrm{Ne}$, $\mathrm{Mg}, \mathrm{Si}$, and $\mathrm{S}$ with several ionization states. One characteristic for that wavelength range is the series of the Lyman lines (Ly). The strongest of them, Ly- $\alpha(1216 \AA$ ), is the main source of the radiative losses in the upper chromosphere (Lemaire et al. 1998). Other members of that family as $\mathrm{Ly}-\beta(1026 \AA)$ and Ly- $\gamma(972 \AA)$ are also used in the study of the solar atmosphere. The multiplet of $\mathrm{C}$ II lines (around $1335 \AA$ ) is used in diagnostic of the upper chromosphere and lower transition region Rathore et al. (2015). The multiplet systems of O Iv lines and Si Iv (both around $1400 \AA$ ) play an important role in a diagnostics of the plasma density in the transition region and are covered by the recent extreme UV spectrograph and imager IRIS. The highly ionized lines such as Fe xIv, Ca xv and others exist in the solar corona at higher temperatures. The most characteristic optically thin lines which are formed in the upper solar atmosphere are pictured Fig.3.3, together with relation between the average height above the solar surface and their line formation temperature. 


\section{Instrumentation, data preparation and analysis technique}

\subsection{Instrumentation}

Space-based observatories opened a new era for the investigation of the upper solar atmosphere, giving access to EUV and UV wavelength ranges which are not observable for the ground. The EUV and UV lines are one of the main sources of information about conditions of the upper solar atmosphere.

To study the dynamics of small-scale structures, we need data obtained with high spatial and temporal resolution. The magnetic filed measurements are necessary to understand how small-scale structures are related to the underlying magnetic field. The simultaneous observations at different wavelengths (imaging and spectroscopy) give us the possibility to better understand the properties of the structures. Therefore, we decided to use data obtained by four space-based observatories, because these data together satisfy the conditions presented above.

Here, we present informations about the instruments which we used to acquire the data analysed in this thesis. In Sect.4.1.1 we discuss the technical aspects of instruments used to measure the photospheric magnetic field. The instruments used to study the emission from the upper part of the solar atmosphere are presented in Sect.4.1.2.

\subsubsection{Photospheric magnetic field measurement (HMI, Hinode)}

To investigate the magnetic field, in our analysis, we use the line-of-sight magnetograms obtained by SDO/HMI and Hinode/NFI.

\section{SDO/HMI}

The Solar Dynamics Observatory (SDO) was launched on 11 February 2010 from Cape Canaveral. The mission was designed to monitor the solar atmosphere with high spatial and temporal resolutions at several wavelengths simultaneously. SDO also provides information about the photospheric magnetic field and extreme ultraviolet spectral irradiance. The observatory consist of three instruments: Atmospheric Imaging Assembly (AIA), Extreme Ultraviolet Variability Experiment (EVE), and Helioseismic and Magnetic Imager (HMI). The circular geosynchronous orbit inclined by 28 degrees allows near-continuous observation of the Sun.

The Helioseismic and Magnetic Imager (HMI; Scherrer et al. 2012) onboard of SDO measures the photospheric line-of-sight magnetic field, vector magnetic field as well as 
the Doppler shift and continuum intensity. The instrument is designed as filtergraphpolarimeter. The optical system is composed by the refracting telescope with $14-\mathrm{cm}$ aperture, filters, and a polarization-selection mechanism. In this thesis the level-2 data of the line-of-sight magnetograms are used. These provide the component of the magnetic field along the line-of-sight with $45 \mathrm{~s}$ cadence. HMI observes the full solar disk with a plate scale of $0.5^{\prime \prime}$ pixel $^{-1}$ (two-pixel resolution 1.2" pixel $^{-1}$ ). The observation are provided in the $\mathrm{Fe}$ I line at $6173.3 \AA$ over a wavelength range of $76 \mathrm{~m} \AA$. The noise level of the magnetic field measurement is between 5 and $10 \mathrm{G}$. HMI is used to study solar oscillations and the photospheric magnetic field. The information presented here is based on HMI instrument the paper by Scherrer et al. (2012).

\section{Hinode/NFI}

Here we present information about Hinode based on Tsuneta et al. (2008). Hinode (Solar -B) was launched on 22 September 2006 from Uchinoura Space Center in Japan. The main goals of the mission are: understand the influence of the solar magnetic field on the luminosity; study the processes responsible for generation and transport of the solar magnetic field as well as energy transport in the solar atmosphere and coronal heating. The Hinode data also help to find an answer for the question of how eruptive phenomena in the solar atmosphere have an influence on the space weather. The satellite is placed in a the circular, Sun-synchronous orbit at an altitude of $680 \mathrm{~km}$ with an inclination of $98.1 \mathrm{deg}$. Hinode has an orbital period of 98 minutes. This orbit configuration allows to observe the solar disk for nine months per year without interruption. On board of Hinode, three instruments are installed: Solar Optical telescope (SOT), EUV Imaging Spectrometer (EIS) and X-Ray Telescope (XRT). In Chap.6, we use data from SOT.

The Solar Optical Telescope is composed of two blocks: the Optical Telescope Assembly (OTA) and the Focal Plane Package. The OTA is a 50-cm Gregorian telescope with a $400 " \times 400 "$ field-of-view. The Focal Plane Package is a system of a Narrowband Filter Imager (NFI), the Broadband Filter Imager (BFI) and the Spectro-Polarimeter (SP). We use only data from NFI. It provides observations of the solar photosphere and chromosphere in terms of intensity, Dopplergrams and maps of all Stokes parameters (I, Q, U, and V) at six spectral channels between $5172 \AA$ and $6563 \AA$. The NFI has a spectral resolution of $60-100 \mathrm{~m} \AA$ and provides a $328^{\prime \prime} \times 164^{\prime \prime}$ field-of-view with a spatial scale of $0.08^{\prime \prime}$ pixel $^{-1}$. The short temporal cadence (around $3.4 \mathrm{sec}$ ) and short exposure time (0.1$1.6 \mathrm{sec}$ ) allows to study the evolution of short living and highly dynamic structures. The NFI can work in several modes that have a different observables and exposure time.

In our work (Chap.6), we use maps of the Stokes parameter I and V obtained by NFI in the Na I line $(5896 \AA)$ and over a range of $6 \AA$. These maps are recorded with a $0.16^{\prime \prime}$ pixel $^{-1}$ spatial scale with $2 \times 2$ pixel summing and field-of-view of $30.7^{\prime \prime} \times 81.9^{\prime \prime}$.

\subsubsection{Observation of the upper solar atmosphere (IRIS, SDO, Hi-C)}

\section{IRIS}

Presented here are the technical informations about IRIS, based on De Pontieu et al. (2014). The Interface Region Imaging Spectrograph (IRIS) is a space-based multi-channel imaging spectrograph. It was successfully launched on 27 June 2013 from Vandenberg Air Force Base in California. The main goal of the mission is to investigate the inter- 
face region (the chromosphere and the transition region). To this end, IRIS provides a spectrum together with images of the structures around the slit (slit-jaw image, SJI). IRIS is a Cassegrain telescope with a $19-\mathrm{cm}$ diameter of primary mirror and an effective focal length of $6.895 \mathrm{~m}$ and a $3^{\prime} \times 3^{\prime}$ field-of-view. IRIS is located at a Sun-Synchronous, low-Earth orbit (approximately 620-670 km above the Earth) with an inclination of 97.9 degrees. This orbit provides 8 months per year of solar observations without eclipses. The orbital period is 97.4 minutes.

\section{Spectroscopy with IRIS}

IRIS provides spectroscopic observations in two Far Ultra Violet channels (FUVS, FUVL) and one Near Ultra Violet band (NUV). The spectrum is obtained by the Czerny-Turner spectrograph and recorded by three CCD cameras, one for each channel. The FUV spectra have a spectral sampling of $12.8 \mathrm{~m} \AA$ and a spatial sampling of $0.167^{\prime \prime}$ pixel $^{-1}$ along the $0.33^{\prime \prime}$ pixel $^{-1}$ wide slit. The FUVS channel (Fig. 4.2), at wavelengths of 1332-1358 $\AA$, contain spectral lines of formation temperature at $\log T=3.7-7.0$, including two $\mathrm{C}_{\text {II }}(1335 \AA)$ lines. The FUVL channel (Fig. 4.1), at 1390-1406 ^ shows lines which correspond to the formation temperature $\log T=3.7-5.2$, including emission from $\mathrm{Si} \operatorname{IV}(1395 \AA)$ and $\mathrm{O}$ IV (1403 $\AA$ ). The NUV observation (Fig. 4.2), at 2785-2835 , are provided with $26 \mathrm{~m} \AA$ spectral resolution and the same spatial scale as FUV. This channel allows to study lines of formation temperature at $\log T=3.7-4.2$, including the $\mathrm{Mg}$ II k $(2796 \AA)$ and $\mathrm{Mg} \mathrm{II} \mathrm{h}$ (2803 $\AA$ ) lines and the wings of Mg lines. Maps with full spectral information are acquired through a raster procedure moving the solar image across the slit. The slit is nominally oriented parallel to the North-South axis of the Sun, but observations with a different orientation, in the range of -90 to +90 degrees are also possible. A large dense raster typically covers $140^{\prime \prime} \times 175^{\prime \prime}$ field-of-view. The typical cadence of spectroscopy observation is between 3-30 seconds. IRIS works in several modes, we briefly describe the most representative three of them. (1) In the fixed slit mode, the position of the slit is the same during the observation. (2) The sparse raster mode enables to observe the defined region-of-interest with a large raster step. (3) The dense raster mode provides observation with the highest spatial sampling (the smallest raster steps of $0.33^{\prime \prime}$ ).

In our analysis, we use only very large dense raster observation with a step size of $0.33^{\prime \prime}$ pixel $^{-1}$ and the field-of-view of $131.7^{\prime \prime} \times 175^{\prime \prime}$. 

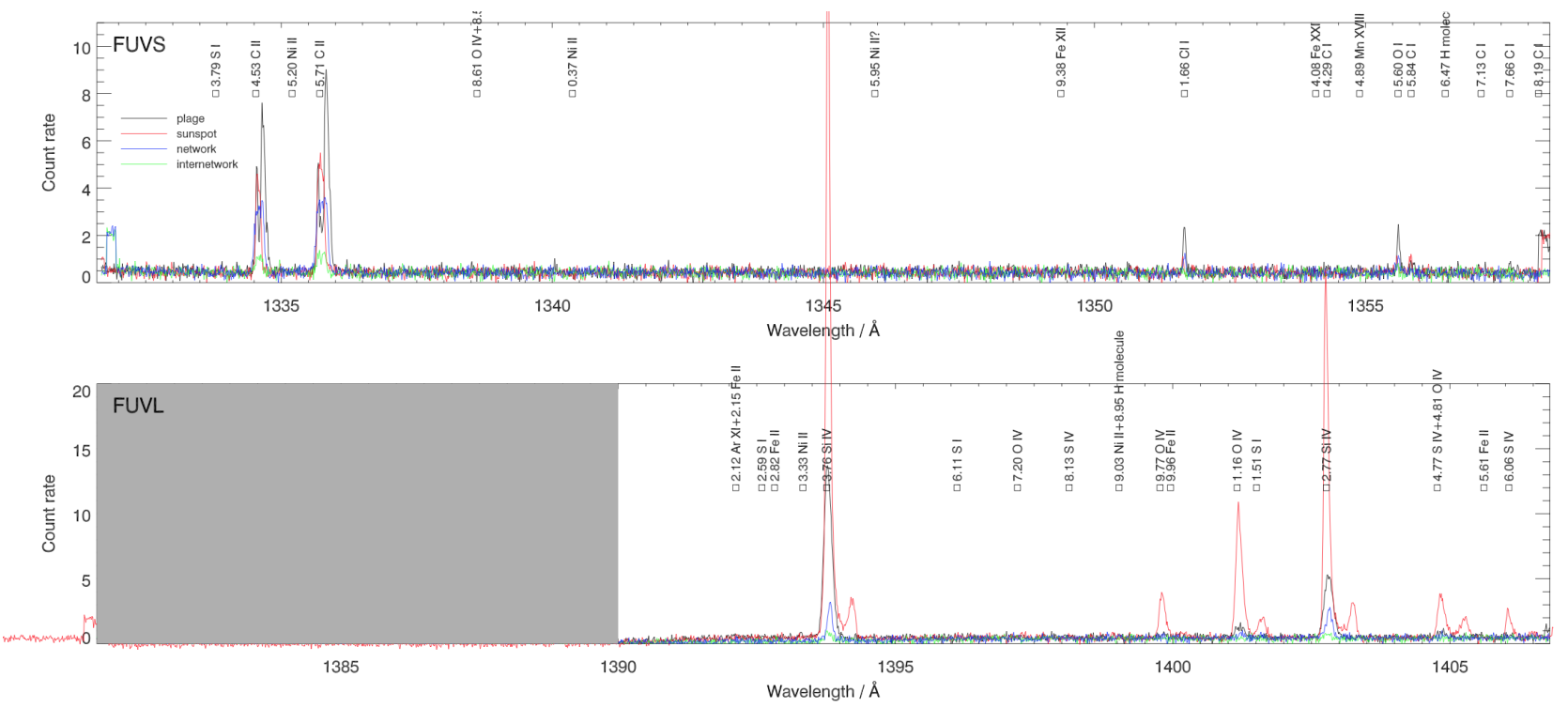

Figure 4.1: The FUV spectrum of IRIS for four different regions (Tian private communication).

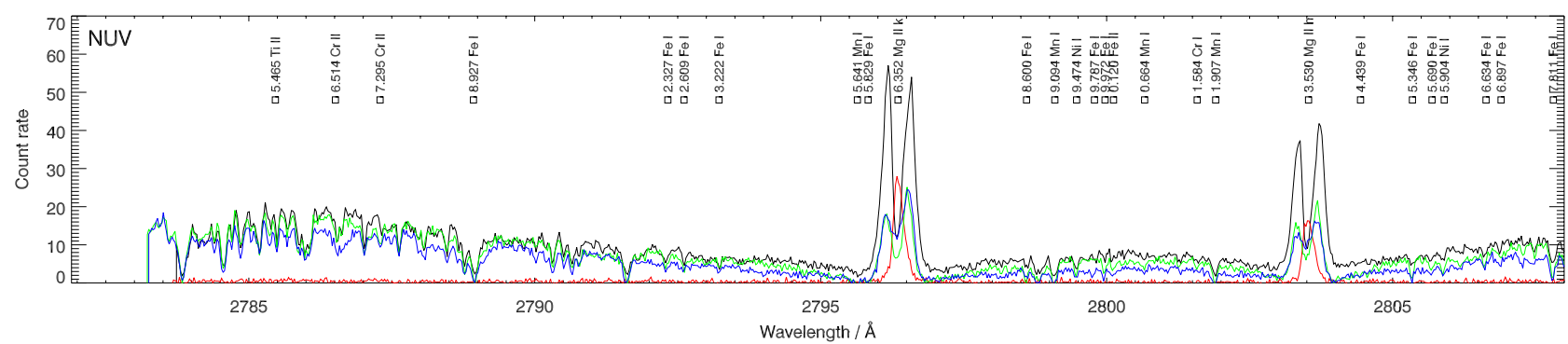

Figure 4.2: The NUV spectrum of IRIS for the same regions as in Fig.4.1(Tian private communication). 


\section{IRIS Slit-jaw-images}

The slit-jaw-image (SJI) camera observes the area surrounding the slit. The camera and a set of filters provides a field-of-view of $175^{\prime \prime} \times 175^{\prime \prime}$ with a spatial scale of $0.167^{\prime \prime}$ pixel $^{-1}$ at four different wavelength channels (Table 4.1). The strong lines such as $\mathrm{C}$ II, Si IV, $\mathrm{Mg}$ IIk and the wings of $\mathrm{Mg}$ II contribute most to the emission. However, since the passbands of the filters are broad, the emission from continuum and other lines can be significant. On each slit-jaw-images two fiducial marks on the slit are visible, which are used to align raster and slit-jaw images. The slit-jaw images are used in the analysis of the spatial and temporal evolution of structures in the solar atmosphere as well as to identify interesting regions for further spectroscopic studies.

Table 4.1: Details of IRIS slit-jaw channels (De Pontieu et al. 2014).

\begin{tabular}{|c|c|c|c|c|}
\hline Band-pass & Name & Center $[\AA]$ & Width $[\AA]$ & Temp. $[\log \mathrm{T}]$ \\
\hline$\overline{\mathrm{C}_{\text {II }}}$ & 1330 & 1340 & 55 & $3.7-7.0$ \\
\hline Si IV & 1400 & 1390 & 55 & $3.7-5.2$ \\
\hline Mg II h/k & 2796 & 2796 & 4 & $3.7-4.2$ \\
\hline Mg II wings & 2832 & 2830 & 4 & $3.7-3.8$ \\
\hline
\end{tabular}

\section{SDO/AIA}

The technical information about AIA onboard SDO presented here is based on Lemen et al. (2012). The Atmospheric Imaging Assembly(AIA; Lemen et al. 2012) is a multichannel imager which is used to investigate the structure and variability of the upper solar atmosphere.

Table 4.2: Details of SDO/AIA channels (Lemen et al. 2012).

\begin{tabular}{llll}
\hline \hline Channel & Primary ion(s) & Region of atmosphere & $\log$ T \\
\hline 4500 & continuum & photosphere & 3.7 \\
1700 & continuum & temperature minimum, photosphere & 3.7 \\
304 & He II & chromosphere, transition region & 4.7 \\
1600 & cont. + C IV ${ }^{⿴ 囗 十}$ & upper photosphere, transition region & $3.7,5.0$ \\
171 & Fe IX & quiet corona, upper transition region & 5.8 \\
193 & Fe XII, XXIV & corona and hot flare plasma & $6.2,7.3$ \\
211 & Fe XIV & active-region corona & 6.3 \\
335 & Fe XVI & active-region corona & 6.4 \\
94 & Fe XVIII & flaring corona & 6.8 \\
131 & Fe VIII, XXI & transition region, flaring corona & $5.6,7.0$ \\
\hline a In the 1600 channel dominant the continuum emission, the C Iv contribution is low \\
\multicolumn{3}{c}{ (almost none) in the quiet Sun and normal active region. }
\end{tabular}

The AIA is a system of four identical Cassegrain telescopes with an aperture of $20 \mathrm{~cm}$ and an effective focal length of $4.125 \mathrm{~m}$. The telescope is designed for observations in seven EUV channels, in range from 94-335 $\AA$, two UV channels, 1600 and $1700 \AA$, and a white light channel at $4500 \AA$ (Table 4.2 ). The AIA observations cover a temperature range from $6 \times 10^{4}$ to $2 \times 10^{7} \mathrm{~K}$ that corresponds to emission from the photosphere to the flaring corona. The CCD cameras, with $4096 \times 4096$ pixels, have a $40^{\prime} \times 40^{\prime}$ field-of-view 
which covers the full solar disk and an area up to 0.5 solar radii above the limb. Data are obtained with a plate scale of $0.6^{\prime \prime}$ pixel $^{-1}$ and with short exposure (0.5-3 s) and cadence (12 s). AIA provides almost continuous observations with high-precision pointing. Therefore, AIA data play an important role in studying the dynamics of the solar atmosphere, from short time-scale events to the large-scale solar variability at the time-scale of months, and more.

The main aim of AIA is to investigate the temporal and spatial evolution of structures as well as the thermal properties of the solar atmosphere.

\section{Hi-C}

The technical information, about Hi-C, presented here, based on Kobayashi et al. (2014). The High-resolution Coronal Imager (Hi-C; Cirtain et al. 2013), to investigate the solar corona, was launched on a sounding rocket on 11 July 2012 from White Sands Middile Range (USA) providing data for about 5 minutes. The main aim of the mission was to obtain images of an active region, with unprecedented spatial and temporal resolution, to study the dynamics of the small-scale structures.

Hi-C is a Ritchey-Chretien telescope with an aperture of $22.0 \mathrm{~cm}$, an effective focal length of $23.9 \mathrm{~m}$ and a focal ratio of $\mathrm{F} / 109$. It was designed to observe a $6.8^{\prime} \times 6.8^{\prime}$ fieldof-view with a plate scale of $0.103^{\prime \prime}$ pixel $^{-1}$ (average spatial resolution $0.3^{\prime \prime}$ ). The CCD camera with $4096 \times 4096$ pixels records images in one of two modes. In full detector mode $(4 \mathrm{k} \times 4 \mathrm{k})$, images are obtained with an exposure time of $2 \mathrm{~s}$ and a cadence of $5.4 \mathrm{~s}$. In $1 \mathrm{k} \times 1 \mathrm{k}$ mode, data are recorded only from the central part of the detector, with an exposure time of $0.5 \mathrm{~s}$ and a cadence of $1.4 \mathrm{~s}$. Hi-C observed the coronal emission from plasma at $1.5 \mathrm{MK}$ from Fe xII, in a passband of $5 \AA$ wide, centered around $193 \AA$. The Hi-C temperature response function is similar to SDO/AIA $193 \AA$.

The telescope observed active region AR11519-21, centred at [-130",-453"]. The observation in the full detection mode $(4 \mathrm{k} \times 4 \mathrm{k})$ started at 18:52:10 UT. During this phase the instrument was re-pointed at 18:54:34 UT. Hi-C obtained 36 high-resolution, fullframe images of the solar corona and 86 images with $1 \mathrm{k} \times 1 \mathrm{k}$ mode.

In this work, we use only $4 \mathrm{k} \times 4 \mathrm{k}$ data from the $\mathrm{Hi}-\mathrm{C}$ to analyse the geometrical properties of miniature-loops in the solar corona (Chap. 5).

\subsection{Data preparation (data levels)}

In this thesis mostly pre-processed data are used. Usually the raw data are named as level-0. Data with basic level of processing are called level-1. These data are typically corrected for bias, dark current and flat field. In this subsection, we briefly describe the pre-processing steps of Hi-C, AIA, HMI, Hinode and IRIS data.

We analyse Hi-C data of level-1.5 (mode $4 \mathrm{k} \times 4 \mathrm{k}$ ). They are corrected for dark, flatfield, dust and atmospheric absorption. These data are normalised for exposure time and are spatially co-aligned (Kobayashi et al.|2014).

We study level-1 data obtained by AIA and HMI onboard SDO. These images are corrected for dark, readout noise, flat-field, "bad" pixels and spikes. The images are transformed that the North direction is on the top (Lemen et al. 2012, Scherrer et al. 2012). The level-1 data are distributed by the Joint Science Operations Center (JSOC). The data 
level-1.5 can be produce from level-1 using the Solar Soft procedure aia_prep.pro ${ }^{1}$.

The processing of IRIS slit-jaw images is based on routines prepared for SDO and Hinode. In our study, we analyse level-2 data which are corrected for orientation, dark, offset, flat-field, "bad" pixels and spikes. The raster data of level-2 additionally have a preliminary wavelength calibration and geometrical alignment with the slit-jaw images (De Pontieu et al. 2014).

We use level-0 data obtained by Hinode/SOT which we reduce with the IDL routine fg_prep.pro ${ }^{2}$. It provides corrections for camera readout defects, dark current and spikes (Tsuneta et al. 2008).

\subsection{Data analysis technique}

\subsubsection{Differential emission measure (DEM)}

We analyse the inverse problem to determine the typical plasma temperature based on EUV observations. The energy flux $(F)$ of the optically-thin medium is proportional to the total emissivity ( $\varepsilon$, eq. 3.15) along line of sight $(h)$ :

$$
F=\int \varepsilon d h
$$

Substitute eq. 3.15 into eq. 4.1 to obtain:

$$
F=\int_{h=0}^{\infty} G(T) n_{e}^{2} d h
$$

The solar atmosphere is highly dynamics, therefore usually the scale of the temperature $(T)$ is used, so eq. 4.2 is rewritten as:

$$
F=\int G(T) \underbrace{n_{e}^{2}\left(\frac{d T}{d h}\right)^{-1}}_{:=D E M} d T,
$$

where $\operatorname{DEM}(\mathrm{T})$ is the Differential Emission Measure $\left[\mathrm{cm}^{-5} \mathrm{~K}^{-1}\right]$. It provides information of the relative contributions from plasma at different temperature (Aschwanden 2004). Thus, for an emission line $i$ the energy flux is:

$$
F_{i}^{(D E M)}=\int_{T} G_{i}(T) D E M(T) d T .
$$

The contribution function $\left(G_{i}\right)$ contains the atomic physics parameters. It can be computed based on the CHIANTI database.

The above equation is solved as an inverse. Starting with the guest solution for the DEM the $F_{i}^{(D E M)}$ is tweaked such the parameters:

\footnotetext{
${ }^{1}$ The IDL routine iris_prep.pro is provided by Solar Soft: (https://darts.isas.jaxa.jp/pub/ ssw/iris/idl/lmsal/calibration/iris_prep.pro).

${ }^{2}$ The IDL routine fg_prep.pro is provided by Solar Soft: (http://darts.jaxa.jp/pub/solar/ssw/ hinode/sot/idl/fg/cal/fg_prep.pro).
} 


$$
\min \left(\sum_{i}\left|F_{i}^{(D E M)}-F_{i}^{(o b s)}\right|^{2}\right)
$$

is minimum, where the $F_{i}^{(o b s)}$ is the energy flux obtained from observation.

In this thesis, the energy flux obtained from SDO/AIA observation is used. It depends of the instrumental response function $\left(\alpha_{i}\right)$, the contribution function and the differential emission measure:

$$
F^{A I A}=\sum_{i} \int_{T} \alpha_{i} G_{i}(T) \operatorname{DEM}(T) d T=\int_{T} \underbrace{\left(\sum_{i} \alpha_{i} G_{i}(T)\right)}_{:=f(T)} \operatorname{DEM}(T) d T,
$$

where $f(T)$ is the temperature response function. The temperature response function is calculated for each AIA channel separately. The curves of the temperature response functions are presented in Fig. 4.3 .

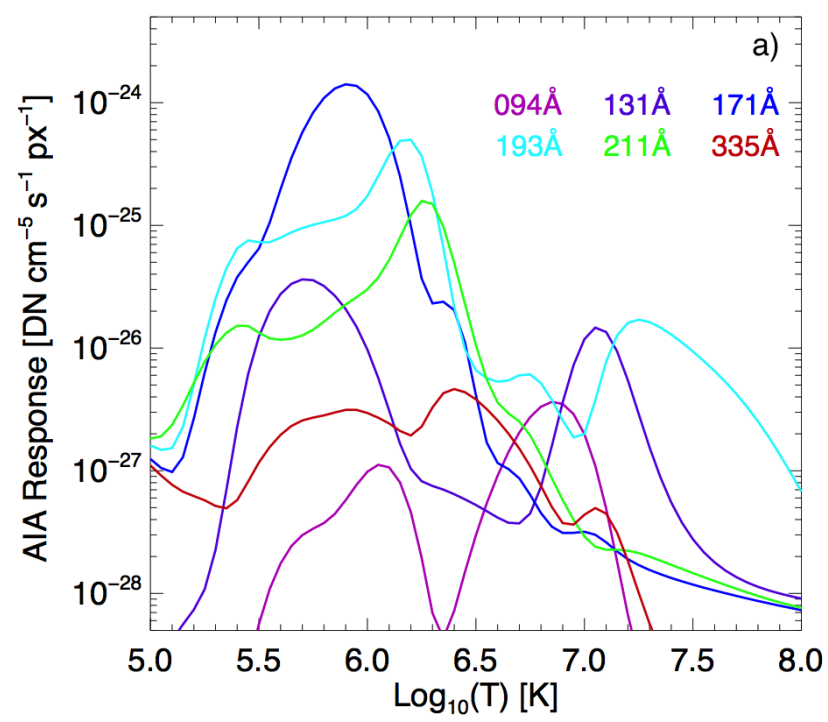

Figure 4.3: The temperature response functions of six SDO/AIA channels (Lemen et al. (2012).

The DEM method is limited, it describes the plasma properties only along the line of sight. One can find a small fraction high-density plasma at low temperature and a large fraction of a low-density plasma at the high temperature which can give the same DEM. Distinguishing between the two cases is possible only using additional data (Mariska 1992). In this work, the DEM is computed with the method presented by Hannah and Kontar (2012).

\subsubsection{Correlation coefficients}

A correlation coefficient is a measure of the statistical relationship between two or more random variables. The direction and strength of the correlation are described by the sign 
and absolute value of correlation coefficient, respectively. The correlation coefficient has values in a range between -1 (perfect anticorrelation) and 1 (perfect correlation), while 0 describe a lack of correlation (uncorrelated variable). In the case of two variables, the correlation is positive, when changes of both variables characterise the same trends. For the opposite trends, the correlation is negative (anticorrelation). If the absolute value of the correlation coefficient is closer to one, this indicates a stronger dependence between the variables. When the correlation coefficient is near zero, then the association is weak.

There are many types of correlation coefficients, such as, Pearson's linear correlation coefficient which measure a linear association, Spearman's order rank correlation coefficient describing the non-linear relationship, the cross-correlation is used to analyse association between 2D arrays (images). Here, we present a brief overview of correlation coefficients that are used in this thesis.

\section{Pearson's correlation coefficient}

The Pearson's correlation coefficient measures a linear relationship between two variables $(x$ and $y$ ). We follow Press et al. (2002) in assumming the bivariate normal distribution of variables; $x$ and $y$ are stochastically independent; and the relation between them is linear $(y=a x+b)$, then the linear correlation coefficient $(r)$ is:

$$
r \equiv \frac{\sum_{i}^{n}\left(x_{i}-\bar{x}\right)\left(y_{i}-\bar{y}\right)}{\sqrt{\sum_{i}^{n}\left(x_{i}-\bar{x}\right)^{2}} \sqrt{\sum_{i}^{n}\left(y_{i}-\bar{y}\right)^{2}}}
$$

The pair of measurements are marked as $\left(x_{i}, y_{i}\right)$, where $i=(0,1,2 \ldots, n)$ defines the number of measurements and $\bar{x}, \bar{y}$ are mean of $\mathrm{x}$ and $\mathrm{y}$, respectively.

The Pearson's correlation coefficient is most often used in linear relationship studies. In Fig. 4.4, we present examples of scatter plots for different relationships between two variables and calculated their correlation coefficients. The scaling and order of the data do not have an influence for the correlation coefficient, however, it is sensitive to outliers.

For a more detailed description of Pearson's correlation coefficient we refer to Press et al. (2002). The calculation of the Pearson linear correlation coefficient is provided by the CORRELATE $\mathrm{E}^{3}$ function in IDL (from version 4.0).

\section{Cross-correlation coefficient}

The cross-correlation coefficient is used to identify similar patterns in image analysis. This coefficient describes the relationship between intensity pattern of two or more images. If $\mathrm{M}$ and $\mathrm{N}$ are two-dimmensional arrays of intensities with the same sizes $(i \times j)$, then the cross-correlation coefficient is defined as:

$$
r \equiv \frac{\sum_{i} \sum_{j}\left(M_{i j}-\bar{M}\right)\left(N_{i j}-\bar{N}\right)}{\sqrt{\sum_{i} \sum_{j}\left(M_{i j}-\bar{M}\right)^{2}} \sqrt{\sum_{i} \sum_{j}\left(N_{i j}-\bar{N}\right)^{2}}}
$$

The images in Fig. 4.7 demonstrate the usage of the cross-correlation coefficient to describe the relationship between the original image and images contaminated by noise.

In our study, we use the IDL function C_CORRELATE ${ }^{4}$ to calculate the cross-correlation

${ }^{3}$ CORRELATE: https://www .harrisgeospatial. com/docs/CORRELATE.html

${ }^{4}$ C_CORRELATE: https://www.harrisgeospatial.com/docs/C_CORRELATE.html 

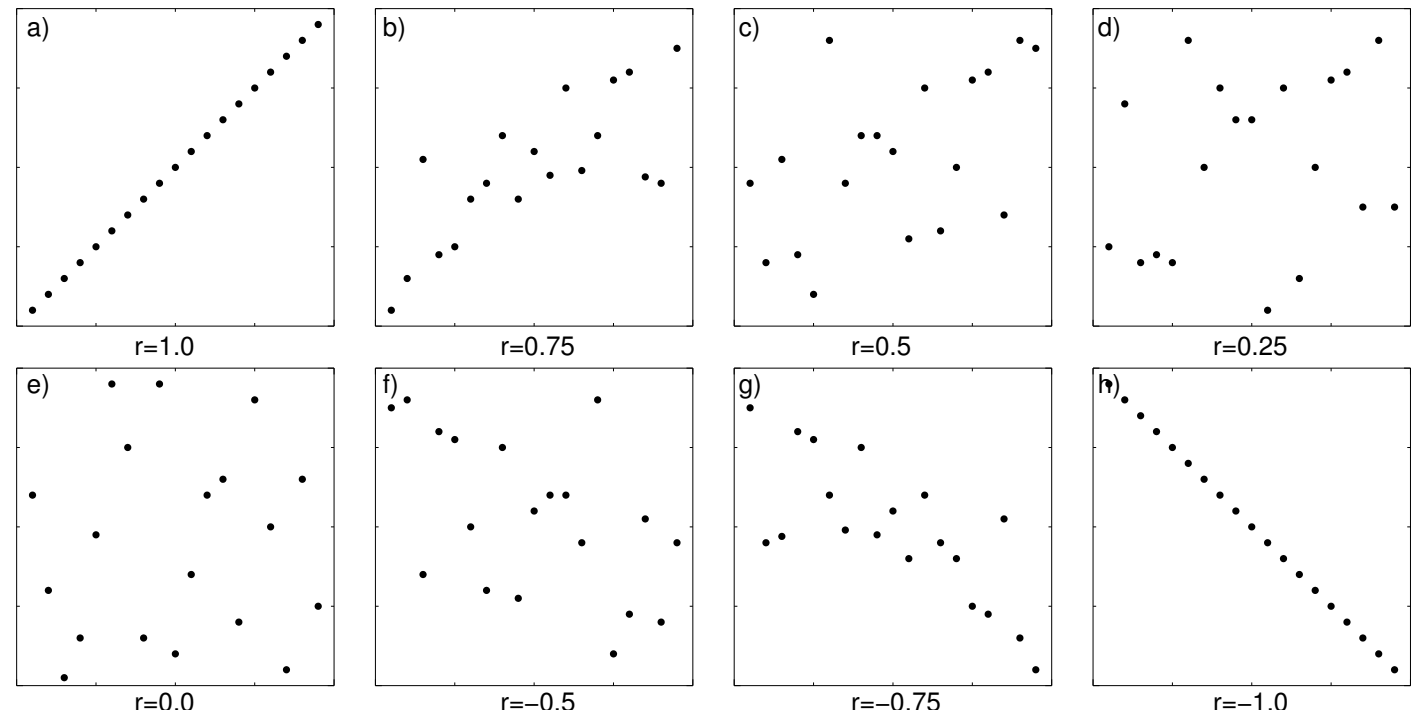

Figure 4.4: Correlation scatter plots and Pearson's correlation coefficients. In the case of perfect correlation (a), all points are located on the straight line and both variables increasing. The correlation coefficient falls out with increasing scattering of the points $(b$, c). The $r=0$ indicates the lack of dependence between variables (e). When two variables follow an opposite trend then the correlation coefficient is negative ( $\mathrm{f}, \mathrm{g}, \mathrm{h}$ ). In perfect anti-correlation (h), all points are exactly on the straight line and $r=-1$.

coefficient. For more details about the cross-correlation we refer to Fuller (1995).

\section{Spearman correlation coefficent}

The Spearman's rank order correlation coefficient is a test of a non-linear association between two variables ( $x$ and $y$ ). It is used for a monotonic (not necessarily linear) relation between variables, for any distribution.

The algorithm for computing this correlation is presented by Press et al. (2002). In this method, pairs of $\left(x_{i}, y_{i}\right)$ are grouped in the rank and for each of them the mean value is calculated. The Spearman correlation is computed as a linear correlation of ranks.

The Spearman's correlation coefficient is insensitive to outliers. Therefore it is used to analyse a highly scattered or poor quality data. It can be used also to study the linear relationship between variables instead of the Pearson's correlation coefficient, when the assumption of the normal distribution is not applicable or when the number of outliers is significant. However, the Pearson's correlation is a quantity measure of an association, whereas the Spearman's correlation coefficient is a quality measure. The Spearman's correlation is symmetric due to changes of variables.

In Fig. 4.6, we present the usage of the Spearman's correlation coefficient. The IDL function R_CORRELATE ${ }^{5}$ allows to calculate the Spearman's correlation coefficient.

\footnotetext{
${ }^{5}$ R_CORRELATE: https://www .harrisgeospatial.com/docs/R_CORRELATE.html
} 


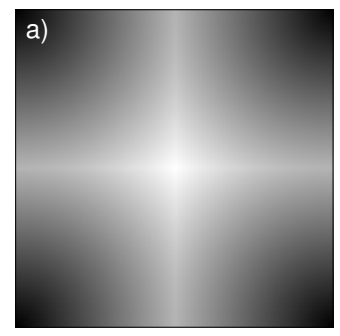

oryginal $(r=1.0)$

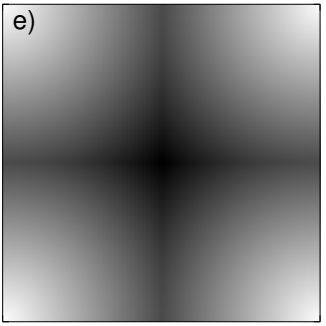

$r=-1.0$

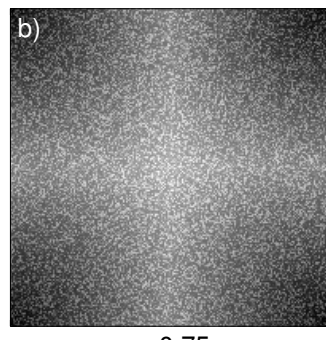

$r=0.75$

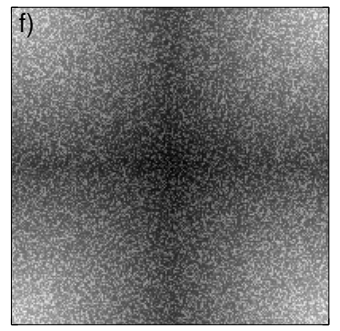

$r=-0.75$

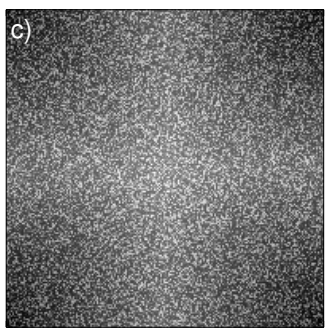

$r=0.5$

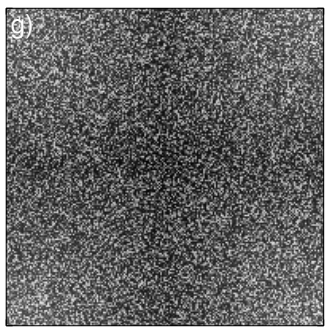

$r=-0.25$

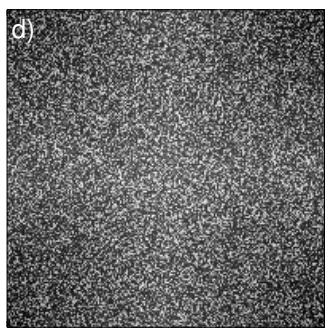

$r=0.25$

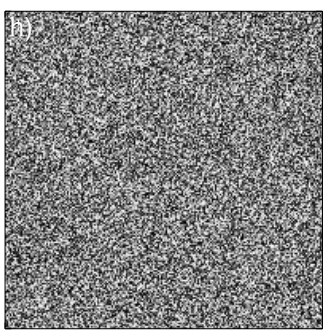

$r=0.0$

Figure 4.5: A series of images with added different noise level and the cross-correlation coefficients. The cross-correlation coefficient is calculated between image without noise (a) and rest of images. The correlation of image without noise with itself (a) gives $r=1$, perfect correlation. The increasing noise level (differences between images) causes decrease of the correlation coefficient $(b, c, d)$. These analysis are provided to the complement image (e) and complement images with noise (f, g). The complement images (e, f, g) are anti-correlate with original one (a). The two images without common patterns (h) have $r=0$.
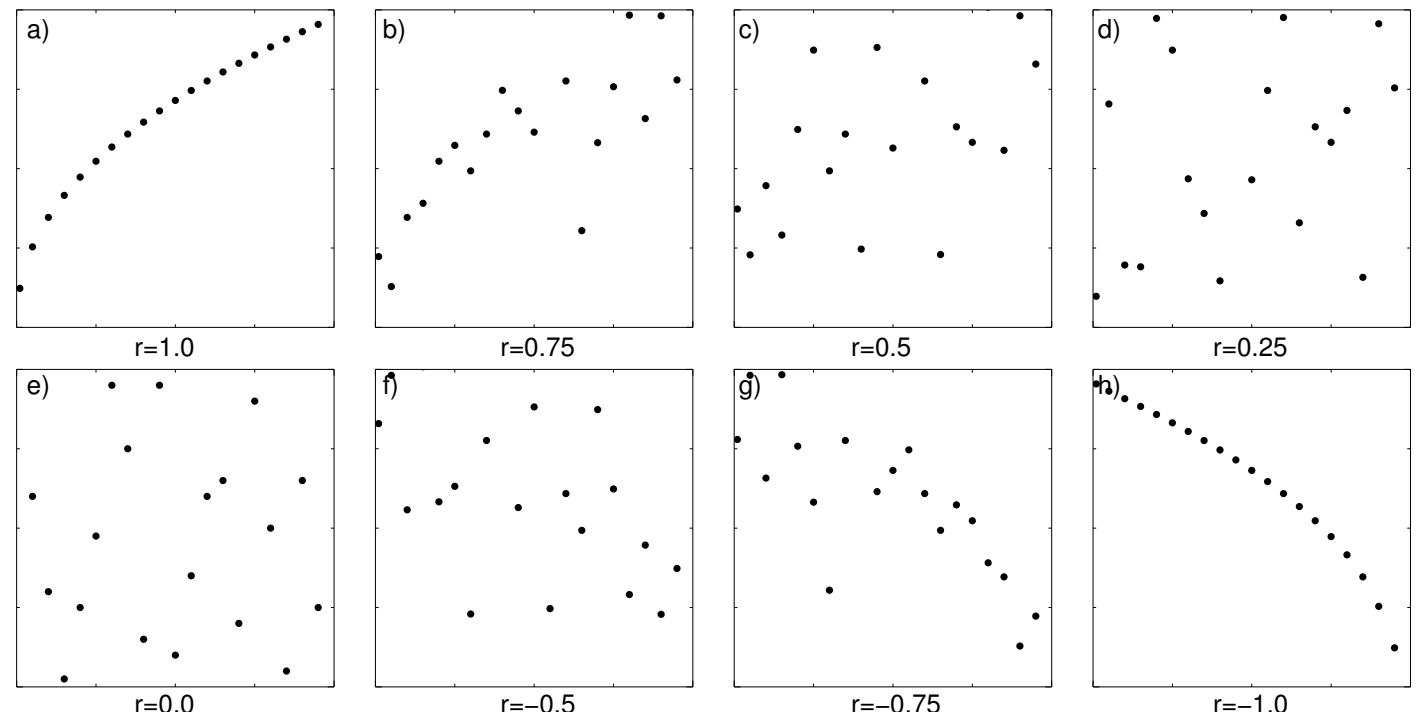

Figure 4.6: Scatter plots with non-linear correlation and Spearman's correlation coefficients. A perfect correlation (a), $r=1$, is for a positive monotonic relationship between two variables (not necessarily a linear relationship). The correlation coefficient decreases with increasing scattering of the points (b, c, d). For $r=0$ (e), the variables are uncorrelated. The negative correlation coefficient indicates anti-correlation ( $f, g, h$ ). 


\subsubsection{Co-aligning}

High precision aligment is important to compare images which are obtained with different instruments, especially if the target of the analysis are small-scale structures. From the technical point of view, the aim of the co-aligning is to define a shift between two images $(A$ and $B$ ) for which the cross-correlation coefficient has a maximum value, as clearly shown in Fig.4.7, In this thesis, we use two co-aligning methods.

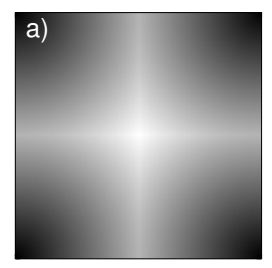

oryginal

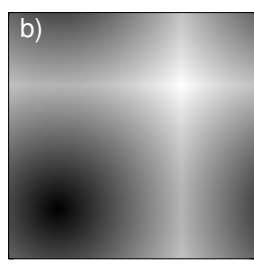

$r=0.28$

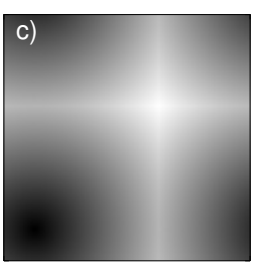

$r=0.68$

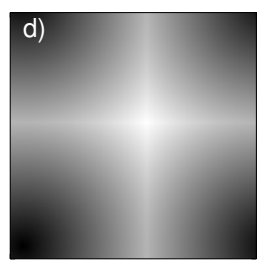

$r=0.94$

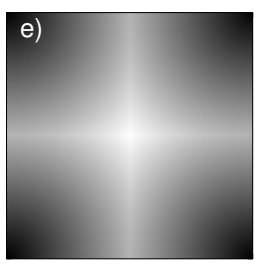

$r=1.0$

Figure 4.7: A series of shifted images and the cross-correlation coefficient. The original $100 \times 100$ pixel image (a) is shifted by 40 (b), 25 (c), 10 (d) and 0 (e) pixels in $x$ and $y$ directions. The cross-correlation coefficient calculated between the original image and the shifted images decreases with increasing shift.

The procedure align . pro ${ }^{6}$ is an IDL routine to compute the cross-correlation coefficient based on the FFT technique. We use this method to align images with clear large scale structures and in a preliminary co-aliment of large image sequece. This method provides a sub-pixel accuracy of aligning.

The second routine that we use to image co-alignment is rigidalign $1 . p r o 7^{7}$. In this method, a user defines a shift range. Then the image $B$ is shifted respect to image $A$ in this range with a one-pixel step, in $x$ - and $y$ - directions. For each step, the cross-correlation coefficient is calculated. Finally, the algorithm searches for the maximum of the crosscorrelation coefficient. The shift is calculated taking into account the interpolated crosscorrelation coefficients for the maximum value and surroundings. We use this routine to align the images with a sub-pixel accuracy precision, especially to align the low-contrast images.

The aling . pro routine is significantly faster than the rigidalign1.pro. However, the second one works better with low-contrast images, therefore we use it to align images of the quiet Sun or coronal hole. Both methods can be used only to calculate a translation. More advanced algorithms are necessary to correct for rotation and stretching. In this thesis, we use alignment routines only to find a proper transposition. In some cases, we apply a small correction for a rotation, but it based on the header information.

\footnotetext{
${ }^{6}$ The image co-aligning routine align.pro was written by Roberto Luis Molowny Horas: (http://folk. uio.no/eamonms/crispex/lapalma/spreduc/fit/align.pro).

${ }^{\bar{T}}$ The routine rigidalign1.pro was written by Lakshmi Pradeep Chitta (private communication).
} 


\section{Miniature loops in the solar corona}

*This chapter is published as a journal article "Miniature loops in the solar corona" (DOI 10.1051/0004-6361/201629247) in Astronomy \& Astrophysics. Credit: Barczynski, K.; Peter, H.; Savage, S. L., A\&A, 599, A137, (2017), reproduced with permission (C) ESO.

Context: Magnetic loops filled with hot plasma are the main building blocks of the solar corona. Usually they have lengths of the order of the barometric scale height in the corona that is $50 \mathrm{Mm}$.

Aims: Previously it has been suggested that miniature versions of hot loops exist. These would have lengths of only $1 \mathrm{Mm}$ barely protruding from the chromosphere and spanning across just one granule in the photosphere. Such short loops are well established at transition region temperatures $(0.1 \mathrm{MK})$, and we investigate if such miniature loops also exist at coronal temperatures $(>1 \mathrm{MK})$.

Methods: We used extreme UV imaging (EUV) observations from the High-resolution Coronal Imager (Hi-C) at an unprecedented spatial resolution of $0.3^{\prime \prime}$ to $0.4^{\prime \prime}$. Together with EUV imaging and magnetogram data from the Solar Dynamics Observatory (SDO) and X-Ray Telescope (XRT) data from Hinode we investigated the spatial, temporal and thermal evolution of small loop-like structures in the solar corona above a plage region close to an active region and compared this to a moss area within the active region.

Results: We find that the size, motion and temporal evolution of the loop-like features are consistent with photospheric motions, suggesting a close connection to the photospheric magnetic field. Aligned magnetograms show that one of their endpoints is rooted at a magnetic concentration. Their thermal structure, as revealed together with the X-ray observations, shows significant differences to moss-like features.

Conclusions: Considering different scenarios, these features are most probably miniature versions of hot loops rooted at magnetic concentrations at opposite sides of a granule in small emerging magnetic loops (or flux tubes). 


\subsection{Introduction}

The major building blocks of the solar corona are loops. Observations of these structures have existed since the 1940s (Bray et al.|1991), with the key information acquired through extreme UV (EUV) and X-ray observations. Coronal loops cover a wide range of temperatures and lengths; from small transition region loops at $0.1 \mathrm{MK}$ being only a few $\mathrm{Mm}$ long (Peter 2001, Hansteen et al. 2014) to loops hotter than $10 \mathrm{MK}$ and/or longer than $100 \mathrm{Mm}$ (Reale 2010). A typical active region loop would have a temperature of approximately $3 \mathrm{MK}$ and a length above $10 \mathrm{Mm}$ (Reale 2010). Naturally, the question appears regarding the possible minimum length of a hot ( $>1 \mathrm{MK})$ coronal loop. Magnetic field lines originating from very small bipoles might not reach above the height of the average chromosphere, which is, according to semi-empirical models, at some $2 \mathrm{Mm}$. Assuming a semi-circular geometry of the field line, this would correspond to a footpoint distance of $4 \mathrm{Mm}$ in the photosphere. However, the solar atmosphere is in a dynamic state, therefore one might expect loops even shorter than that.

The presence small bipolar magnetic structures carried upward either by granular convection or magnetic buoyancy was proposed by Lites et al. (1996) in the context of horizontal inter-network magnetic fields. Using spectro-polarimetry Centeno et al. (2007) showed that such low-lying magnetic loops can connect opposite magnetic polarities that are separated by only $2^{\prime \prime}$ in the photosphere. Such small magnetic bipoles can emerge, isolated in a transient fashion (Ishikawa et al. 2008) with a mean lifetime of approximately 4 minutes (Ishikawa and Tsuneta 2009). In small-scale emergence processes the separation between the footpoints in the photosphere ranges from $0.5 \mathrm{Mm}$ to $4 \mathrm{Mm}$ and is correlated with the lifetime of the emerging structure (Martínez González and Bellot Rubio 2009). To reconstruct the three-dimensional structure of the magnetic field during the small-scale mergence, Ishikawa et al. (2010) investigated spectro-polarimetric data to invert the magnetic field vector as a function of height in the atmosphere. They showed that the rising structures are indeed flux tubes with enhanced magnetic field reaching heights of $400 \mathrm{~km}$ above optical depth unity at the surface (their Fig. 8). Their diagnostics were limited to the photosphere, but it seems reasonable to assume that such a flux tube could rise all the way to the top of the chromosphere if the emerging field was strong enough.

Indirect evidence for the existence of small cool transition region loops was first suggested by Feldman (1983) based on spectroscopic data. In particular, he argued that part of the transition region emission originates in unresolved fine structures, which would be cool loops not connected to the corona above. With the help of spectral maps and spectroscopic investigations Warren and Winebarger (2000) and Peter (2000) investigated the properties of such transition region loops. Because of instrumental limitations, these could not be imaged directly in a clear fashion until the IRIS spectrograph and imager (De Pontieu et al. 2014) became available. Using slit-jaw images, Hansteen et al. (2014) could follow the evolution of such cool loops with lengths of only a few Mm and lifetimes of a few minutes. Appearing in the quiet Sun network, considering their length, such loops would only span across one granule, probably connecting opposite magnetic polarities in the inter-granular lanes. Such small cool transition region loops have also been investigated in one-dimensional models. Sasso et al.(2012) showed that loops with lengths of some $1 \mathrm{Mm}$ to $15 \mathrm{Mm}$ could in principle explain the increase of the emission measure towards lower temperatures below $10^{5} \mathrm{~K}$. In their models the quasi-static loops 


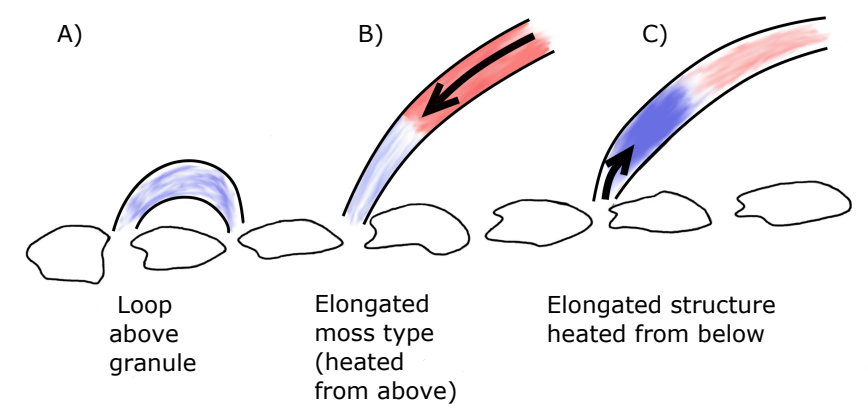

Figure 5.1: Three scenarios for miniature loop-like structures seen between $1 \mathrm{MK}$ and 2 MK. (a) Small loops spanning across one granule, (b) short elongated structures at the footpoint of a hot loop, e.g. moss, and (c) footpoint segments of a dilute hot loop heated from below (see Sect.5.1). The black solid lines indicate granules. The blue and red colours in the loop features denote warm (1 MK to $2 \mathrm{MK}$ ) and hot ( $>5 \mathrm{MK}$ ) plasma. The arrows represent the direction of the energy flux powering the bright feature, that is, in panel (b) the heat flux from the corona down to the lower atmosphere and in panel (c) the Poynting flux into the upper atmosphere.

always remained well below $1 \mathrm{MK}$.

With the existence of small cool loops now being firmly established, the question is; can such small loops also reach higher coronal temperatures? One conceptual argument against this would be that a short magnetic fieldline with a length of only one or a few $\mathrm{Mm}$ would still be covered by the chromosphere. Because of the high density there, it might be unlikely in terms of energy requirements to heat a significant amount of material to coronal temperatures. However, recent spectroscopic observations have shown evidence of plasma in the dense transition from the photosphere to the chromosphere at approximately the temperature minimum perhaps being heated to almost $100000 \mathrm{~K}$ (Peter et al.2014). Therefore, it might well be that such structures are further heated to coronal temperatures.

Small elongated structures with a footpoint distance of only $1 \mathrm{Mm}$ reaching more than $1 \mathrm{MK}$ have been reported by Peter et al. (2013). Using data obtained from the High Resolution Coronal Imager (Hi-C; Cirtain et al. 2013), they found these structures to have a width of less than $200 \mathrm{~km}$. These observations were only possible because of the high spatial resolution of the suborbital rocket experiment Hi-C. Its resolution is approximately five times higher than that of the current workhorse of coronal imaging studies, the Atmospheric Imaging Assembly (AIA; Lemen et al. 2012), which has a spatial resolution of approximately $1.4^{\prime \prime}$ corresponding to $1 \mathrm{Mm}$ and would not show those extremely small features.

The small-scale coronal structures have been interpreted as miniature loops by Peter et al. (2013), spanning one granule (Fig. 5.19) and connecting two opposite magnetic field polarities in the inter-granular lanes. This would provide a natural explanation for their length, which is comparable to a granule, and be consistent with the emerging bipoles discussed above. Also, the motion of such miniature loops should be of the order of the photospheric horizontal motions which is typically of the order of $1 \mathrm{~km} \mathrm{~s}^{-1}$ (e.g. Dravins 1975). However, Peter et al. (2013) could not exclude an alternative scenario in which 
these elongated structures would be the moss-type emission (Fig.5.1p). Moss emission is characterised by dynamic arcsecond-scale features at the footpoint region of a hot loop typically reaching more than $5 \mathrm{MK}$ and being visible in X-rays (Berger et al. 1999). In some sense the $1 \mathrm{MK}$ to $2 \mathrm{MK}$ emission near the footpoints represents the transition region of that hot loop. In the HiC data set Testa et al. (2013) and Morton and McLaughlin (2014) analysed moss structures but in the more active part of the HiC field-of-view, and not in the plage region where Peter et al. (2013) found the small elongated structures. There would also be a third option, where a longer structure is heated from below, filling only the lower part of the loop with $1 \mathrm{MK}$ to $2 \mathrm{MK}$ plasma near the footpoint with a dilute (basically invisible) hot part above (Fig. 5.1\%).

The small structures we report here are quite different from coronal bright points, originally observed by Golub et al. (1974) in X-rays. Those are much bigger with an overall average size of approximately $30^{\prime \prime}$ and a bright core of approximately $5^{\prime \prime}$ to $10^{\prime \prime}$. They have much longer lifetimes of several hours and are typically associated with a bipolar magnetic feature at the surface. However, there is a (magnetic) substructure in these features (Brown et al. 2001), and it has been suggested that a bright point might consist of small loops with widths of only approximately $1^{\prime \prime}$ to $2^{\prime \prime}$ and temperatures of approximately 1.6 MK (Dere 2008). Nevertheless, it does not seem likely that the tiny loop-like features directly observed by Peter et al. (2013) and investigated here are related to the proposed elementary structures of a coronal bright point. This is supported by the magnetic structure, which is clearly bi-polar for a bright point, but mostly unipolar (perhaps with small-scale non-resolved opposite polarities) for the plage-type region hosting the very small loop-like features.

Our aim is to understand the nature of the tiny elongated structures identified by Peter et al. (2013) and to distinguish between the three scenarios outlined above and sketched in Fig. 5.1. In particular, we investigate the morphology and evolution (Sect. 5.3), the underlying magnetic field (Sect.5.4), and the thermal properties (Sect. 5.5) of these features.

\subsection{Observations}

In this study we concentrated on data acquired during a sub-orbital sounding rocket flight of the High-resolution Coronal Imager (Hi-C; Cirtain et al. 2013, Kobayashi et al. 2014). It was launched on 11th July, 2012, and acquired data for approximately 5 minutes of the active region AR11519-21 and its surroundings. For our analysis, we complement this (Table 5.1) with data from the X-Ray Telescope onboard Hinode (XRT; Golub et al. 2007) and the Solar Dynamics Observatory (SDO); in particular the extreme UV images from the Atmospheric Imaging Assembly (AIA; Lemen et al. 2012) and magnetograms from the Helioseismic and Magnetic Imager (HMI; Scherrer et al. 2012).

The extreme UV imager Hi-C provides data of the solar corona in a $5 \AA$ wide wavelength band around $193 \AA$ dominated by emission from Fe XI originating at approximately 1.5 MK. In the context of coronal imaging, $\mathrm{HiC}$ has an unprecedented plate scale of $0.1^{\prime \prime}$ pixel $^{-1}$, which corresponds to $73 \mathrm{~km} \mathrm{pixel}^{-1}$ on the Sun. The image resolution estimated by Winebarger et al. (2014) is $0.3^{\prime \prime}$ to $0.4^{\prime \prime}$. The $4 \mathrm{k} \times 4 \mathrm{k}$ full-frame images were recorded with an exposure time of $2 \mathrm{~s}$ at a cadence of $5.5 \mathrm{~s}$. The unprecedented spatial and temporal resolution achieved by the Hi-C allows us to carry out the detailed analysis of 
Table 5.1: Imaging data used in this study.

\begin{tabular}{|c|c|c|c|}
\hline & band & contribution & $\overline{T_{\text {peak }}[\mathrm{MK}]^{\mathrm{c}}}$ \\
\hline $\mathrm{Hi}-\mathrm{C}$ & $193 \AA$ & Fe XII & 1.5 \\
\hline \multirow{7}{*}{$\mathrm{AIA}^{\mathrm{a}}$} & $1600 \AA$ & continuum & $<0.01$ \\
\hline & $131 \AA$ & $\mathrm{Fe}$ VIII & 0.4 \\
\hline & $171 \AA$ & Fe IX & 0.7 \\
\hline & $193 \AA$ & Fe XII & 1.5 \\
\hline & $211 \AA$ & Fe XIV & 2.0 \\
\hline & $335 \AA$ & Fe XVI & 2.8 \\
\hline & $94 \AA$ & Fe XVIII & 7.1 \\
\hline $\mathrm{XRT}^{\mathrm{b}}$ & Ti-Poly & free-free emission & 9 \\
\hline \multicolumn{4}{|c|}{$\begin{array}{l}\text { a All listed AIA channels are used to co-align the Hi-C } \\
\text { observations with the HMI magnetograms, and all but } \\
\text { the } 1600 \AA \text { channel are employed in the DEM analysis } \\
\text { (Sect.5.5.1). } \\
\text { b } \\
\text { c The XRT observations are used for thermal diagnostics. } \\
\text { For the HiC and AIA bands, } T_{\text {peak }} \text { is the temperature of } \\
\text { peak ion fraction of the main contributing ion in the re- } \\
\text { spective band according to O'Dwyer et al. (2010). Most } \\
\text { AIA bands have significant contributions from other } \\
\text { temperatures as well. The value for XRT is taken from } \\
\text { Golub et al. (2007). }\end{array}$} \\
\hline
\end{tabular}

the small-scale arcsec-size structures discussed in the introduction that were not resolved by previous instruments. We have studied 25 full-frame images (level-1.5) of the active region obtained between 18:53:11 UT and 18:55:30 UT on 11th July, 2012. All times in this paper are in seconds from this first frame. We do not analyse the earlier 11 images (before 18:53:11 UT), because at this point the focus was not yet locked. The images after 18:55:30 contain only a partial read out of the detector $(1 \mathrm{k} \times 1 \mathrm{k})$ acquired at a higher cadence, which unfortunately did not cover the region we are interested in here. The Hi$\mathrm{C}$ images are aligned to sub-pixel level accuracy using a cross-correlation technique to obtain a jitter-free data set.

For context, for thermal studies (Sect.5.5.1), and the alignment with the magnetic field data (Appendix 5.8.2), we used data from various channels of AIA (cf. Table 5.1). This provided full-disk images at a plate scale of $0.6^{\prime \prime}$ pixel $^{-1}$ at a cadence of $12 \mathrm{~s}$ or more. In Fig. 5.2 we show the context of the active region observed by Hi-C as well as our regions of interest. In this paper we concentrate on two small plage-type regions that are shown in Fig. 5.2 $\beta$ and $\gamma$ along with areas marked for a more detailed study.

To investigate the relationship between the small loop-like features and the surface magnetic field, we use magnetogram data from HMI. Provided with a plate scale of $0.5^{\prime \prime}$ pixel $^{-1}$ and a temporal cadence of $45 \mathrm{~s}$, HMI provides only a few snapshots during the $140 \mathrm{~s}$ of the full-frame Hi-C data used here. Thus, we concentrate on the line-ofsight magnetogram taken at 18:54:53 UT in the middle of the Hi-C full-frame coverage. The spatial alignment between HMI and $\mathrm{Hi}-\mathrm{C}$ was achieved through a sequence of AIA channels using a cross-correlation procedure (see Appendix 5.8.2). We estimate the accuracy of this alignment to be better than $0.2^{\prime \prime}$ (Table 5.2), that is, slightly more than half 

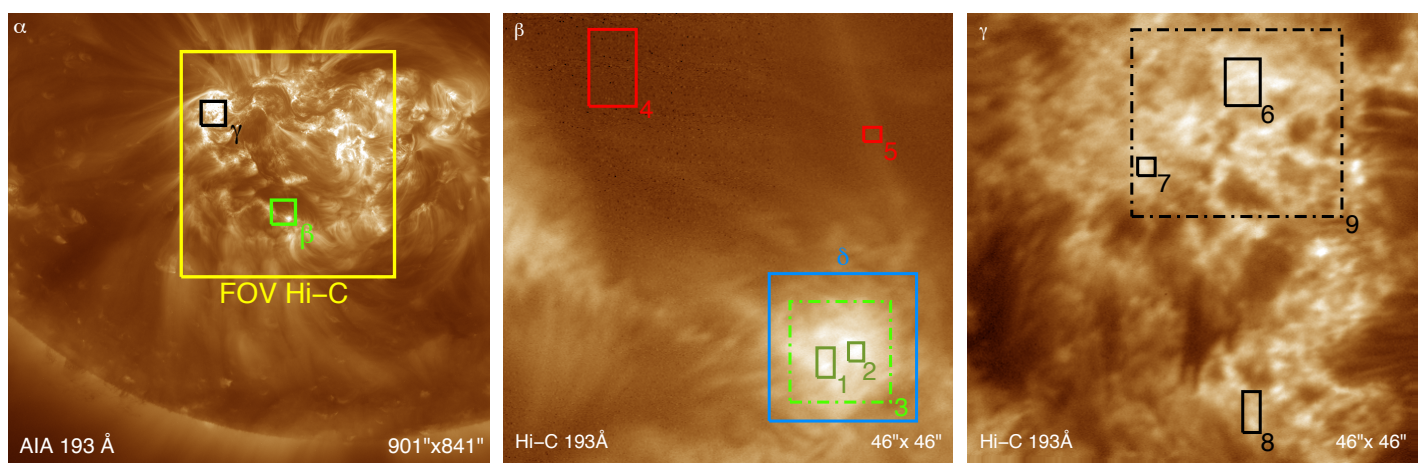

Figure 5.2: Active region context and regions of interest. All images are taken in the $193 \AA$ A channel showing emission around 1.5 MK (see Table 5.1). Panel ( $\alpha$ ) shows part of the solar disk seen in AIA during the Hi-C rocket flight. The full field-of-view of Hi-C is indicated by the yellow box. The green and black boxes $\beta$ and $\gamma$ indicate the plage area and the moss region displayed in panels $\beta$ and $\gamma$, respectively. Panel $(\boldsymbol{\beta})$ displays a zoom into the plage region south of the active region (46"x46"). The boxes 1 to 5 indicates features used for the thermal study in Sect.5.5.1. The box $\delta$ highlights the field-of view shown in Fig.5.3. Panel $(\gamma)$ shows the zoom into a moss region. As in panel $\beta$, the numbered boxes show the features for the thermal study.

a pixel of HMI. While magnetic field information at higher spatial resolution and magnetic sensitivity than that available with HMI would have been desirable, unfortunately, such data were not available for our region of interest, either from the ground or from the space-based Hinode observatory.

To study the possible presence of very hot plasma in relation to the small loop-like features, we used data from XRT that provides a spatial scale of $1^{\prime \prime}$ per pixel. During the Hi-C flight XRT, data were taken with the Ti-poly filter showing plasma in a broad temperature with a peak at approximately $9 \mathrm{MK}$. From the peak down to a temperature of $2 \mathrm{MK}$, the response of Ti-poly drops by a factor of approximately 15 (Golub et al. 2007, their Fig. 7). Unfortunately, the plage area of interest in our study is only partially covered by the XRT field-of-view. Still, the region connecting the plage to the main part of the active region is covered, which makes the XRT data very valuable for our study (see Sect. $5.2)$.

\subsection{Properties of small loop-like features}

Small loop-like features have been found in Hi-C observations by Peter et al. (2013). Based on a single image they found that these have a length of approximately $1.5 \mathrm{Mm}$ and a width below $200 \mathrm{~km}$, and they suggest an interpretation of them as miniature versions of coronal loops. The main purpose of our study is to investigate the spatial and temporal evolution and the thermal structure of these features and to relate them to the underlying magnetic field structure. We first discuss length, width and relative orientation with respect to the E-W direction of the Sun and horizontal motions of the loop-like structures as a function of time. 


\subsubsection{Identification of small loop-like features}

Identification of the loop-like features is via a combination of manual and automated procedures. Firstly, on each of the 25 images, we identified the 15"x15" subregion hosting the features of interest (Fig. 5.3). For each image, the intensity in that subregion of 15"x15" was normalised by the respective median value of intensity from that region. Then, the contrast of the subregion is enhanced by employing a median filter with a kernel size of $3^{\prime \prime}$.

We approximately identified four features by eye, marked A to D in Fig. 5.3. For each structure we defined a rectangular subfield only just covering this feature and its immediate surroundings (e.g. box 1 in Fig. 5.3 for feature A). In each of the small boxes, we calculated the maximum of the normalised and contrast-enhanced brightness, $I_{\max }$, during the time series. We then defined the small loop-like structures as the feature enclosed by the contour line at a level of $90 \%$ of $I_{\max }$ (cf. contours A to D in Fig. 5.3).

To describe the properties of the features we fitted an ellipse ${ }^{1}$ to the respective contour lines at a level of $90 \%$ of $I_{\max }$. This is motivated by the observation that the contour lines have an approximately ellipsoidal shape. The properties of the fitted ellipse in each time step was then used to characterise the loop-like features: the position of the ellipse represents the position of the loop feature; for its length and width we use the major and minor axis of the ellipse, the angle is defined as the angle of the major axis with solar-x, that is, the E-W direction and the brightness of each feature was calculated by the mean brightness within the ellipse. This was done independently for each of the exposures of the time series of full-frame Hi-C data so that we could also investigate the temporal evolution, at least over the section covered by more than two minutes of the full-frame data. To study the brightness variability we used images without contrast enhancement by the median filtering, of course.

The spatial extent of the loop-like features, as characterised by the ellipse contours at $90 \%$ intensity level, is a measure for the full width at half maximum of the features. While the plage region shows a peak intensity of a factor of approximately 10 higher than the surrounding quiet areas, the individual loop-like features have an intensity contrast of approximately $20 \%$ above the plage region in the immediate vicinity of them (see Fig. 4 of Peter et al. 2013). Therefore, the $90 \%$ level of the peak intensity (without background subtraction) represents the full width at half maximum. The values derived for the widths and lengths of the loop-like features can be lower limits only, because the background emission from the plage region might hide a larger (low intensity) extension of the features. However, this argument applies to any measurement of, for example, loop widths in the corona.

\subsubsection{Properties of small loop-like features}

The position of the features slowly changes in space, typically over slightly less than $1^{\prime \prime}$ over the $140 \mathrm{~s}$ covered by the full-frame images. The positions of the four features and their evolution in time is shown in Fig. 5.4. To check that there are no systematic residual image motions, we check the mean position (equal weighting) of the four intensity

${ }^{1}$ For ellipse fitting we use the function mpfitellipse from the IDL library of Craig Markwardt (https: //wWW . physics.wisc. edu/ craigm/idl/). 

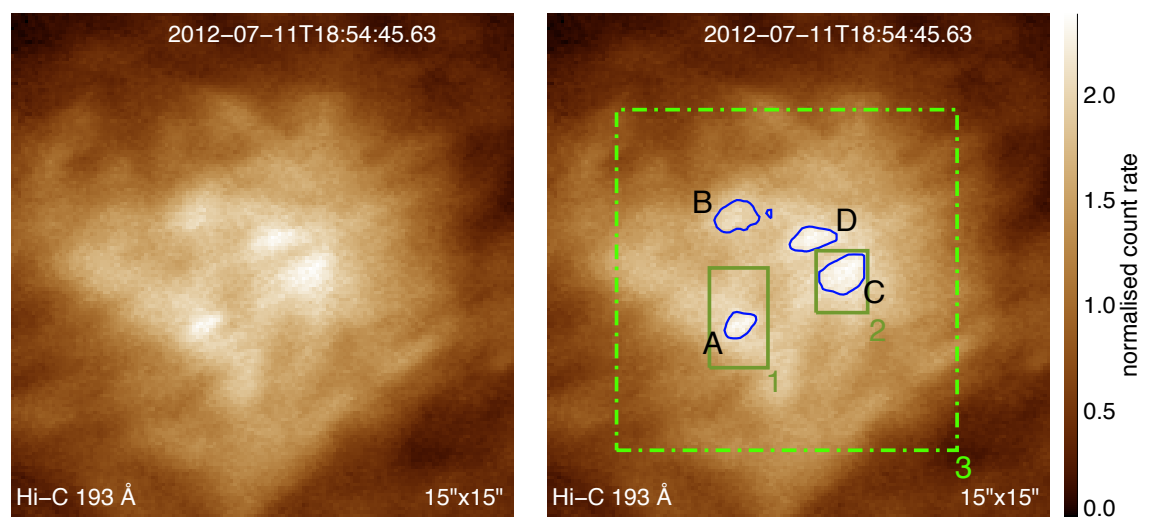

Figure 5.3: Zoom of the plage area seen by Hi-C (see box $\delta$ in Fig 5.2 $\beta$ ). We show the same image with and without annotation. The contours A to D show the miniature looplike features identified with the procedure outlined in Sect.5.3.1. The boxes 1 to 3 show the features for the thermal analysis in Sect.5.5.1 (same boxes as in Fig. 5.2 $\beta$ ).

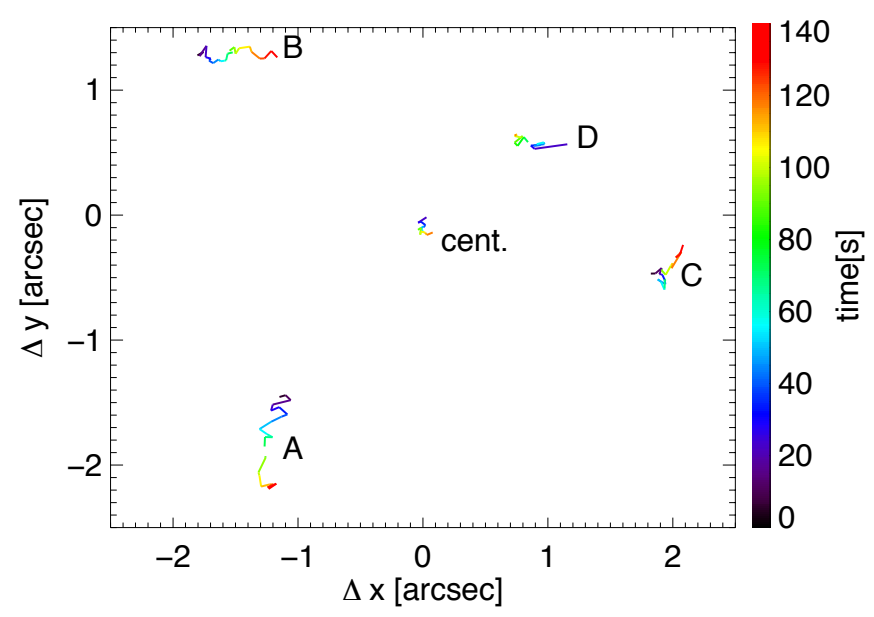

Figure 5.4: Horizontal motion of small loop-like features. The trajectories A to D show the centre of the respective features identified in Fig. 5.3 as a function of time (colourcoded) over 140s (after 18:54:11 UT). The error estimate for the position from the ellipse fitting procedure is approximately 0.5 pixels or 0.05 " (half a tick mark). For comparison, the trajectory labelled 'cent.' shows the mean position of the four loop-like features A to D. See Sect5.3.1. 

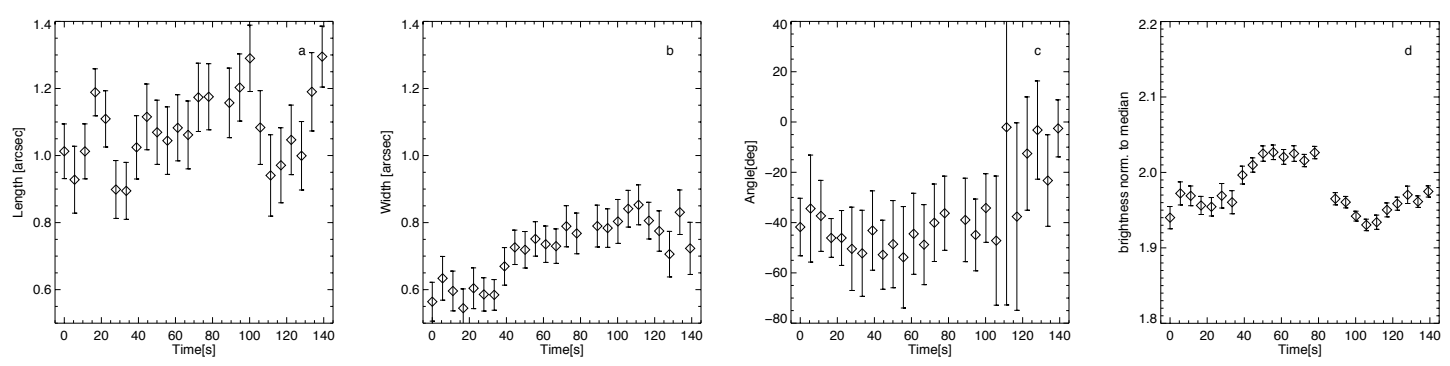

Figure 5.5: Temporal variation of the properties of one loop-like feature. This plot is for feature B as marked in Fig. 5.3. The properties of the other three features are shown in Figs. 5.10 to 5.12. These data are based on the ellipse fits of the features: (a) the major axis representing the length, (b) the minor axis the width, (c) the angle of the major semiaxis with the E-W direction for the angle, and (d) the intensity integrated over the ellipse for the structure brightness. The time is with respect to the first full-frame Hi-C image at 18:53:11 UT. See Sect5.3.1.

features that is found in the middle of Fig.5.4. This is almost stable with a motion of $<0.2^{\prime \prime}$ in $140 \mathrm{~s}\left(\cong 1.0 \mathrm{~km} \mathrm{~s}^{-1}\right)$. This reflects the good temporal alignment of the Hi-C data. The trajectories of the four features (A to D) cover approximately $1^{\prime \prime}$ during the observation in a more or less linear fashion. The motion of $\approx 1 " / 140$ s corresponds to a speed of $5 \mathrm{~km} \mathrm{~s}^{-1}$. That all four features move in random directions (as far as can be judged from four data points) underlines the idea that no systematic effects cause this result. In general, these structures exhibit random motions, which are consistent with the motion of the small-scale magnetic loops arching over granules discussed in the introduction. In particular, this horizontal velocity is consistent with observations of flux emergence on granular scales. As shown by Martínez González and Bellot Rubio (2009), in those cases, footpoints can be separated by approximately $2^{\prime \prime}$ within four minutes (see their Fig. 2), which would be approximately $6 \mathrm{~km} / \mathrm{s}$. Thus, the average velocity of the features we see is approximately comparable to average horizontal photospheric motions under small-scale flux-emergence conditions.

In general, while the structures move, they also (slightly) change their length and width. This is illustrated in Fig. $5.5 \mathrm{a}, \mathrm{b}$ for feature B (for the other features see appendix 5.8.1, Figs. 5.10 to 5.12). The typical length and width of the loop-like structures are $1 \mathrm{Mm}$ and $0.5 \mathrm{Mm}$, respectively, with a typical aspect ratio of approximately two. Strictly speaking, the footpoint distance of approximately $1 \mathrm{Mm}$ is only representative of the loop length if it is a low-lying (loop) structure (which we implicitly assume here). If it were a semi-circular loop, its length would be $\pi / 2$ times the footpoint distance, that is, some $50 \%$ longer.

Besides the horizontal motion of the features as a whole (Fig. 5.4), we also follow the motion of their endpoints (viz. footpoints) relative to their center. This is done by measuring the angle of the fitted ellipse with respect to the E-W direction. As an example, Fig. 5.5c shows the change of this angle during the Hi-C observation for feature B (again, other features in Figs. 5.10 to 5.12). Because of the considerable uncertainties in determining the angle (because the ratio of length to width is not big), the variation of the angle is almost within the error bars. Nevertheless, in three of the four cases we see a gradual 
rotation of the loop feature, with a difference between the maximum and minimum angles of approximately $\alpha \approx 40^{\circ}$ (expect the case in Fig. 5.10 c). While the question remains as to the significance of this value, we can at least estimate this as an upper limit. Considering the length of approximately $1 \mathrm{Mm}$, this angle corresponds to a rotational component of the motion of approximately $\sin \left(40^{\circ}\right) \times 1 \mathrm{Mm} \approx 0.6 \mathrm{Mm}$. Thus the upper limit of the speed of the footpoints during the 140s of the Hi-C full-frame data is approximately $4 \mathrm{~km} \mathrm{~s}^{-1}$. This is approximately consistent with the velocity of magnetic concentrations in the intergranular lanes derived from magnetic bright points (Jafarzadeh et al. 2014, their Fig. 2) and supports the interpretation that the footpoints are rooted in flux tubes in intergranular lanes.

Finally, we verified the variability of the brightness in time. For feature B this is shown in Fig. 5.5 d. There is a variation of approximately 5\% (for other features, even up to $15 \%$ ) of the brightness over approximately 100s. This variation of the brightness is significant in two respects. Firstly, this variability is approximately four times larger than the average error in brightness (taking into account the photon noise and the error in the ellipse fitting shown as bars in the plots). Secondly, this variation of 5\% to $15 \%$ is also significant when considering that the contrast of the loop-like features above the background of the plage is only some $20 \%$ (see end of Sect.5.3.1). This implies, that the change of the emission from the loop-like feature alone would change by $50 \%$ to almost $100 \%$ during the observation time. Still, the loop-like features are visible just above the background throughout the full Hi-C observation sequence of approximately two minutes. Naturally we can not draw a final conclusion on the lifetime of the loop-like features because the length of the time series of the full-frame Hi-C images is limited, but at least we can say that the lightcurve is consistent with a feature lifetime of two minutes or more. For the other features, the situation is less clear (Figs. 5.10 to 5.12). One feature shows almost no intensity variation, while the other two are caught while the intensity decreases during the observation time. We can thus conclude that the features probably have a lifetime of a few minutes or more.

Summarising these findings, the observed features show motions and lifetimes consistent with being anchored in magnetic concentrations in intergranular lanes. This would be consistent with the loop-like features being indeed miniature hot loops spanning one granule, and them being the result of rising magnetic flux tubes of the type reported by Ishikawa et al. (2010), for example. Furthermore their properties in terms of length, width and lifetime are comparable to the transition region loops at $0.1 \mathrm{MK}$ reported by Hansteen et al. (2014), therefore it might be that the features we see here are a coronal temperature version of those transition region loops.

However, based on the above information, it could still be that we see only one footpoint of a longer loop, similar to the scenarios discussed in the introduction (cf. Fig 5.1). For this, we discuss their relation to the magnetic field (Sect. 5.4) and the thermal structure (Sect.5.5).

\subsection{Relation to photospheric field}

For an investigation of how the loop-like features relate to the magnetic field, we carefully aligned the Hi-C images with the HMI magnetograms (for details of the alignment see Appendix 5.8.2). The accuracy of the alignment of the HMI magnetogram with the Hi-C 


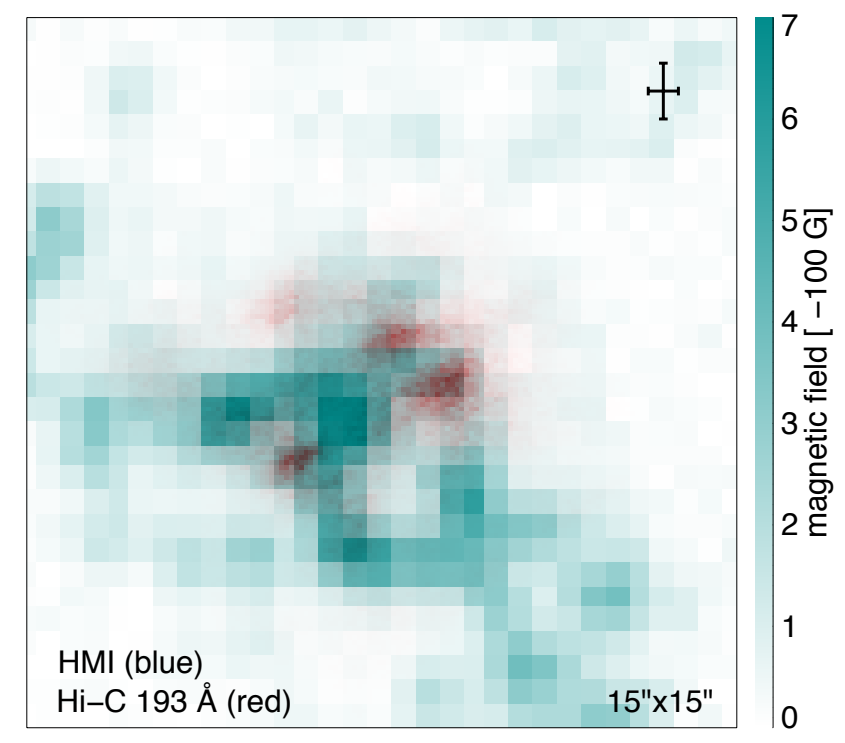

Figure 5.6: Aligned composite of photospheric magnetogram and coronal emission. In the same field-of-view as in Fig. 5.3 we plot the magnetogram on a blue scale and overlay the coronal image from $\mathrm{Hi}-\mathrm{C}$ in red. The magnetogram in this field-of-view contains only one single magnetic polarity. The Hi-C image is the same as in Fig.5.3, but on a non-linear colour scale to emphasise the loop-like features. See Sect.5.4.

image is within less than half a pixel of HMI, at approximately $0.2^{\prime \prime}$. The result of the alignment is displayed as a composite in Fig. 5.6 showing the magnetogram along with the Hi-C image of coronal plasma over-plotted in red.

Within the sensitivity and resolution of HMI, only one polarity is seen in the magnetogram of this plage region, as noted already by Peter et al. (2013) in their study of this same region. In the field-of-view in Fig. 5.6 not a single pixel of the HMI magnetogram shows an opposite polarity. However, this does not exclude the presence of small parasitic opposite polarities. At higher spatial resolution and magnetic sensitivity, one might well see opposite polarities not visible to HMI. For example, data from the Imaging Magnetograph eXperiment (IMaX; Martínez Pillet et al. 2011) on the Sunrise balloon telescope (Solanki et al. 2010), at much higher resolution and sensitivity than HMI, clearly show small-scale magnetic patches of opposite polarity close to a largely unipolar region (e.g. Wiegelmann et al. 2013, their Fig. 2) that would not be detectable by HMI. Unfortunately, as discussed in Sect.5.2, no observations of higher resolution or sensitivity are available for this region of interest.

The comparison of the Hi-C image and the magnetogram in Fig. 5.6 shows that typically at least part of the loop-like feature is located above a concentration of magnetic field. According to the scenarios in Fig. 5.1 this would support the theory that one (or the only) footpoint is rooted in the photospheric magnetic field concentration. Because of the arguments concerning weak, small and opposite-polarity patches, the missing detection of HMI of a possible second footpoint cannot be used as an argument against the small-scale loop scenario. Therefore this discussion of the relation of the magnetic field to the emission seen in $\mathrm{Hi}-\mathrm{C}$ must remain indecisive and we must rely on the analysis of 


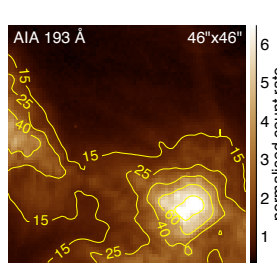

$\log T=6.1$

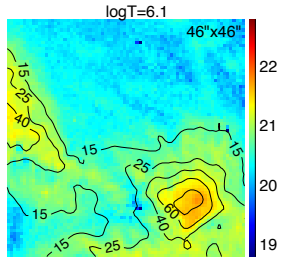

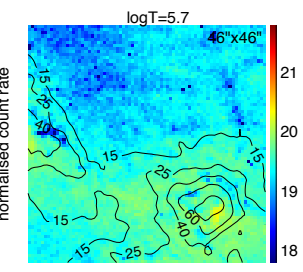

$\log T=6.2$

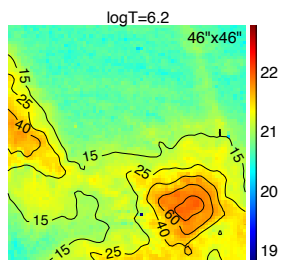

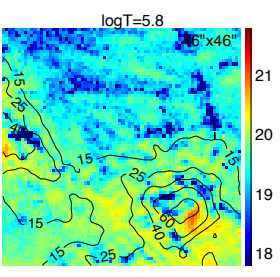

$\log T=6.3$

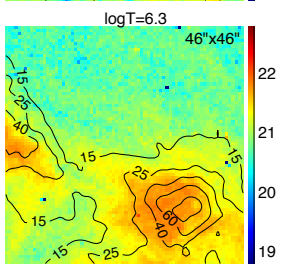

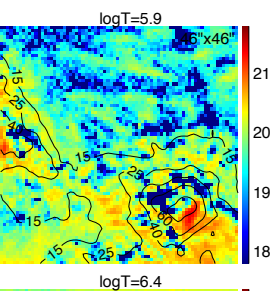
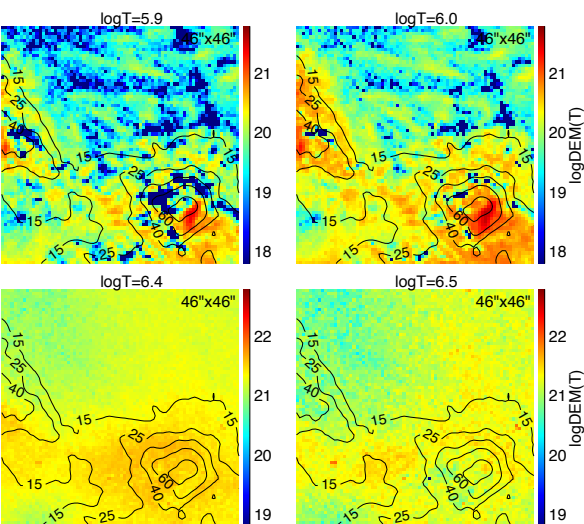

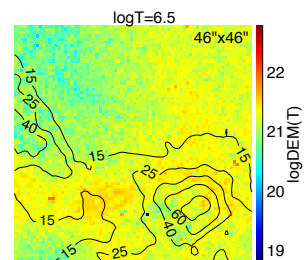

Figure 5.7: Coronal image and thermal structure. The top left panel shows the AIA image at $193 \AA$. The other panels display spatial maps of the differential emission measure (DEM) at different temperatures $T$ labeled by $\log T[\mathrm{~K}]$. All panels show the same field-of view that is identical to Fig. 5.2 3 . The plage region with the miniature loop-like structures is in the bottom-right part. For a better comparison between the panels we add contour lines of the brightness in the AIA $193 \AA$ channel to all the panels. The numbers with the levels denote the percentage of the peak $193 \AA$ brightness in this field-of-view. All DEM maps have the same dynamic range of $10^{4}$ although their lower (and upper) limits change to best represent the DEM structures. See Sect.5.5.1.

the thermal structure at and around the plage region hosting the small loop-like features.

\subsection{Relation to thermal structure}

There is a significant difference between a miniature coronal loop and the moss emission that might also appear as an elongated structure (Fig.5.19,b): by its very nature in the vicinity of the moss structure there should be some signature of the hot loop that is connected to the moss region, while for an isolated miniature loop one would not expect that hot component of the emission. To address this difference, we investigate the differential emission measure (DEM; Sect.5.5.1) and the X-ray emission (Sect.5.5.2) from the plage region hosting the small loop-like features and compare it to a moss area.

\subsubsection{Differential Emission Measure (DEM)}

We perform a DEM analysis in the vicinity of the loop-like features and compare this to a moss region that has been analysed before (Testa et al.2013) as well as to quiet regions without notable emission in the $193 \AA$ band for a blind test, all in the Hi-C field-of-view. The DEM provides some information on how the plasma is distributed in temperature throughout the atmosphere (along the line-of-sight) and is defined as

$$
D E M=n_{e}^{2}\left(\frac{d T}{d h}\right)^{-1} \text {. }
$$

Here $n_{e}$ is the electron density, $T$ the temperature, and $h$ the height along the line-ofsight. Being a function of $T$, the DEM is a measure for how much plasma is present 
at temperatures where the EUV (and X-ray) emission is originating. Hi-C alone can not be used to calculate the DEM because it provides only one wavelength band. In contrast, AIA includes a sufficient number of bands spanning the typical temperatures in the corona, albeit at a significantly lower spatial resolution. So we use AIA imaging data (see Table 5.1) to perform an inversion resulting in maps of the DEM over the covered temperature range. This analysis is based on a set of near-simultaneous images obtained between 18:53:56 and 18:54:06, one in each channel. In our analysis we took into account photon noise and readout noise. $2^{2}$

There are numerous publicly available methods to perform this inversion, and we decided to use the regularised DEM inversion by Hannah and Kontar (2012) for its robustness.

To obtain DEM curves for each region-of-interest (see boxes 1-9 in Fig. 5.2 $\beta, \gamma$ ) we calculated the average intensity in each region-of-interest for each AIA channel. The inversion procedure as described by Hannah and Kontar (2012) uses these AIA count rates as input and provides the DEM as a function of temperature, including error estimates for the temperature and the DEM (shown as a cross in Fig.5.8). One contribution to the errors in the DEM are the uncertainties in the count rates of the AIA channels (with counts typically ranging from $50 \mathrm{DN} / \mathrm{pixel} / \mathrm{s}$ in the weak channels (e.g. $94 \AA$ ) to a few thousand DN/pixel/s in the stronger channels (e.g. $193 \AA$ ). However, the errors returned by the procedure also include uncertainties of the method and the width of the contribution functions of the AIA channels in temperature (for details see Hannah and Kontar 2012).

The maps of the DEM in the vicinity of the small loop-like structures (Fig. 5.7) show a clear signature of an enhanced DEM at temperatures around $1.5 \mathrm{MK}(\log T=6.2$; here and in the following, all $\log T$ values refer to $T$ in units of $\mathrm{K}$ ). This simply reflects the enhanced emission we see in the Hi-C passband. In particular, the DEM maps do not show any significant signal at higher temperatures.

To emphasise this result from the spatial maps, we check the DEM curves as a function of $T$ for selected regions (Fig. 5.8). In the areas directly above two of the loops (regions 1 and 2 in Figs. $5.2 \beta$ and 5.3) as well as in a larger area encompassing all the small looplike features in the plage region (region 3 in Figs. $5.2 \beta$ and 5.3 we see a clear peak at approximately $\log T=6.2$. The drop to lower and higher temperatures is comparably sharp. In particular, the drop to higher temperatures distinguishes the loop-like features from the moss area (see below). As expected, the DEM from the plage region is significantly higher than in sample regions covering the quiet regions (see Fig. 5.8). In particular, also region 5 in Fig. 5.2 $\beta$, that is located at a faint longer loop seemingly connecting the plage area with the main part of the active region to the North, does not show any enhanced DEM at higher temperatures, at least not higher than the DEM curves for the plage regions 1 to 3 . This is why we labelled this region 5 as 'quiet', as well, in Fig.5.8.

To confirm that this technique would detect the presence of hot plasma in moss regions, we checked the moss region in the Hi-C field-of-view that has been investigated already by Testa et al. (2013). These are regions 6 to 9 in Fig. 5.2 $y$. The DEM curves of these regions in Fig.5.8 are distinctively different from the loop-like features in the plage. While the DEM of the moss regions is comparable around $\log T=6.2$, where the plage region peaks, only the moss regions show a clear enhancement of the DEM up to at least

\footnotetext{
${ }^{2}$ To calculate the photon noise and the read noise of the SDO/AIA images we use the procedure aia_bp_estimate_error available in SolarSoft (http://wWw.lmsal.com/solarsoft/).
} 


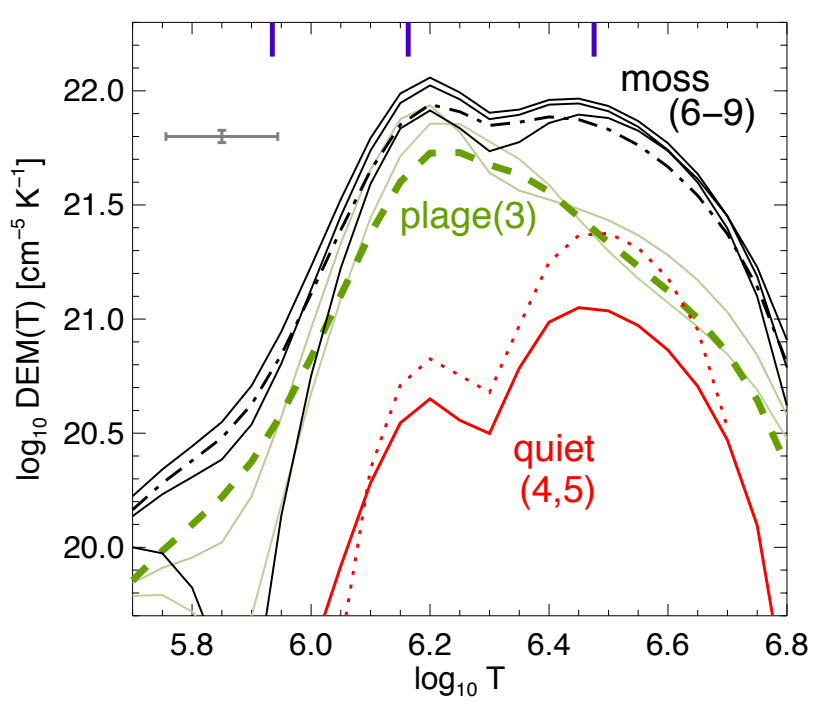

Figure 5.8: Differential emission measure (DEM) distribution for different structures. The labelling of the lines corresponds to the regions marked in Fig. 5.2 $\beta, \gamma$. The green lines represent two individual small loop-like features (thin green; 1,2) and an average covering the whole plage area hosting the small loop features (thick dashed green; 3 ). For comparison the red lines are the inversions for the quiet regions $(4,5)$. The black lines show the DEM in the moss areas (6-8), with the thick dashed line for the average over the larger moss region (9). The error bars (grey) present the average uncertainties for all curves in $\log \mathrm{T}$ range from 6.0 to 6.7. The three blue markers at the top axis indicate the temperature of the peaks of the DEM components in coronal holes, quiet Sun, and active region according to Landi and Feldman (2008). See Sect.5.5.1.

$\log T=6.6$.

Therefore we conclude that there is no hot plasma present in the vicinity of the looplike features. At least not to the same extent as in moss regions. To further verify this, we also investigated the X-ray emission.

\subsubsection{X-Ray observations}

Including X-ray observations can help to relax the limitation of AIA in terms of temperature coverage. While AIA alone can provide the DEM only up to approximately $\log T=6.6$ or 6.7 ( $\approx 5 \mathrm{MK}$; cf. Fig. 5.8), XRT observations cover a broad range of temperatures centered at almost $10 \mathrm{MK}$ (cf. Table 5.1). In principle one could include X-ray observations in the DEM inversion (e.g. Cheung et al. (2015)). Here we choose the more straight forward way and study the X-ray images directly. This provides information if a hot component of a loop rooted in the loop-like features is present that might not be revealed by AIA. The DEM, as derived from AIA in the plage region hosting the small loop-like features, drops steeply above $\log T=6.3(\approx 2 \mathrm{MK})$. The response of the Ti-Poly filter of XRT drops by a factor of (only) approximately 15 from its peak (at $9 \mathrm{MK}$ ) to $2 \mathrm{MK}$ (Golub et al. 2007). Therefore, XRT is well suited to test if there is a hot component of plasma that would go unnoticed by the DEM analysis of AIA. The hot emission from X-rays in the 

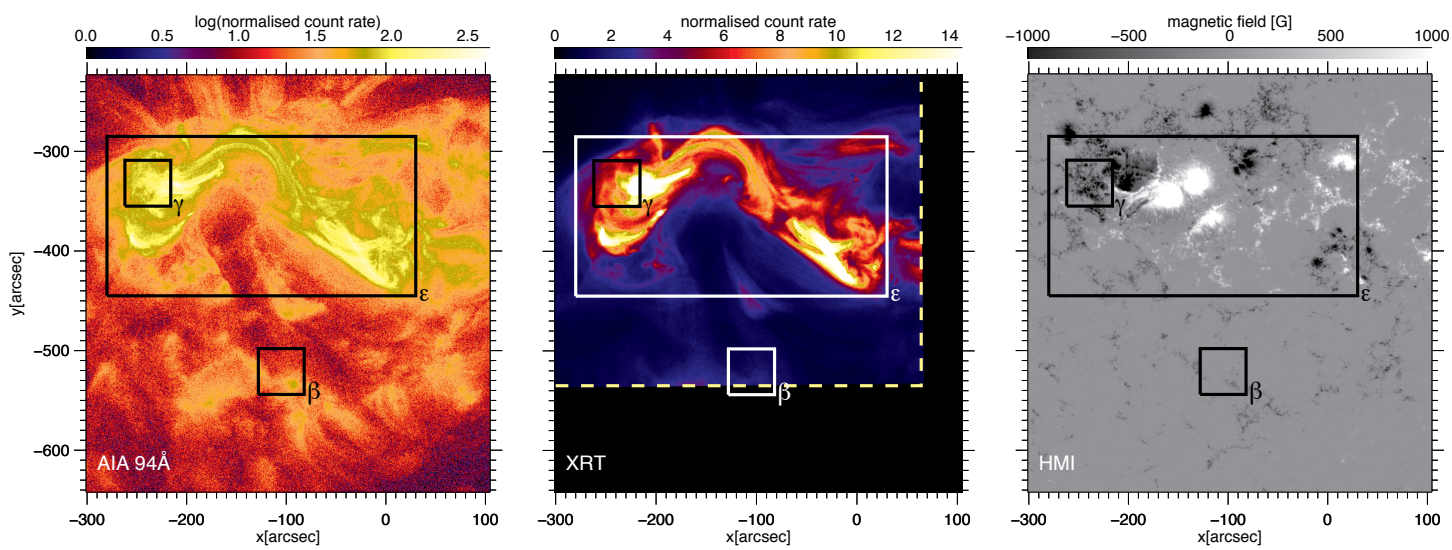

Figure 5.9: Emission from hot plasma and underlying magnetic field. The left two panels show the emission in the AIA channel at $94 \AA$ and the Ti-poly channel of XRT on Hinode. The XRT image is taken during the Hi-C flight (at 18:54:48 UT). The right panel shows the HMI magnetogram. All panels cover the full field-of-view of the Hi-C data (cf. large rectangle in Fig. $5.2 \alpha$ ). The box $\beta$ indicates the region with the plage area hosting the small loop-like features as also displayed in Fig. 5.2 $\beta$. The rectangle $\varepsilon$ outlines the area covering hot coronal loops and moss as investigated by Testa et al. (2013). In their paper they display this region $\varepsilon$ rotated counter-clockwise by $90^{\circ}$ The moss area displayed in Fig. 5.2 $y$ and marked here also by $\gamma$ roughly corresponds to the moss region M3 of Testa et al. (2013), their Fig. 2. The yellow dashed line in the XRT image marks the edge of the XRT field-of-view. See Sect.5.5.2

Hi-C field-of-view as seen by XRT on Hinode is displayed in Fig.5.9. For comparison we show the AIA $94 \AA$ channel that reveals comparably hot plasma (cf. Table 5.1), hotter at least than $2 \mathrm{MK}$ and colder than $12.5 \mathrm{MK}$. In this context we used the AIA $94 \AA$ channel (together with AIA $193 \AA$ ) simply for aligning XRT with Hi-C.

One problem concerning the XRT data during the Hi-C flight is that they do not fully cover the whole field-of-view of Hi-C. In particular, they only partially cover the plage region hosting the loop-like features. Still, the XRT image shown in Fig.5.9, which is taken during the Hi-C flight, fully covers the region between the negative magnetic polarity of the plage region and the positive polarity in the main part of the active region to the North. This is clear from the comparison to the HMI magnetogram in Fig. 5.9. Therefore XRT covers the region where one would expect the hot loops that might be rooted in the plage region ( $\beta$ in Fig. 5.9).

In the space covering the connection from the plage area with the small loop-like features to the main part of the active region, there is no significant $X$-ray emission visible (Fig. 5.9). While we see clear hot loops in X-rays in the main part of the active region that contains the moss regions already studied by Testa et al. (2013), such X-ray emission is not related to the plage region ( $\beta$ in Fig. 5.9). In fact, the region North of the plage region, where the magnetic connection would be expected, is particularly dark in X-rays. This extends the temperature range of the missing hot plasma related to the plage region with the small loop features and highlights the lack of significant amounts of plasma present at higher temperatures from above $2 \mathrm{MK}$ up to $10 \mathrm{MK}$.

With these considerations on the thermal structure based on the DEM and the X-ray 
emission, we conclude that it is unlikely that the loop-like features are the footpoints of hot (and dense) loops. Therefore, we exclude the moss scenario shown in Fig. 5.1p and discussed in the introduction.

\subsection{Discussion}

The above discussion of the thermal structure suggests that the loop-like features are not the moss-like footpoints of hot dense loops. This argument is based on the absence of a significant amount of hot plasma that would fill the related loop. Still, one could speculate that the scenario in Fig. 5.1k could apply; namely that the small loop-like features are at the footprints of larger (hot) but dilute loops (cf. Sect.5.1). Heat input from below could heat the lowermost part, but leave the upper part of the loop unaffected. In this case, the longer loops would not be visible in X-rays or leave a signature in the DEM at high temperatures, while we still see the compact loop-like features appearing at their footpoints. In principle, this would be consistent with the thermal structure we discussed in Sect.5.5,

However, there are theoretical arguments against this interpretation of the small looplike features being footpoints of long dilute loops. To illustrate this we conduct a Gedanken experiment: We assume that along a magnetic fieldline with a length of several tens of $\mathrm{Mm}$ close to the footpoint exists a dense structure at approximately $1.5 \mathrm{MK}$ with a length of approximately $1 \mathrm{Mm}$ along the fieldline. Above that, the loop is hotter but very dilute ${ }^{3}$ This structure would be, in general, consistent with our observation (according to the scenario in Fig. 5.1 c). We would see an elongated feature with a length of $1 \mathrm{Mm}$ and with a peak of the DEM at approximately $\log T \approx 6.2$ or $1.5 \mathrm{MK}$ (cf. Fig. 5.8), but no Xray emission above, because the hot part of the loop is not dense enough. However, the thermal stratification assumed for this setup would not be stable over the time of one to two minutes when we observe the features during the Hi-C flight. The dense plasma at a temperature of $\log T \approx 6.2$ or $1.5 \mathrm{MK}$ has a pressure scale height of approximately $75 \mathrm{Mm}$ (or 0.1 solar radii). With no (significant amount of) dense plasma above it, the dense 1.5 MK plasma would quickly expand into the upper part of the loop, similarly to an expansion into vacuum. Expansion into vacuum happens at approximately the adiabatic sound speed, which for plasma at $1.5 \mathrm{MK}$ is approximately $185 \mathrm{~km} \mathrm{~s}^{-1}$. Even when assuming an expansion speed much less than that, $100 \mathrm{~km} \mathrm{~s}^{-1}$, for example, within the timescale during which these structures exist (and we observe them), that is, approximately $100 \mathrm{~s}$, they should have expanded by approximately $10 \mathrm{Mm}\left(=100 \mathrm{~km} \mathrm{~s}^{-1} \times 100 \mathrm{~s}\right)$. This would be a distance much larger than the size of the structures, an expansion we should clearly see (e.g. in the form of a jet). This contradicts our initial assumption of 1.5 MK dense plasma below a hot dilute loop, because we do not see any significant expansion of the small loop-like features. This leads us to the conclusion that they cannot be the footpoint regions of large dilute loops.

\footnotetext{
${ }^{3}$ Alternatively, the upper part of the loop could also be cool, say at only $10^{4} \mathrm{~K}$. Because the Barometric scale height at low temperatures (at $10^{4} \mathrm{~K}$ it would be only $500 \mathrm{~km}$ ) is small compared to the loop length, also in this case the loop would be at very low density in its upper part. Therefore, for a cool upper part of the loop the arguments would be the same as for a dilute hot upper part discussed here, and our conclusion would be identical.
} 
This leaves only one scenario, namely that the small loop-like features are indeed tiny versions of hot coronal loops, as depicted in Fig. 5.1 a. The main argument against this could be that in the HMI magnetograms we see only one single polarity in the plage region hosting these structures, as already pointed out by Peter et al. (2013). However, as discussed in Sect.5.4, these HMI observations of only one single polarity do not rule out the possibility of small-scale opposite polarities, which is because of the limited sensitivity and spatial resolution; instruments with higher resolution and sensitivity have seen such small-scale opposite polarities (cf. Sect.5.1), even though no such observations are available during the Hi-C flight for the area we investigate.

There is also observational evidence for small loops at coronal temperatures through data from AIA; albeit those loops are significantly longer than the miniature loops we report here. Wang (2016) finds short coronal loops in AIA $171 \AA$ observations in plage regions that have footpoint distances from $3 \mathrm{Mm}$ to $5 \mathrm{Mm}$. This implies that their lengths are of the order of $5 \mathrm{Mm}$ to $10 \mathrm{Mm}$, depending which loop geometry is assumed. These loops also occur in plage regions that appear unipolar in HMI, and Wang (2016) uses this as indirect evidence for small-scale opposite polarity magnetic field hidden to HMI. In some way, the small loops in plages found recently by Wang (2016) could be considered as longer versions of the structures we study here in some detail and were first reported by Peter et al. (2013). In terms of modelling, it would be interesting to see what onedimensional loop models would predict for very short loops that are heated to $1.5 \mathrm{MK}$ or more, if that is possible. The models by Sasso et al. (2012) produce transition region loops with temperatures well below $1 \mathrm{MK}$ only. If, in those models, the energy input is increased, the loops might reach higher temperatures, and it would be interesting to see if they turn out to be stable or highly intermittent.

\subsection{Conclusions}

We investigated small loop-like features in the solar corona seen in the $193 \AA$ channel of Hi-C. They have typical lengths of $1 \mathrm{Mm}$ and lifetimes of some 100s. They appear in a plage or enhanced network region at the periphery of an active region. The analysis of the morphology of these structures in terms of horizontal motions and lifetime suggests a close relationship with the granular motions in the photosphere (Sect.5.3.2). One end of the loop-like features is rooted in a magnetic field concentration as seen by HMI. The limited resolution and sensitivity of HMI prevents definite conclusions if the other end of the feature is rooted in an opposite magnetic polarity (Sect.5.4).

The analysis of the thermal structure of the loop-like features (Sect.5.5) shows that they cannot be the footpoints of hot dense loops, as is the case for moss (cf. Fig.5.1p). This is supported by a DEM analysis (Sect. 5.5.1) as well as by the direct X-ray observations and a comparison to actual moss structures (Sect.5.5.2). Theoretical arguments can be made that the loop-like features are also not at the footpoints of (hot) dilute loops (cf. Fig 5.1c) heated from their footpoints, because then we would expect to observe expanding (jet-like) features, which we do not see (Sect.5.6.

This discussion leaves us interpreting the loop-like features as actual miniature coronal loops (cf. Fig 5.1 a). These would span just a single granule and connect a magnetic concentration in an intergranular lane with a feature of opposite-polarity on the other side 

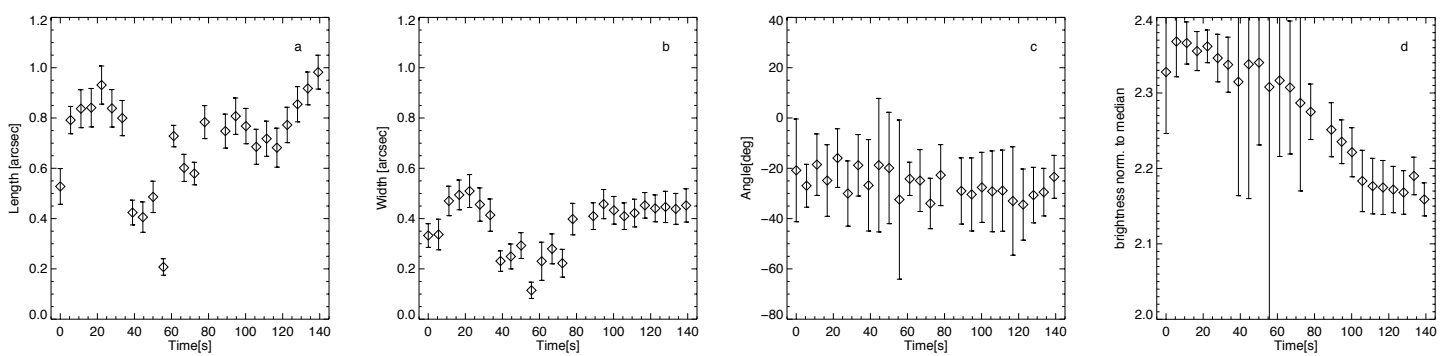

Figure 5.10: Properties of loop-like feature A marked in Fig.5.3. Same format as Fig.5.5

of the granule. That HMI does not see a parasitic opposite polarity does not exclude this scenario, because high-resolution photospheric magnetic field observations (e.g. Martínez González and Bellot Rubio 2009, Wiegelmann et al. 2013), as well as indirect evidence from coronal structures (Wang 2016), provide evidence for the existence of such smallscale opposite polarities (Sects.5.4 and 5.6).

These miniature loops might well be related to small-scale flux emergence events, where small magnetic fluxtubes break through the photosphere (e.g. Ishikawa et al. 2010). Clear evidence for such short $(1 \mathrm{Mm})$ loops reaching transition region temperatures (i.e. approximately $0.1 \mathrm{MK}$ ) has been reported recently using IRIS data (Hansteen et al.2014). It does not seem unrealistic that, in cases of stronger heating, such loops might reach higher coronal temperatures of more than $1 \mathrm{MK}$. The transition region loops are visible by IRIS because of its high spatial resolution of approximately $0.3^{\prime \prime}$. To see such miniature loops in the corona, one needs comparable spatial resolution, which is not available with the current workhorse of coronal imaging, AIA, but is made available by the rocket experiment Hi-C that we used here. For this (and for other reasons) we hope for a reflight of Hi-C.

\subsection{Appendix}

\subsubsection{Properties of individual loop-like features}

In Sect.5.3.2 we discussed the properties of the loop-like structures in terms of length, width, orientation and brightness. There we mainly concentrated on one single structure, namely feature B marked in Fig. 5.3. Here we add the corresponding properties for the three additional structures, A, C and D, as marked in Fig.5.3. In the same format as used in Fig. 5.5 for the time evolution of the properties of feature B, we show here in Figs. 5.10 to 5.12 the properties of features $\mathrm{A}, \mathrm{C}$ and $\mathrm{D}$.

The properties of all four features are approximately similar, except for the evolution of the brightness. We could follow only feature B over a full life-cycle, that is, from an increase of the brightness until it faded away again within less than 2 min (cf. Fig. 5.5d). The three other features shown here are seen either only in the declining phase (Figs. 5.10 and 5.11) or show an almost constant brightness (Fig. 5.12). 

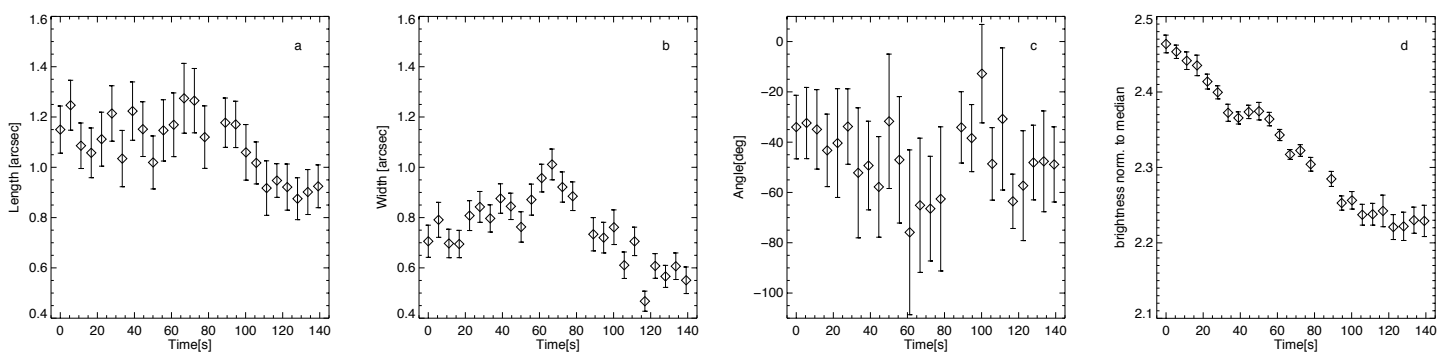

Figure 5.11: Properties of loop-like feature C marked in Fig.5.3. Same format as Fig.5.5
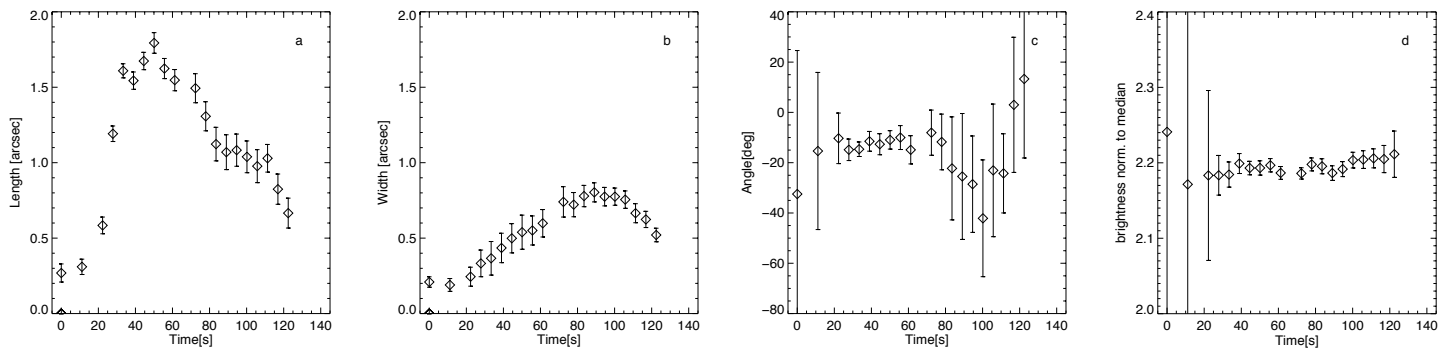

Figure 5.12: Properties of loop-like feature D marked in Fig.5.3. Same format as Fig.5.5

\subsubsection{Spatial alignment of $\mathrm{Hi}-\mathrm{C}$ with magnetogram}

To investigate the relation of the small loop-like coronal structures to the magnetic field in the photosphere we have to perform an alignment through different steps. The result of this alignment procedure is shown in Fig. 5.6 and discussed in Sect.5.4. In this appendix we briefly describe the alignment procedure.

For the alignment chain we use a subregion of approximately $100^{\prime \prime} \times 100^{\prime \prime}$ with the plage region hosting the small loop-like structures at approximately the center. All images used for the alignment are scaled (through interpolation) to the pixel scale of Hi-C. We first align the Hi-C image to the AIA image in the same $193 \AA$ channel. Because these images show the same coronal emission, only at different spatial resolution, this alignment is very reliable. The next step is to align the AIA $193 \AA$ image to the AIA $131 \AA$ channel. While the temperature of the peak contribution is quite different (cf. Table 5.1), both channels also have a significant contribution from lower (transition region) temperatures from $\log T=5.4$ to 5.6 (cf. Boerner et al. 2012). Therefore, the plage and enhanced network regions around our region-of-interest show many similar structures in the emission in the $193 \AA$ and the $131 \AA$ that provide a good basis for the alignment. This is also the case for the next step when aligning the AIA $131 \AA$ channel with the AIA $1600 \AA$ images that show the low chromosphere, because in both channels the emission from the (enhanced) network will dominate. In the final step we align the AIA $1600 \AA$ image with the HMI magnetogram. This alignment procedure is illustrated in Fig.5.13, where we show the respective images together with contour plots of the image in the next step of the alignment procedure.

For all alignment steps we use a cross-correlation technique to determine the misalignment and then shift the images with sub-pixel accuracy. We test the accuracy of the 
alignment through an alignment of the final aligned images. In Table 5.2 we list the misalignment (separate for the solar $x$ and $y$ directions) of all alignment steps. Combined, these give a misalignment of $0.2^{\prime \prime}$ and $0.3^{\prime \prime}$ in the $x$ and $y$ directions, respectively. This alignment accuracy is of the order of the spatial resolution of $\mathrm{Hi}-\mathrm{C}$, or a fraction of a pixel of HMI or AIA, just as one would expect. This emphasises the fact that we can trust the spatial alignment between Hi-C and the HMI magnetograms, which is the basis for the discussion on the relation of the small loop-like features to the magnetic field in Sect.5.4.

Table 5.2: Accuracy of spatial alignment in solar $x$ and $y$ directions.

\begin{tabular}{ccc}
\hline \hline images & $x\left[{ }^{\prime \prime}\right]$ & $y\left[{ }^{\prime \prime}\right]$ \\
\hline HMI $\leftrightarrow$ AIA 1600 & 0.06 & 0.13 \\
AIA 1600 $\leftrightarrow$ AIA 131 & 0.10 & 0.24 \\
AIA 131 $\leftrightarrow$ AIA 193 & 0.13 & 0.18 \\
AIA 193 $\leftrightarrow$ Hi-C & 0.01 & 0.01 \\
\hline
\end{tabular}



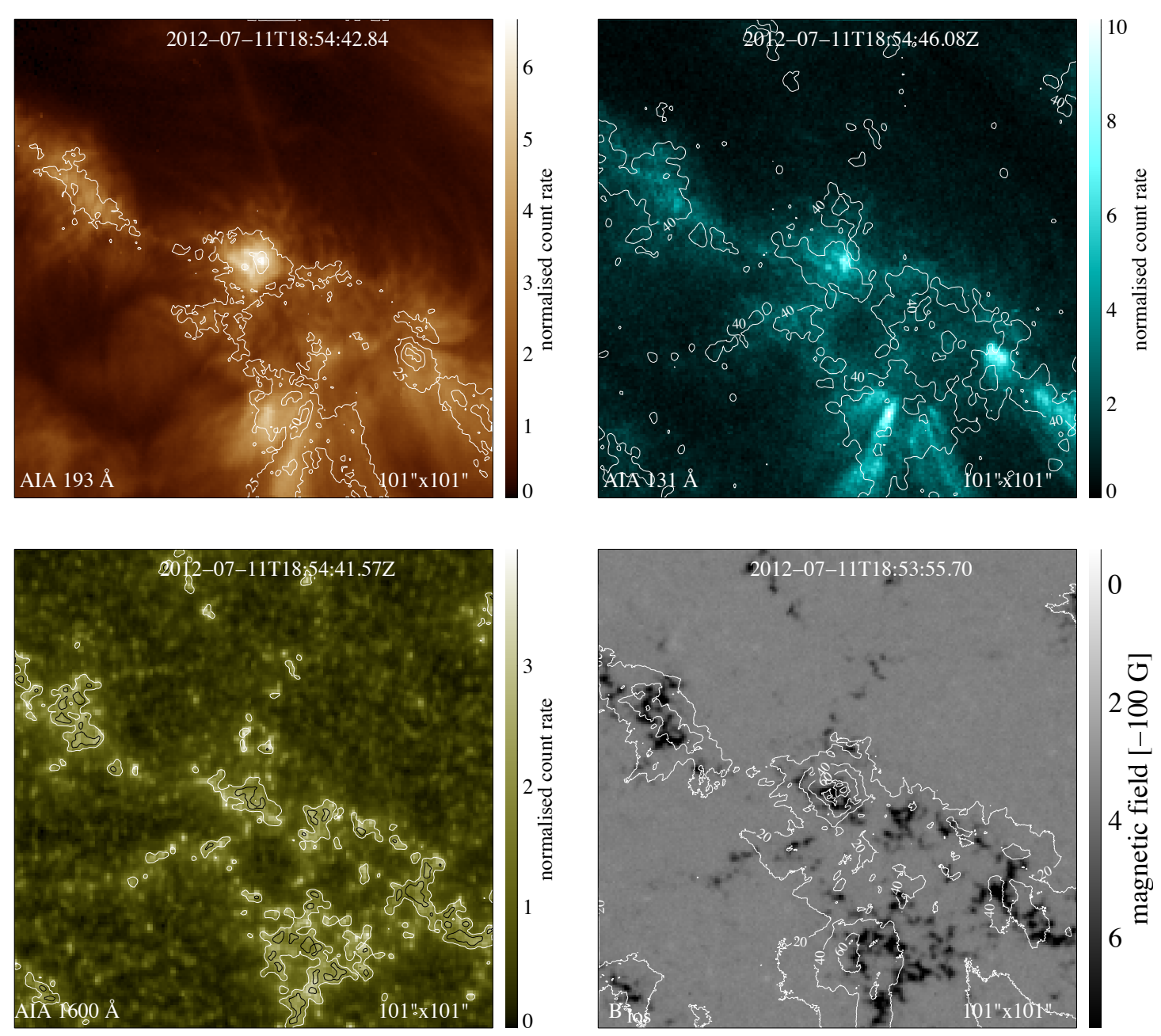

Figure 5.13: Illustration of the alignment procedure. The panels show the sequence of $101^{\prime \prime} \times 101^{\prime \prime}$ images of the alignment chain, that is, AIA $193 \AA \rightarrow$ AIA $131 \AA \rightarrow$ AIA $1600 \AA \rightarrow$ HMI magnetogram. In each image the contours of the respective image following in the chain are overplotted. The Hi-C $193 \AA$ image is aligned with the AIA $193 \AA$ channel and its contours are overplotted on the HMI magnetogram. The magnetogram contours are at $-100 \mathrm{G}$ (white) and $-300 \mathrm{G}$ (black). The other contours show the percentages of the maximum brightness in the respective channel (numbers with the contour lines). See Appendix 5.8.2 



\section{The small-scale structure in the transition region}

* This chapter is based on a journal article that is in preparation.

\subsection{Introduction}

The transition region and the solar corona are rich in many types of structures which cover a large range of sizes, lifetime and fast dynamics. The transition region, an interface between the significantly cooler chromosphere $\left(10^{4} \mathrm{~K}\right)$ and the hot corona $(1 \mathrm{MK})$, plays an important role in the mass and energy transport in the solar atmosphere. The smallscale structures which are numerous in the upper solar atmosphere, can have a significant influence on these processes. Therefore, the investigation of those structures is critical to better understand the upper solar atmosphere.

Loops are the most characteristic structures of the transition region and the solar corona. The small-scale $(<4 \mathrm{Mm})$, cool $\left(<2 \times 10^{5} \mathrm{~K}\right)$ loops were first observed by Porter et al. (1987). For temperatures from $2 \times 10^{5} \mathrm{~K}$ to $1 \times 10^{6} \mathrm{~K}$, Dowdy (1993) proposed intranetwork loops, with size about $\sim 10 \mathrm{Mm}$, rooted in areas of two opposite magnetic polarities. However, in the transition region exist also several different types of small-scale structures. Warren and Winebarger (2000) presented the small-scale loop-like structures that do not connect opposite magnetic polarity. The open question is whether these smallstructures are really unipolar or if they are supported by unresolved opposite magnetic polarity (Chitta et al. 2016). High-spatial and temporal resolution instruments, such as Hinode (Tsuneta et al. 2008), the High Resolution Coronal Imager (Hi-C; Cirtain et al. 2013) and the Interface Region Imaging Spectrograph spacecraft (IRIS; De Pontieu et al. 2014) open a new era of the study of small-scale structures. Winebarger et al. (2013) showed the cool $\left(\sim 10^{5} \mathrm{~K}\right)$, short living $(\sim 70 \mathrm{~s})$, small-scale loops $(4.8-7.5 \mathrm{Mm})$ build of $\left(\sim 10^{10} \mathrm{~cm}^{-3}\right)$ dense plasma. Based on IRIS data, Hansteen et al. (2014) studied the very rapid (few minutes lifetime), small-loops with a length in the range 4-12 Mm. Using Hi-C observations, Peter et al. (2013) found miniature loops. Barczynski et al. (2017) showed that these structures with a length of $1 \mathrm{Mm}$ and lifetime less than 2 minutes reach coronal temperatures. In one of their scenarios, these loops emerge to the corona from a lower part of the solar atmosphere, therefore they should be also visible in the transition region temperatures.

Naturally, several questions arise; how do the small-scale structure evolve? what is the role of the magnetic field in that process? What determines the size and lifetime of the small-scale structures? The simultaneous observation of the intensities and magnetic 
field obtained by high-resolution instruments are a good starting-point to begin the search of the answer to the above questions.

In this chapter, we concentrate on the temporal evolution of the small-scale structures in the quiet Sun. We focus on selected events, such as the small-scale loops and UV intensity response of two similar magnetic field patches. To this end, we use simultaneous observations obtained by IRIS and Hinode. These instruments provide the high-temporal $(\sim 30 \mathrm{~s})$ and spatial $\left(0.16^{\prime \prime}\right.$ pixel $\left.^{-1}\right)$ resolution that allows to study the dynamics of smallscale structures in terms of intensity and the underlying magnetic field variability. More precisely, we studied the geometrical properties, dynamics, density, and the relation between emission and the magnetic field.

Our aim is to understand how the magnetic fields have an influence on the temporal evolution of the small-scale structures as well as what is the relation between small-scale structures observed in the transition region and the underlying magnetic field. In this work, we present observations and methods of data preparation (Sect.6.2), the description of structures (Sect.6.3) and discuss the scenarios which explain of how these structures evolve (Sect.6.4).

\subsection{Observation and data preparation}

\subsubsection{Observations}

In this study, we concentrate on the simultaneous observation obtained by the Interface Region Imaging Spectrograph (IRIS; De Pontieu et al. 2014) and Hinode (Tsuneta et al. 2008). We focus on a quiet Sun area with small-scale structures in the transition region and investigate their relation with the underlying magnetic field.

In our study, we use IRIS level-2 spectra and slit-jaw-images. We analyse large dense raster with 400 steps and a step size of $0.35^{\prime \prime}$, a spatial scale along the slit of $0.17^{\prime \prime}$ pixel $^{-1}$, and a spectral resolution of $40 \mathrm{~m} \AA$. The raster covers a $140^{\prime \prime} \times 170^{\prime \prime}$ field-of-view. We focus on the Si Iv line at $1393.8 \AA$, which corresponds to the transition region emission at $\log T=4.9$, and the Lyman continuum of $\mathrm{Si}$ I that is formed near the temperature minimum. To investigate the temporal evolution of the small-scale structures we use IRIS slit-jaw-images at $1400 \AA$ (thereafter SJI1400) with a spatial scale of $0.17^{\prime \prime}$ pixel $^{-1}$, a cadence of $63.5 \mathrm{~s}$ and an exposure time of approximately $60 \mathrm{~s}$. The SJI1400 covers the $175^{\prime \prime} \times 175^{\prime \prime}$ field-of-view around the slit.

To investigate the evolution of the photospheric magnetic field, we use Hinode SOT data obtained in the FGSIV200 mode. We use the level-0 data: maps of the Stokes parameter I (intensity image) and V. We use them to create the line-of-sight magnetograms and the procedure is described in the next subsection. Hinode provides observations with $61.4^{\prime \prime} \times 81.9^{\prime \prime}$ field-of-view at a spatial scale of $0.16^{\prime \prime}$ per pixel and at a cadence of $35 \mathrm{~s}$. For more details about our regions-of-interest, we refer to Table 6.1. 
Table 6.1: Overview of the observations

\begin{tabular}{llccccc}
\hline \hline instrument & observable & start & end & exp. time $[\mathrm{s}]$ & $\mathrm{X}\left[{ }^{\prime \prime}\right]$ & $\mathrm{Y}\left[{ }^{\prime \prime}\right]$ \\
\hline Hinode SOT & Stokes V, I & $00: 21: 07(+1 \mathrm{~d})$ & $02: 58: 36(+1 \mathrm{~d})$ & 35 & -82 & -28 \\
IRIS SJI & 1400 ̊ & $23: 27: 28$ & $02: 59: 15(+1 \mathrm{~d})$ & 60 & -120 & -41 \\
IRIS raster & Si IV, continum & $23: 27: 28$ & $02: 59: 15(+1 \mathrm{~d})$ & 30 & -120 & -41 \\
\hline
\end{tabular}

\subsubsection{Data reduction}

\subsubsection{Hinode data}

For the Hinode data we apply the six-step preparation procedure that is described schematically in Fig. 6.1.

First, we convert the raw data to unsigned 16-bit integers. The next step is to produce level-1 data. For this, we use the Hinode reduction software which is available in SolarSof $t^{1}$. We want to create the magnetogram and to this end, the map of the Stokes parameter V is divided by the map of the Stokes parameter I. We obtain two magnetograms which correspond to the negative and positive part of the profile of the Stokes parameter $\mathrm{V}$ respectively, from which the average magnetogram is calculated. The data are used in our analysis were recorded by two parts of the CCD detector separately, therefore the data are merged to obtain the final magnetogram. In the next step, we select magnetograms that are closest in time to the slit position of the raster image. The selected magnetograms are co-aligned and rebinned to the IRIS SJI1400 resolution.

From this procedure, we obtain a time series of spatially aligned magnetograms at the same spatial scale as the IRIS SJI1400 images.

\subsubsection{IRIS slit-jaw images}

For the IRIS SJI level-2 data, we apply three-step procedure that is also shown schematically in Fig.6.1.

First, we define pairs of the Hinode magnetograms and almost simultaneous obtained slit-jaw-image as well as the corresponding IRIS raster maps. Then, slit-jaw-images are corrected for artifacts, such as dust spots, using the software which is available in the SolarSoft

The next and main step is the co-alignment of the SJI1400 images. This step is critical to the further analysis of the spatial and temporal evolution of small-scale structures. Therefore, we use a cross-correlation technique and the sub-pixel precision alignment.

Using this procedure, we obtain a dust-corrected and a spatially aligned series of SJI1400.

\subsubsection{IRIS raster map}

The Fig. 6.1 shows schematically the preparation procedure of the raster map. We use the level-2 data obtained by the FUVL camera.

\footnotetext{
${ }^{1}$ fg_prep.pro available at SolarSoft, http://darts.jaxa.jp/pub/solar/ssw/

2iris_dustbuster.pro available at SolarSoft, https://darts.isas.jaxa.jp/pub/ssw/.
} 


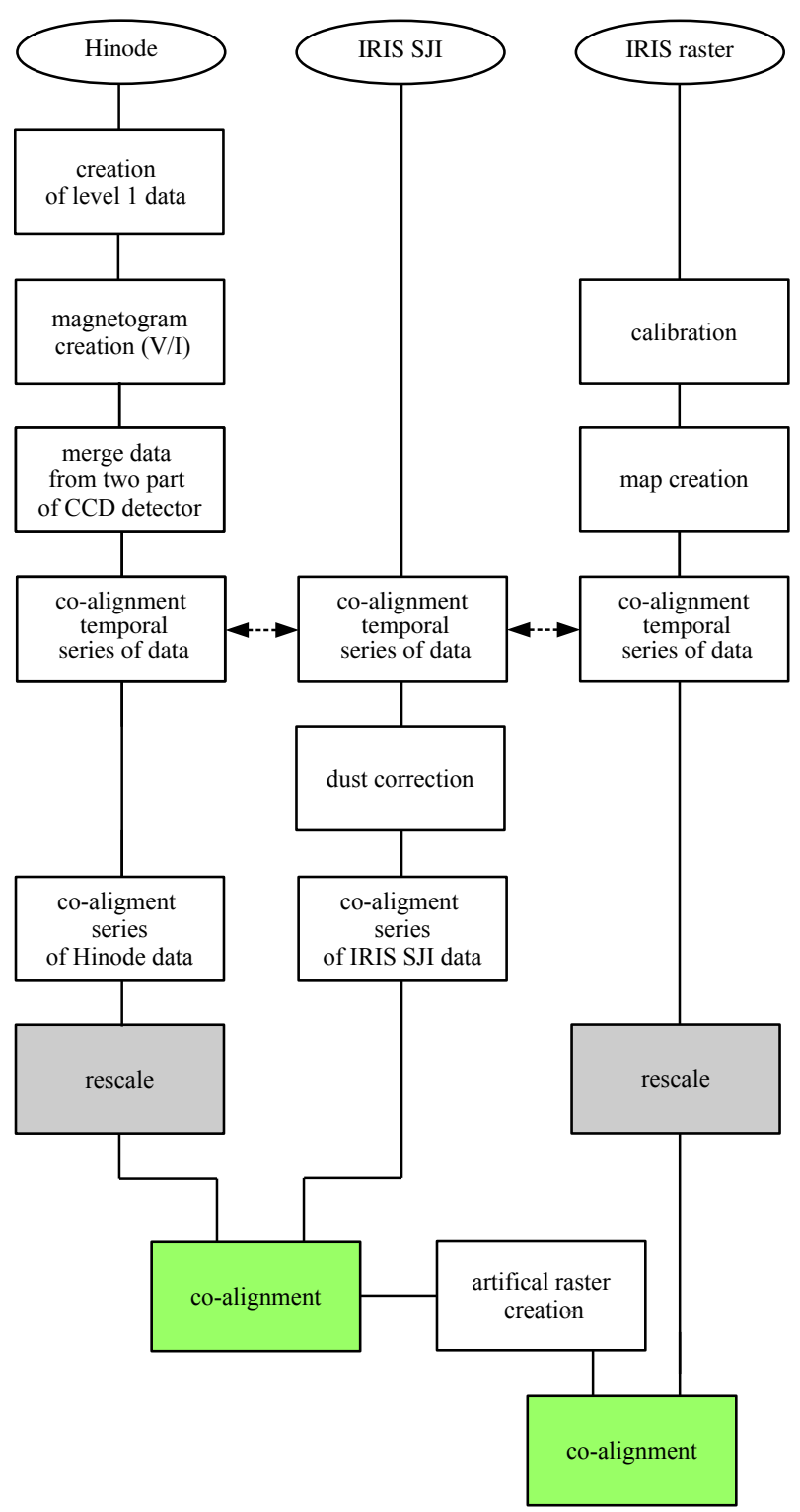

Figure 6.1: Flowchart of data preparation process. See Sect.6.2.2. 
In the first and main step, we calibrate the wavelength scale using the Fe II line at 1392.82 A as a reference.

Then, we prepared the intensity map of Si IV at $1393.76 \AA$. To this end, we integrated over the whole line covering a range of $0.76 \AA$ around the line center. In this analysis, the continuum is subtracted. We also created the continuum map. For this the continuum intensity is integrated from $1394.35 \AA$ to $1395.76 \AA$. For the density calculation, we prepared intensity maps of O IV lines at $1399.77 \AA$ and $1401.16 \AA$. We calculate the line intensity integrated around the lines center over a range of $0.6 \AA$, for this map we subtract the continuum emission. We fit a gaussian function to the Si Iv line profiles. The fitting parameters are used to create a map of the Doppler velocity (line center position) and a map of the full width at half maximum (FWHM, this is the Gaussian width multiplied by $2 \sqrt{\ln 2} \approx 1.67)$.

Finally, the raster maps are rescaled to the proper aspect ratio.

\subsection{Descriptions of structures}

Using the quiet Sun data we investigate the small-scale loop-like structure (Sec.6.3.1) and small-scale magnetic patches (Sec.6.3.2). The aim of this study is to analyse the spatial and temporal evolution of these structures as well as the relation with the underlying magnetic field.

\subsubsection{Small-scale loop-like structure}

The small-scale loop-like structures in the transition region were investigated before by Hansteen et al. (2014) using IRIS data. Their study does not provide information about the magnetic field and presents structures near the solar limb. In this study, we focus on small-scale loop-like structure located near the center of the solar disk and analyse the evolution of the associated magnetic field.

Using a slit-jaw-image, we identified, by eye, the small-scale loop-like feature in the full-field-of-view. Then, we defined the region-of-interest (thereafter ROI-1) of $9^{\prime \prime} \times 9^{\prime \prime}$, centered at $\left[-111^{\prime \prime},-34^{\prime \prime}\right]$, which includes this feature. We follow the structure. Using the raster maps, we recognise that the small-loop like structure is clearly visible in Si IV line (Fig 6.2 a). Therefore, we defined this structure by the enclosed contour line at a level of the half the maximum intensity in the Si Iv image (cf. red contour in Fig 6.2 a). The structure is not visible in the continuum map (Fig 6.2b).

To study the temporal and spatial evolution of small-scale loop like structure we analised a movie ${ }^{3}$ (Mov 1). It shows SJI1400 and corresponding magnetograms as well as the raster maps of $\mathrm{Si}$ IV and continuum. The movie presents ROI-1 from 01:41UT to 02:03 UT. In raster maps, the slit positions which at the time the SJI1400 are taken are marked. In Figure 6.3 we show the most interesting snapshots of this movie. They present the evolution of the small-scale loop-like structure in SJI400 (Fig 6.3a-e) and magnetograms (Fig 6.3 $\mathrm{f}-\mathrm{j})$.

The small-scale loop-like structure has a length of $5^{\prime \prime}$ with an approximately $3^{\prime \prime}$ long bright core which corresponds well to the contour from the Si IV raster map. The size of

\footnotetext{
${ }^{3}$ movie (Mov 1), http://www2 .mps.mpg.de/data/outgoing/barczynski/mov/mov1.mp4
} 


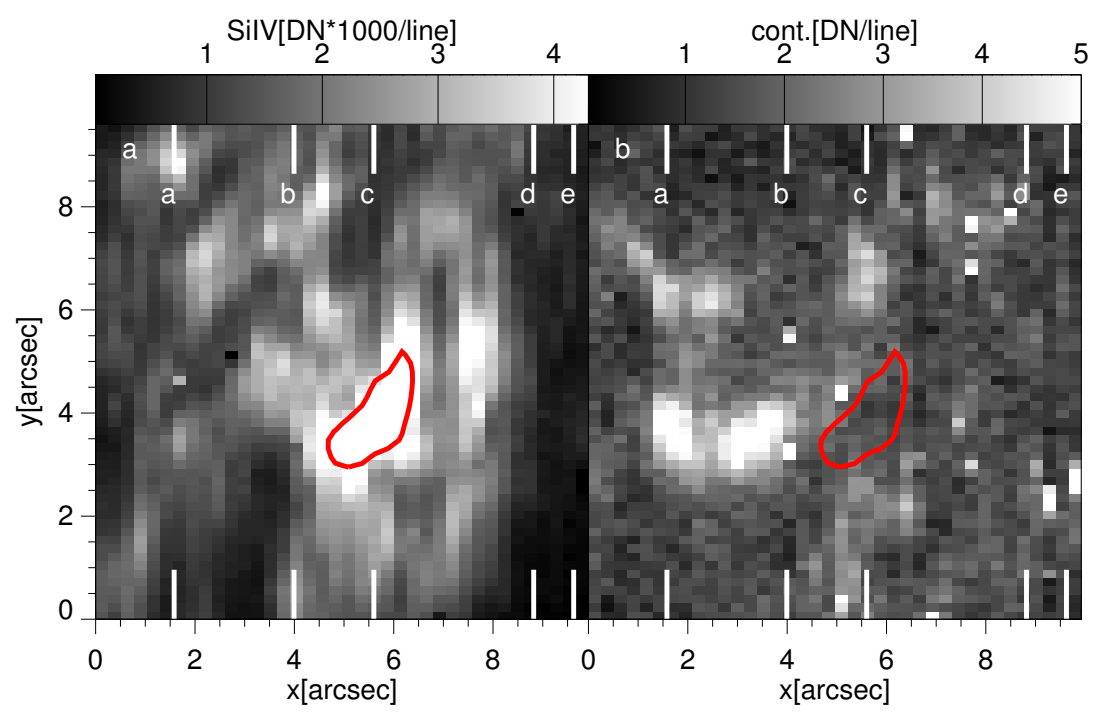

Figure 6.2: The small-scale loop-like structure observed in $\mathrm{Si}$ IV line (a) and continuum emission (b). The figure presents raster maps obtained from 01:43UT to 02:03UT. The red contour line, defined as half of maximum intensity of the Si Iv map, shows the smallscale loop-like structure. The white tick marks present the slit positions which temporary correspond with images in Fig 6.3 .

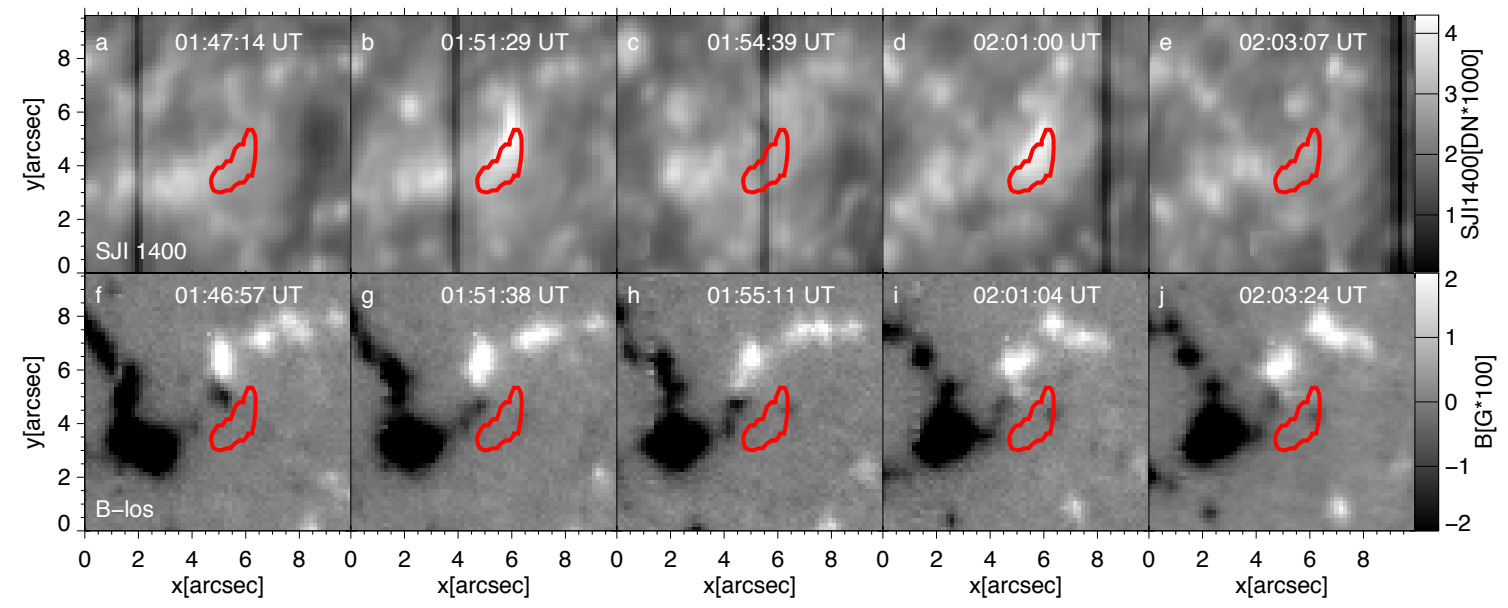

Figure 6.3: A sequence of images of the small-scale loop-like structure observed in SJI1400 (a-e) and the underlying magnetic field (f-j). The selected images present the most important phenomena in the region of interest between 01:47 UT and 02:03 UT. The red contour line are defined in Fig 6.2 a.

the structure is consistent with the small-scale loops observed by Winebarger et al.(2013) and Hansteen et al. (2014).

Using the SJI1400 we noticed that the small-scale loop-like structure appears between 01:50:28UT and 01:51:03UT (Fig6.3p), then disappears (Fig 6.3 ). It is visible again between 02:01:04UT and 02:02:49UT (Fig 6.3 d) and finally completely vanishes (Fig 6.3 e). For a deeper analysis of the temporal evolution of this feature, we study the brightness 
variability. To this end, we calculate the average brightness of the SJI1400 in the area inside the contour line, for each frame. The brightness variability is presented in Fig 6.4 b. For all measurements, the average intensity of the small-scale loop-like structure is at least two times larger than the median intensity of the individual frame. In this diagram, the increase of intensity is steeper than decrease, for both peaks. The life time of this feature (approximately 100 s) is comparable with small-scale loops observed in previous studies (Hansteen et al. 2014).

Comparing the slit-jaw-images (Fig6.3a-e) to the Hinode magnetograms (Fig 6.3 $\mathrm{f}$ $\mathrm{j}$ ), we found two opposite magnetic field concentrations that can be closely related to the footpoint of the small-scale loop-like structure. The configuration of the magnetic field does not change much during the observation time. The magnetic flux of ROI-1 (Fig 6.4a) change slightly with time. These changes are independent of the brightness variation (Fig 6.4p). The Pearson's correlation coefficient between the magnetic flux and intensity is nearly zero $(-0.06)$.

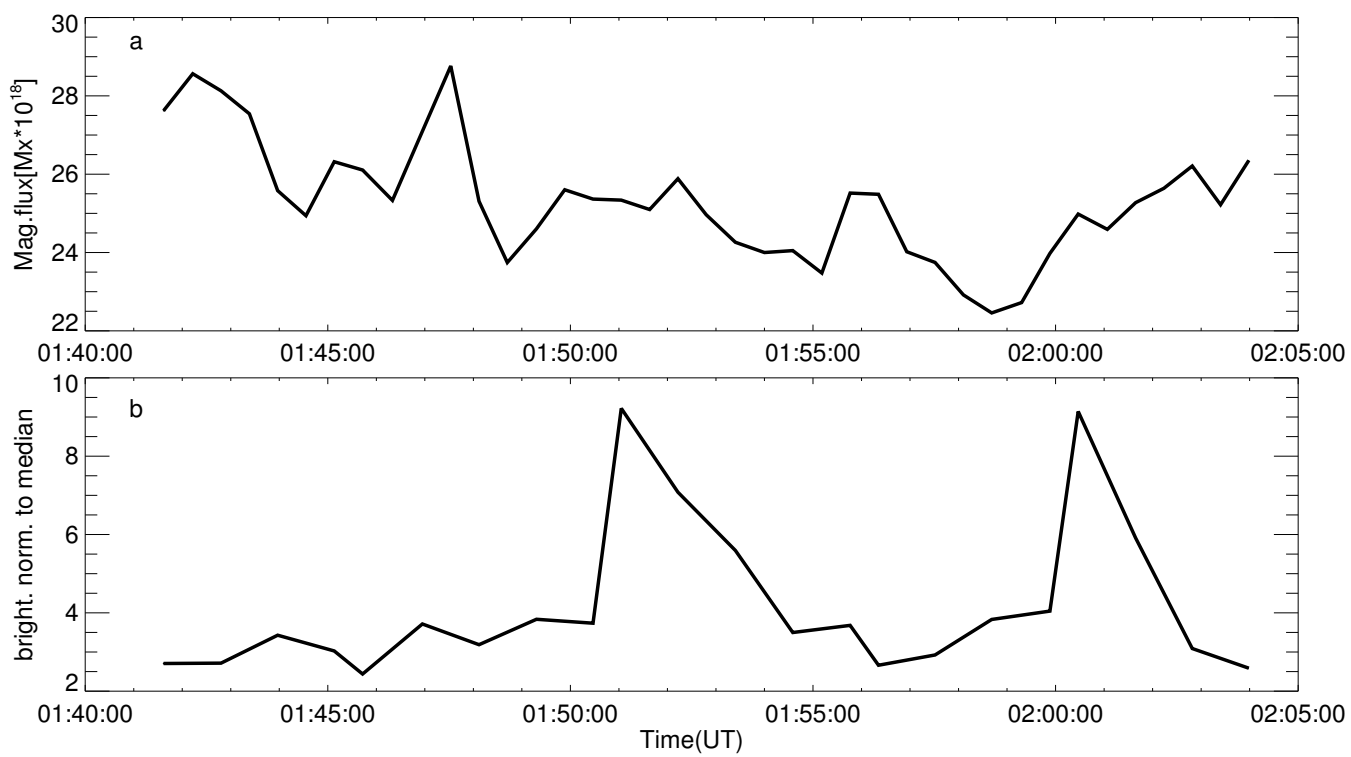

Figure 6.4: Temporal variation of the magnetic flux (a) and the brightness (b) of the small-scale loop-like structure. The magnetic flux is calculated for the whole ROI-1. The brightness in SJI1400 is computed as the average value of the area enclosed by the red contour lines (Fig 6.3 $\mathrm{a}-\mathrm{e}$ ).

We study the properties of the Si Iv line by fitting a single Gaussian. First, we discuss the velocity properties. The Si Iv Dopplergram (Fig 6.5a) shows that the southern leg of the feature is more strongly redshifted than the northern one. The study of individual spectra (Fig 6.6) confirms that effect, showing redshifts of $5 \mathrm{~km} \mathrm{~s}^{-1}$ for the northern leg and $16 \mathrm{~km} \mathrm{~s}^{-1}$ for the southern leg. The Gaussian fits also give information on the line width. Here, we consider the FWHM for the line width and plot the results in Fig 6.5b. The FWHM is larger $\left(\sim 45 \mathrm{~km} \mathrm{~s}^{-1}\right)$ in the southern and the central part of the structure than in the northern part $\left(\sim 35 \mathrm{~km} \mathrm{~s}^{-1}\right)$. These results are higher than the FWHM measure in the Si IV at $1403 \AA$ for the network (Peter 2001). 


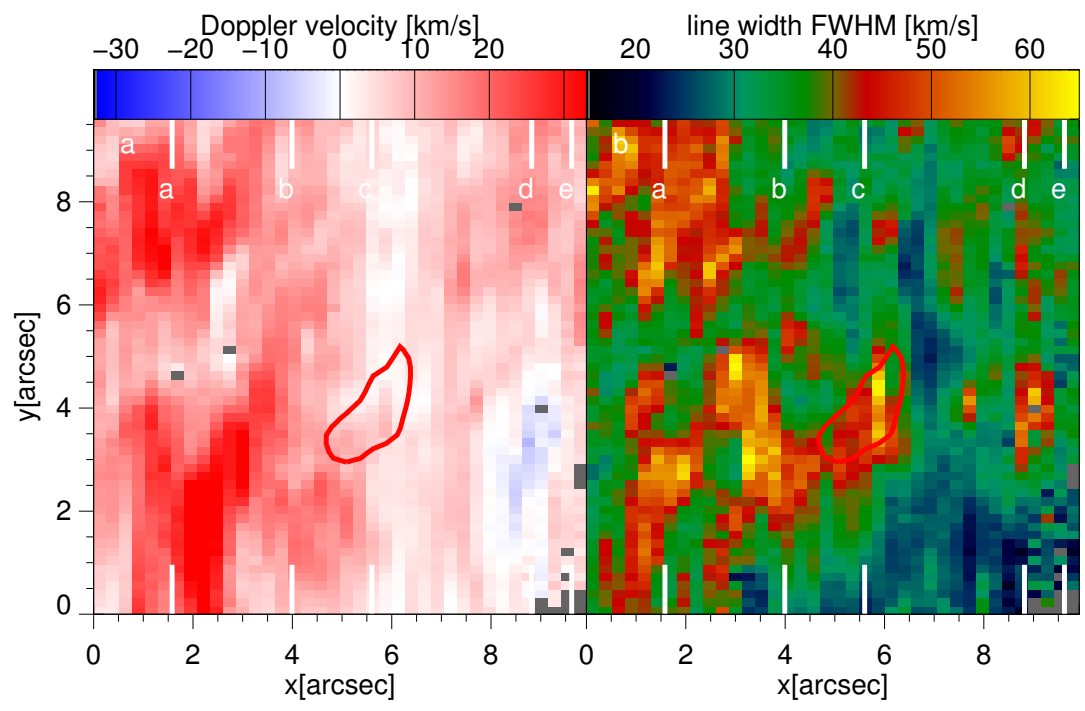

Figure 6.5: The Doppler (a) and line width FWHM (b) map of the region with small-scale loop-like structures. The red contour line and the white thick mark the same as in Fig 6.2a.
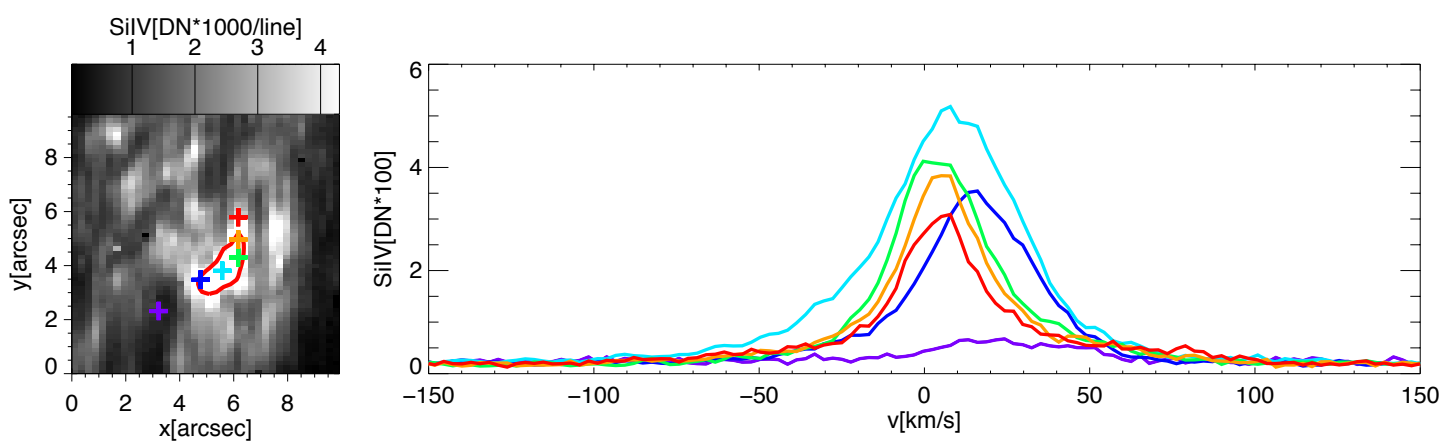

Figure 6.6: The raster map of $\mathrm{Si}$ IV and selected spectra. The raster map the same as in Fig 6.2 a. The color-coded plus signs in the raster map (a) correspond to the spectral lines (b). The South footpoint (dark blue sign plus) has a higher redshift $\left(16 \mathrm{~km} \mathrm{~s}^{-1}\right)$ than the North footpoint (red sign plus, $5 \mathrm{~km} \mathrm{~s}^{-1}$ ). The spectra cover the whole small-loop like-structure event.

Using SolarSof ${ }^{4}$ we calculated the average density of $10^{10.92} \mathrm{~cm}^{-3}$ of the small-loop like-structure of area enclosed by the red contour line. It is significantly higher than the density in network $\left(10^{9} \mathrm{~cm}^{-3}\right)$ and internetwork $\left(4 \times 10^{9} \mathrm{~cm}^{-3}\right)$ area of the quiet Sun measured by Innes (2001) with the same $\mathrm{O}$ Iv lines ratio as in this study.

These results imply that the small-scale loop-like structure has a lifetime and size comparable with the small-scale loop observed in the transition region. The variability of this structure observed in SJI1400 is independent on the magnetic flux changes. The physical properties of this structure, such as FWHM and density are significantly different compared with the network and internetwork area observed in the transition region line.

\footnotetext{
${ }^{4}$ iris_ne_oiv.pro available at SolarSoft, https://darts.isas.jaxa.jp/pub/ssw/
} 
In Sect. 6.4 we present a more detailed discussion of the properties of the small-scale loop-like structure.

\subsubsection{Two magnetic field patches}

Using simultaneous observations of the magnetic field and the EUV emission (Fig 6.7), we study two magnetic field concentrations that look similar in the magnetogram, but they have a different response in the EUV images. We analyse the dynamics of these structures in the EUV images and the evolution of the underlying magnetic field.

Based on the Hinode magnetograms of the full field-of-view, we identify, be eye, two similar magnetic field patches. Then we define a $14^{\prime \prime} \times 16^{\prime \prime}$ region-of-interest (thereafter, ROI-2), centered at $\left[-102^{\prime \prime}, 15^{\prime \prime}\right]$, which cover these structures. Similarly to Sec. 6.3.1, we analysed a movie $5^{5}$ (Mov2) that shows the temporal evolution from 01:36 UT to 02:58 UT in magnetograms and SJI1400, as well as raster maps of Si IV, continuum intensity, Doppler velocity, FWHM, raster-reconstructed map of the magnetic field and raster-reconstructed map of SJI1400 intensity. The procedure of creation of the rasterreconstructed map is described in Sect.7.2.2.2.

Using the raster-reconstructed map of the magnetic field (Fig 6.7]), we defined the magnetic field patch as the feature that is enclosed by the contour line at $|\mathrm{B}|>40 \mathrm{G}$. This threshold was chosen arbitrarily and is used to define patches localization in the corresponding images, for which these contours were applied. In ROI-2, we found five structures with $|\mathrm{B}|>40 \mathrm{G}$. In our analysis, we focus only on two of them (N and $\mathrm{S})$, which are most distinct and similar.

Based on the Hinode magnetograms (Fig 6.7e-h), we investigate the evolution of the magnetic field patches. Structures $\mathrm{N}$ and $\mathrm{S}$ are created by merging unipolar, highly dynamic, small-scale magnetic field concentrations (thereafter, called precursor), as seen in Fig. 6.77. They have an ellipsoidal shape and a length around $1 \mathrm{Mm}$. Four precursors merge together and create the structure $\mathrm{N}$, three precursors form structure $\mathrm{S}$. Two independent and compact structures ( $\mathrm{N}$ and $\mathrm{S}$ ) appear at 01:55 UT. The further evolution (Fig.6.7f-h) of patches is significantly slower. These structures keep the same size almost to the end of the observation series at 02:58 UT (Fig.6.7h). In general, they have a size less than $1.5 \mathrm{Mm}$ and a circular shape, which slowly evolves to be elliptical in the final stage of the observation.

Using the IRIS1400 (Fig 6.7 $\mathrm{a}-\mathrm{d}$ ) we analyse the temporal evolution of these structures. The analysis of the temporal and spatial evolution of the structure $\mathrm{N}$ presents a clear relation between the concentration of intensity in the SJI1400 and the concentration of the underlying magnetic field. This trend exists even for precursors (compare image Fig 6.7 a and Fig 6.7 ). In contrast, we do not recognize any SJI1400 intensity which corresponds to the magnetic field patch $S$.

For an investigation of how small-scale magnetic field patches are related to the SJI1400 intensity, we study the temporal evolution of the average magnetic flux and average intensity which are calculated of $1.6^{\prime \prime}$ around the magnetic patches (Fig 6.7 $\mathrm{d}$ and $\mathrm{h}$ ), for each frame. The temporal evolution of the magnetic field and intensity is presented in Fig6.8. We do not find a direct relation between the magnetic flux and intensity for

\footnotetext{
${ }^{5}$ movie (Mov 2), http://www2 .mps.mpg.de/data/outgoing/barczynski/mov/mov2 .mp4
} 
6 The small-scale structure in the transition region

both structures ( $\mathrm{Tab} 6.2$ ). The changes of the magnetic fluxes of the $\mathrm{N}$ and $\mathrm{S}$ structures are highly correlated $(\mathrm{Tab} 6.2)$. In contrast, their intensity changes present a weak anticorrelation.

Table 6.2: The Pearson's correlation coefficient of the magnetic flux and intensity of the small-scale magnetic field patches.

\begin{tabular}{lc}
\hline \hline relation & correlation coeff. \\
\hline magnetic flux N vs. S & 0.87 \\
int. SJI1400 N vs. S & -0.20 \\
magnetic flux vs. int. SJI1400, N & 0.15 \\
magnetic flux vs. int. SJI1400, S & -0.62 \\
\hline
\end{tabular}

To compare the magnetic field patches to the emission from the temperature minimum and the transition region we study the continuum and $\mathrm{Si}$ IV raster maps, respectively. The continuum raster map presents a clear emission from structure $\mathrm{N}$. We do not recognize the structure $S$ in this image. The Si IV raster map does not show any emission associated with the $\mathrm{S}$ structure and only a weak signal at the $\mathrm{N}$ structure.

Using the velocity map (Fig 6.7k) created in the Si IV line we study the plasma motion in the line-of-sight direction. A rough inspection of Doppler velocity shows redshifts at approximately $10 \mathrm{~km} \mathrm{~s}^{-1}$, for both structures. This value is almost the same as the velocity of the surrounding area of ROI-2. The average spectra of structures $\mathrm{N}$ and $\mathrm{S}$ (Fig $6.7 \mathrm{~m}, \mathrm{n}$ ) show redshifts of $15 \mathrm{~km} \mathrm{~s}^{-1}$ and $10 \mathrm{~km} \mathrm{~s}^{-1}$, respectively. Based on the raster map of the FWHM (see Mov 2) we obtain the same average FWHM of $39 \mathrm{~km} \mathrm{~s}^{-1}$ for both structures.

To emphasize the differences between structures $\mathrm{N}$ and $\mathrm{S}$ we present the average values of several physical quantities in Tab 6.3. These values are calculated from the area enclosed by the yellow contour lines in Fig 6.7 $\mathrm{a}-1$. The magnetic flux and intensity of SJI1400 are calculated from the single images obtained at 02:03 UT. The density is computed in the same manner as in Sec.6.3.1. Both structures have almost the same average magnetic field strength, however the magnetic flux in structure $\mathrm{N}$ is $42 \%$ larger than for structure S. The structure N has a higher intensity than structure S in SJI1400 (5 times), continuum (3 times) and Si IV (2 times) raster map. The electron density of structure $\mathrm{S}$ is significantly higher than for structure $\mathrm{N}$.

\subsection{Discussion}

\subsubsection{Small-scale loop-like structure}

In this chapter, we studied the temporal and spatial evolution of small-scale loop-like structures. We found a structure which has a similar life-time and size as the small-scale loops investigated by Winebarger et al. (2013) and Hansteen et al. (2014).

The SJI1400 and magnetogram show that small-scale loop-like structure connects two footpoints which are rooted in areas of the opposite magnetic field polarity. The relation of this structure with the photospheric magnetic field is difficult to define. There is a lack of clear evidence for flux cancellation and emergence based on the photospheric magnetic field observations. 
Table 6.3: Comparison of the magnetic field patches.

\begin{tabular}{|c|c|c|c|}
\hline & $\begin{array}{c}\mathrm{N} \\
\text { northern feature }\end{array}$ & $\begin{array}{c}\text { ratio } \\
\frac{N}{S},\left(\frac{|N-S|}{|N+S|}\right)\end{array}$ & $\begin{array}{c}\text { S } \\
\text { southern feature }\end{array}$ \\
\hline$\overline{\mathrm{B}[\mathrm{G}]}$ & 127 & $1.09(0.04)$ & 117 \\
\hline mag. flux $\left[\mathrm{Mx} 10^{14}\right]$ & 1.96 & $1.38(0.16)$ & 1.43 \\
\hline int. SJI1400 [DN] & 1510 & $4.94(0.66)$ & 306 \\
\hline int. cont. [DN/line] & 5.4 & $3.28(0.53)$ & 1.6 \\
\hline int. Si IV [DN/line] & 921 & $2.35(0.40)$ & 391 \\
\hline $\log \left(\right.$ density $\left./ \mathrm{cm}^{-3}\right)$ & 11.2 & - & $>13$ \\
\hline Doppler vel. $\left[\mathrm{km} \mathrm{s}^{-1}\right]$ & 15 & $1.5(0.2)$ & 10 \\
\hline FWHM $\left[\mathrm{km} \mathrm{s}^{-1}\right]$ & 39 & $1.0(0.0)$ & 39 \\
\hline
\end{tabular}

We suggest that the magnetic field plays an important role in the creation and evolution of this structure, and in the following we present one possible scenario. The lack of emission in the temperature minimum and high emission in the $\mathrm{Si}$ IV suggests that first the plasma emerge and then the reconnection take place in the upper chromosphere or transition region. We analyse the temporal evolution of the structure in SJI1400 which covered the $\mathrm{Si}$ IV line. These images present not only the transition region emission but also cooler plasma in the chromosphere emission. In this case we suggest that the unsolved fine structures observed in the previous works had not only a transition region temperature. The two peaks of intensity observed in SJI1400 suggest that scenario of the plasma emergence and reconnection take place two times during $10 \mathrm{~min}$. One can assume that the stable configuration of the magnetic field allows for the repetition of this scenario in one location for several times. We suggest that the long lived "nest" of loops observed by Hansteen et al. (2014) is an example of this phenomenon.

We found shifts in the Doppler velocities which change from the northern footpoint $\left(16 \mathrm{~km} \mathrm{~s}^{-1}\right)$ to the southern footpoint $\left(5 \mathrm{~km} \mathrm{~s}^{-1}\right)$. This suggest a draining flow and we do not see a siphon flow pattern on the velocity map. These values are comparable with the mean ascent velocity of the small-scale structure observed in the chromosphere by Martínez González et al.(2010). To further discuss the velocity properties of this structure the calculation of the Doppler shift from different temperatures in the solar atmosphere is needed.

The average density of the small-scale loop-like structure $\left(10^{10.92} \mathrm{~cm}^{-3}\right)$ is higher than density of the small loop $\left(10^{9} \mathrm{~cm}^{-3}\right)$ investigated by Winebarger et al. (2013) and higher than density of the plasma in the network and internetwork of the quiet Sun measured in the $\mathrm{O}$ IV lines.

\subsubsection{Two magnetic field patches}

In this study, we discuss the properties of two magnetic field patches. They have similar geometrical properties such as circular shape and a size of approximately $1.5 \mathrm{Mm}$. Both structures have a lifetime of $1 \mathrm{~h}$ and an average magnetic field of approximately of $120 \mathrm{G}$.

The study of the temporal evolution of the magnetic field shows that several smallscale magnetic field concentrations merge and create the magnetic field patch. Simultaneously, the continuum map and the SJI1400 show only emission from one structure 
$(\mathrm{N})$ and a lack of the emission associated with the other structure (S). Additionally, these structures are not visible in the raster map of Si IV. This implies that the continuum intensity has the main contribution in the SJI1400 of ROI-2. Based on the above information we suggest that these two magnetic field patches are generated by a convective collapse (Spruit 1979). In this scenario, initially the magnetic flux is almost uniformly distributed over a large area. The convection motion make the plasma strongly moving downwards in one place. This causes that plasma start to be squeezed, therefore the magnetic field start to be more concentrated. In the same place the magnetic pressure will be in equilibrium with gas pressure and further the magnetic field start inhibiting the heating of the material. Then the magnetic field will be squeezed more and will create a strong magnetic field concentration. One can assume this process is responsible for creation of the precursors and the magnetic patches. According to this scenario, in the solar atmosphere the layer of the $\tau=1$ is going down, and this is consistent with the bright emission from this structure observed in the UV continuum. The large plasma density (more than $10^{11} \mathrm{~cm}^{-3}$ ) also agrees with the convective collapse scenario.

\subsubsection{Conclusion}

We investigated the small-scale loop-like structures and two magnetic patches observed in a quiet Sun area of the transition region. We focus on the temporal and spatial evolution of these structures as well as their connection to the underlying magnetic field.

The analysis of the small-loop like structure (ROI-1) suggests that this structure is created by the magnetic field which emerges from the lower atmosphere. The reconnection takes place in the chromosphere or transition region causes emission that is observed in Si IV. The reconnection occurs two times during 10 minutes, in this time the magnetic field configuration remains almost unchanged. This implies that large scale magnetic field can play an important role in stabilization of the small-scale magnetic field configuration.

The discussion suggests that two magnetic field patches (ROI-2) are probably examples of the convective collapse. These structures have a similar magnetic field density and magnetic field flux, also similar temporal and spatial evolution, but different EUV emission associated with them. We suggest that these structures have a different inclination, therefore the EUV emission in the line-of-sight is different. The similar evolution of the magnetic field of these structures (of approximately 1 hour) implies that the largescale magnetic field structures also play an important role in evolution these small-scale magnetic patches. 

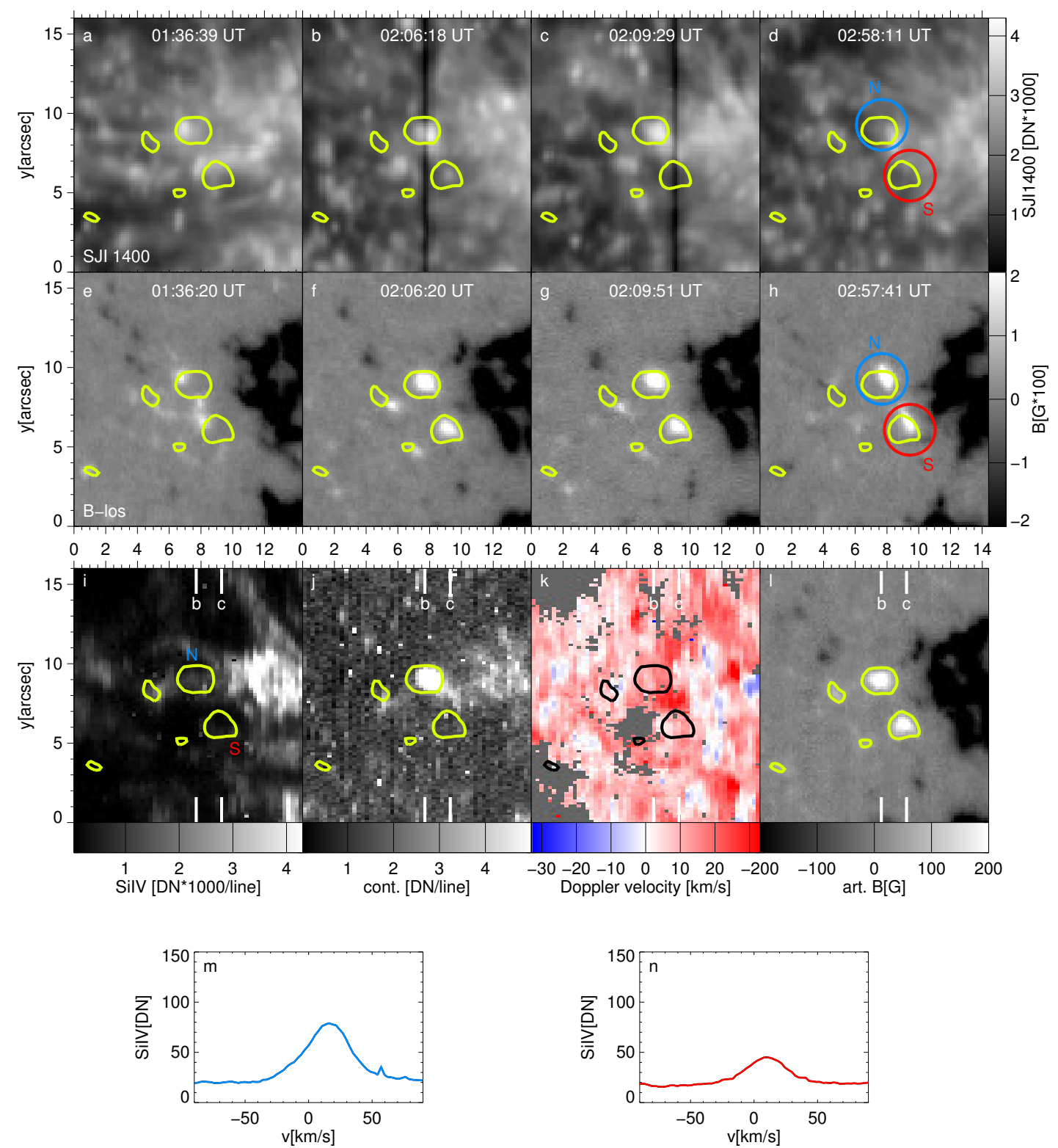

Figure 6.7: The two magnetic field patches in the different observable. The temporal evolution of the magnetic patches (a-d) and the corresponding emission in SJI1400 (e-h) from 01:36 UT to 02:58 UT. The blue and red circles defined the area of enclosed North and South magnetic field patches respectively. The average profile computed from the North area (m) present a Gaussian shape (n) with redshift of $15 \mathrm{~km} \mathrm{~s}^{-1}$, the South structure has a Gaussian shape with redshift of $10 \mathrm{~km} \mathrm{~s}^{-1}$. The raster image of Si IV (i) do not shows any clear patter in the place which corresponds to the magnetic patches. The continuum emission (j) in the North structure is significantly brighter than background, the South structure is not visible. The Doppler map (k) shows that both structures are redshifted. The raster reconstructed map of the magnetic field (l) shows clearly two magnetic field patches. The contour at the threshold of $40 \mathrm{G}$ from this map is used to identify the structures on the rest of images (a-k). 


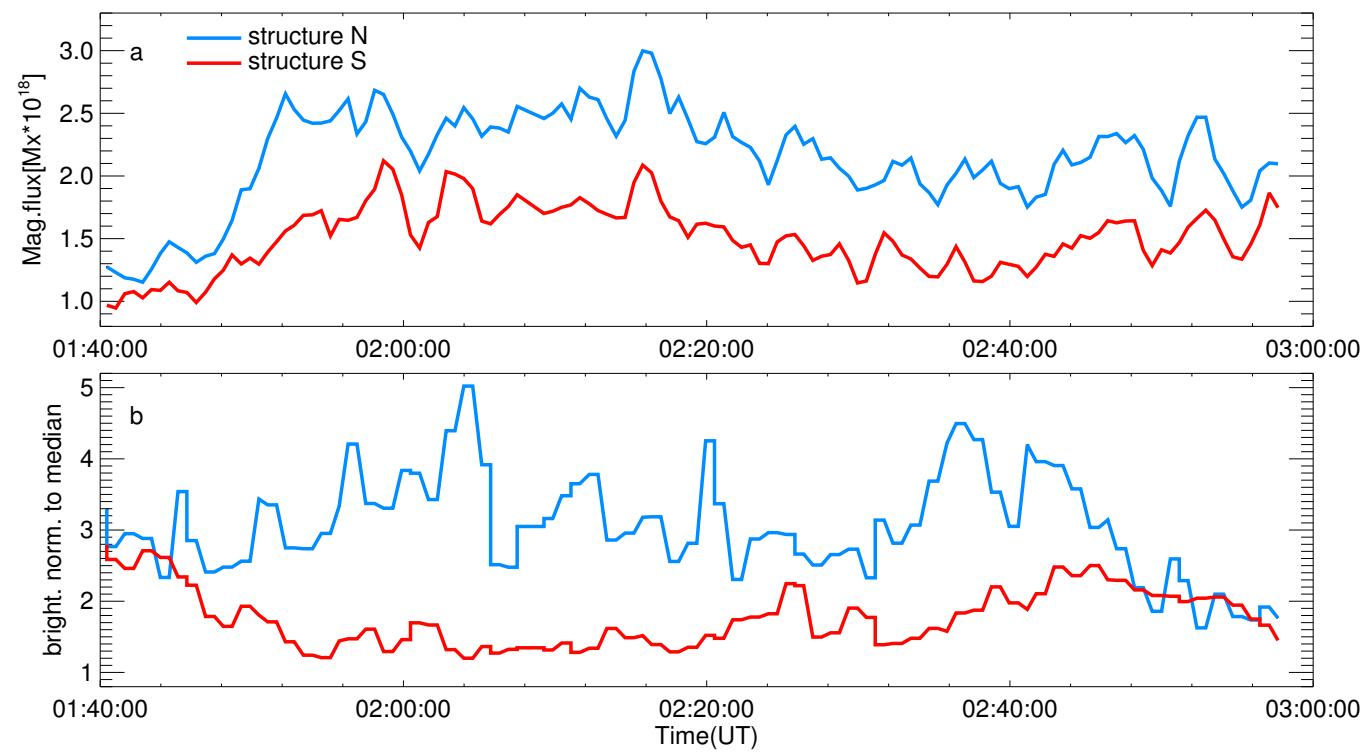

Figure 6.8: Variability of the magnetic flux of the two magnetic field patches and correspond to them brightness in SJI1400. The magnetic flux is computed from areas enclosed by the red and blue circles for the North and the South structure respectively (Fig 6.7 d). The average brightness of is calculated in this same manner (Fig 6.7h). On the diagram we see short and long term trend. The changes of the magnetic field of the North and South structures are similar. 


\section{Emission of solar chromospheric and transition region features related to the underlying magnetic field}

*This chapter is based on a journal article that is in preparation.

Context: The emission of the upper atmosphere of the Sun is closely related to magnetic field concentrations at the solar surface.

Aims: It is well established that this relation between chromospheric emission and magnetic field is nonlinear. Here we investigate systematically how this relation, characterised by the exponent of a power-law fit, changes through the atmosphere, from the upper photosphere through the temperature minimum region and chromosphere to the transition region.

Methods: We use spectral maps from the Interface Region Imaging Spectrograph (IRIS) covering $\mathrm{Mg}$ II and its wings, $\mathrm{C}$ II, and Si IV together with magnetograms and UV continuum images from the Solar Dynamics Observatory. After a careful alignment of the data we determine the indices (or exponents) by assuming a power-law dependency between each pair of observables, and do this for different spatial resolutions.

Results: While the correlation between emission and magnetic field drops monotonically with temperature, the power-law index shows a hockey-stick-type variation: from the upper photosphere to the temperature-minimum it drops sharply and then increases through the chromosphere into the transition region. This is even seen through the features of the $\mathrm{Mg}$ II line, i.e. from $\mathrm{k} 1$ to $\mathrm{k} 2$ and $\mathrm{k} 3$. It is irrespective of spatial resolution or weather we check regions of active region, plage, quiet Sun or coronal holes.

Conclusions: The relations between the emission and the magnetic field below and above the temperature minimum are governed by different mechanisms: Below the geometric effect of expanding flux tubes dominates because at high magnetic field strengths these quickly fill the available chromospheric volume. Above the temperature minimum however, the increasing sensitivity of the emission on the plasma heating is most relevant. 
7 Emission of solar chromospheric and transition region features related to the underlying magnetic field

\subsection{Introduction}

One of the major proxies to characterise the magnetic activity of a (cool) star is its chromospheric emission (e.g. Hall 2008, Reiners 2012). In particular the $\mathrm{Ca}$ II $\mathrm{H}$ and $\mathrm{K}$ lines in the ultraviolet (UV) at $3968 \AA$ and $3934 \AA$ and the $\mathrm{Mg}$ II $\mathrm{h}$ and $\mathrm{k}$ lines further in the UV near $2804 \AA$ and $2796 \AA$ are used. Using an index based on the $\mathrm{Ca}$ II lines a long-term monitoring program was set up at the Mount Wilson Observatory to investigate stellar activity cycles (Wilson 1978, Baliunas et al. 1995). To interpret the chromospheric indices in terms of magnetic activity and cyclic variation solar observations are a key. This is because only on the Sun can we directly study the spatio-temporal relation between chromospheric emission and the underlying magnetic field. The Interface Region Imaging Spectrograph (IRIS; De Pontieu et al. 2014) provides new solar data to this study of the spatial relationship of the chromospheric emission and the magnetic field through spectral maps in the Mg II lines. And IRIS adds information from the photosphere (through the wings of $\mathrm{Mg}$ II) and in particular from the transition region (from the doublets of $\mathrm{C}$ II and Si IV around $1335 \AA$ and $1400 \AA$ ).

Investigations of the relation of chromospheric emission and the (unsigned) photospheric magnetic field $|B|$ on the Sun date back more than half a century and are mostly based on studies of the $\mathrm{H}$ and $\mathrm{K}$ lines of $\mathrm{Ca}$ II. Comparing spectroheliograms and magnetograms in plage areas Leighton (1959) found a spatial correspondence between $|B|$ and $\mathrm{Ca}$ II. The first quantitative studies for the quiet Sun revealed a linear dependance (Skumanich et al. 1975, Nindos and Zirin 1998). However, Schrijver et al. (1989) suggested a power-law relation for the plage area, with an exponent of about 0.6, i.e. with a much weaker dependence of the chromospheric emission on the magnetic field. Likewise, later studies argued that a power-law (with an exponent less than 1) is found in plage and quiet Sun network regions (e.g. Harvey and White 1999, Ortiz and Rast 2005, Rezaei et al. 2007). A more detailed analysis of the quiet Sun was done by Loukitcheva et al. (2009). They compared magnetograms and Ca II K filtergrams separately for the network and internetwork regions of the quiet Sun. While for the network they found the well established power-law relation (exponent of about 0.5 ), in the internetwork the $\mathrm{Ca}$ II $\mathrm{K}$-line emission is independent of the underlying magnetic field. This is consistent with the general idea that the internetwork is not dominated by magnetic fields. More detailed information about power-law indices obtained in the previous studies is presented in Table 7.2 .

While there is a clear relation between the magnetic field and the chromospheric emission, even for the lowest magnetic field values the emission is not zero. The minimum energy flux through radiation from the chromosphere (and higher layers in the atmosphere) in the absence of magnetic field is termed basal flux (Schrijver et al. 1989, Schrijver 1992). This basal flux found in the non-magnetic regions is generally thought to be due to acoustic heating (e.g. Rutten and Uitenbroek 1991). Consequently, when searching for a physical connection between the surface magnetic field and the chromospheric emission one has to subtract this basal flux from the radiation from the chromosphere (or from higher/hotter regions).

A direct quantitative comparison of results from previous observational studies on the relation of the magnetic field to the chromospheric emission is difficult. Naturally the authors used different instrumentation, e.g. with different sensitivity with respect to the magnetic field or various widths of the spectral bandpass (or resolution) for the 
chromospheric emission. However, the common property of all these observations is a power-law relation between magnetic field and chromospheric emission with a power law index below unity.

This relation has been interpreted already by Schrijver et al. (1989) as being due to the geometry of the magnetic field expanding from the concentrations in the photosphere into the chromosphere. A higher (average) magnetic field strength corresponds to a denser packing of the (wine-glass shaped) magnetic flux tubes. Once these expanding tubes meet in the chromosphere (where Ca II forms) even a denser packing of the flux tubes cannot increase the chromospheric emission. Basically with increasing magnetic field the chromospheric emission saturates. Instead of a linear relation between field and emission the relation gets flatter for higher field strength, corresponding to a relation with a powerlaw exponent smaller than 1 . This idea was later confirmed by a proper two-dimensional model (Solanki et al.|1991).

When comparing the magnetic field and the emission from hotter regions of the upper atmosphere, the picture changes. For example (Pevtsov et al. 2003) found the powerlaw exponent to be (slightly) larger than one, and when considering the whole solar disk averages they found values being as high as almost two. We do not further discuss the implications of these results on coronal heating here, but highlight the question on how the relation of the magnetic field to the emission changes through the different temperature regimes of the atmosphere. In this study we will concentrate on the change from the upper photosphere through the temperature minimum region and the chromosphere to the transition region. This will then provide a test to the geometric picture explaining the relation of magnetic field to emission by investigating how this relation might change with temperature based on the model idea.

In this study we will use the term mag-flux relations for the relation of the magnetic field in the photosphere to the radiative flux from the different regions of the atmosphere. In addition to this we will also investigate the mutual relations of the radiative fluxes and term these flux-flux relations (in accordance with the existing literature). The flux-flux relations have been investigated extensively in the framework of stellar studies, simply because of the frequent lack of knowledge about the surface magnetic field of the stars under consideration (e.g. Ayres et al. 1981, Oranje 1986). Here we investigate the fluxflux relations mainly because we can analyse emission features from different temperature regimes observed with the same spectrograph simultaneously through the same slit. Thus any problems of spatial misalignment can be ruled out.

To get a good continuous temperature coverage we use data from IRIS (cf. Sect7.2.1). In particular the $\mathrm{Mg}$ II lines provide good diagnostics throughout the chromosphere (Leenaarts et al. 2013a b, Pereira et al. 2013). Using the wings of Mg II we add information from the upper photosphere, and the $\mathrm{C}$ II and $\mathrm{Si}$ IV provide details on the transition region. We complement these data with magnetograms from the Helioseismic and Magnetic Imager (HMI; Scherrer et al.2012) to study the mag-flux relations.

After a discussion on the preparation of the data and the analysis method (Sects.7.2 and 7.3) we show in Sect.7.4 that there is a continuous variation of the power-law exponent of the mag-flux relation, all the way from the photosphere into the transition region. Based on these results we discuss in Sect.7.5 the drop of the power-law index from the photosphere to the chromosphere in terms of the geometric model of Schrijver et al. (1989) and the rise of the same index into the transition region by the increasing sensitivity 
of the emission to the underlying magnetic field.

\subsection{Observations and preparation of data}

\subsubsection{Observations}

In this work we concentrate on observations acquired simultaneously by the Interface Region Imaging Spectrograph (IRIS, De Pontieu et al. 2014) and the Solar Dynamics Observatory (SDO, Pesnell et al.2012). We focus on the active region AR11850 with an extended plage area and compare the derived properties with data sets covering a quiet Sun region and an (on-disk) coronal hole (cf. Table 7.1).

In our study we use IRIS data covering the chromosphere and transition region using simultaneously recorded spectra and slit-jaw-images. The large dense rasters from IRIS cover 400 steps with a step size of $0.35^{\prime \prime}$ providing maps of the spectral line properties with a full field-of-view of about $140^{\prime \prime} \times 170^{\prime \prime}$. The spatial scale along the slit is about $0.17^{\prime \prime} /$ pixel. Here we concentrate on $\mathrm{Mg}$ II $\mathrm{k}, \mathrm{C}$ II and $\mathrm{Si}$ IV. While the different features of the $\mathrm{Mg}$ II profile, i.e., the $\mathrm{k} 3$ self-reversal, the $\mathrm{k} 2 \mathrm{r}$ peak and the $\mathrm{k} 1 \mathrm{r}$ minimum, originate from the upper to the lower chromosphere, C II and Si IV stem from the transition region. Details of the emission line properties are summarised in Table 7.3. The temperature of lines' formation should be taken with a grain of salt because these would apply only under equilibrium conditions. On the real Sun the atmosphere is quite dynamic and the traditional view of a stable stratified atmosphere certainly does not apply. Still the formation temperatures Table 7.3 provide some ordering of the lines (and their spectral features) with temperature in the upper solar atmosphere, in an average sense.

To investigate the response of the upper atmosphere to the photospheric magnetic field we use line-of-sight magnetograms from the Helioseismic and Magnetic Imager (SDO/HMI; Scherrer et al. 2012). These provide information on the full solar disk with a plate scale of $0.5^{\prime \prime} /$ pixel at a cadence of $45 \mathrm{~s}$. For a reliable alignment between the IRIS raster maps and the HMI magnetograms (Sect.77.2.2.2) and to investigate the temperature minimum region we employ the $1600 \AA$ channel of the Atmospheric Imaging Assembly (SDO/AIA; Lemen et al. 2012). The $1600 \AA$ images of AIA provide data of the full solar disk with a $0.6^{\prime \prime} /$ pixel plate scale at a temporal cadence of $24 \mathrm{~s}$. 
Table 7.1: IRIS data sets used in this study.

\begin{tabular}{lcclcccr}
\hline \hline & date & start (UT) & end (UT) & exposure time & raster type $\mathrm{e}^{\sqrt{\square}}$ & $\mathrm{X}^{\square}$ & $\mathrm{Y}^{\square}$ \\
\hline active region (AR 11850) & 24.09 .2013 & $11: 44: 43$ & $12: 04: 10$ & $2 \mathrm{~s}$ & & $-265^{\prime \prime}$ & $+88^{\prime \prime}$ \\
quiet Sun (QS) & 13.10 .2013 & $23: 27: 28$ & $02: 59: 15(+1 \mathrm{~d})$ & $30 \mathrm{~s}$ & $400 \times 0.35^{\prime \prime}$ & $-120^{\prime \prime}$ & $-41^{\prime \prime}$ \\
coronal hole (CH) & 12.10 .2013 & $12: 20: 28$ & $15: 52: 15$ & $30 \mathrm{~s}$ & & $+314^{\prime \prime}$ & $-140^{\prime \prime}$ \\
\hline
\end{tabular}

a In all cases large dense rasters were performed with a field-of-view of about $140^{\prime \prime} \times 170^{\prime \prime}$. These required 400 raster steps of $0.35^{\prime \prime}$ each in the East-West (solar-X) direction.

b The values for $\mathrm{X}$ and $\mathrm{Y}$ give the distance of the center of the field-of-view from disk center in the solar-X and $\mathrm{Y}$ directions.

Table 7.2: The relation between magnetic field and the flux of Ca II. In this table is presented the average (av) power-lawindex, the power-law-index that is calculated considering only network structures (nw), emerge network structures (enw) and plage (pl).

\begin{tabular}{|c|c|c|c|c|c|}
\hline Autor & Spatial resolution ["pixel $\left.{ }^{-1}\right]$ & $|\mathrm{B}|[\mathrm{G}]$ & Basal flux ${ }^{\text {回 }}$ & Fitting & Power-law-index ${ }^{\mathrm{b}}$ \\
\hline Loukitcheva et al. (2009) & $4 \& 12$ & $4-200$ & YES & linear, log-log & av: $0.2 ;$ nw: $0.43-0.6$ \\
\hline Rezaei et al. (2007) & 1 & $0-300$ & YES & linear & nw: $0.3-0.51$ \\
\hline Skumanich et al. (1975) & 2.4 & $0-200$ & NO & linear & nw: 1 \\
\hline Schrijver et al. $(1989)$ & $2.4-14.4$ & $0-800$ & YES & linear, log-log & av: 0.6 \\
\hline Nindos and Zirin (1998) & 2 & $0-50$ & NO & linear & nw: 1 \\
\hline Harvey and White (1999), & 2 & $17-785$ & $\mathrm{NO}$ & $\log -\log$ & pl: 0.48 ; nw: 0.56 , enw: 0.7 \\
\hline
\end{tabular}

a The calculation and subtraction of the basal flux

$\mathrm{b}$ Loukitcheva et al. (2009) and Rezaei et al. (2007) showed that magnetic flux density and intensity are independent in the internetwork area. 
7 Emission of solar chromospheric and transition region features related to the underlying magnetic field

Table 7.3: Properties of magnetic field and spectral line observed

\begin{tabular}{|c|c|c|c|c|}
\hline instrument & $\lambda[\AA]^{\circledR]}$ & line / feature & $\log T[\mathrm{~K}]^{\mathrm{d}]}$ & 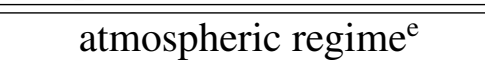 \\
\hline \multirow{2}{*}{ SDO $\begin{array}{l}\text { HMI } \\
\text { AIA }\end{array}$} & 6173 & magnetogram ${ }^{b}$ & - & photosphere \\
\hline & 1600 & continuum ${ }^{\mathrm{C}}$ & $<3.6$ & upper photosphere / T-min ${ }^{[]}$ \\
\hline \multirow{5}{*}{ IRIS } & 2796.9 & Mg II k1r & 3.6 & $T$-min / lower chromosphere \\
\hline & 2796.6 & $\mathrm{Mg}$ II k2r & 3.8 & middle chromosphere \\
\hline & 2796.4 & Mg II k3 & 3.9 & upper chromosphere \\
\hline & 1335.7 & $\mathrm{C}_{\text {II }}$ & 4.6 & low transition region \\
\hline & 1393.8 & Si IV & 4.9 & transition region \\
\hline
\end{tabular}

a The AIA $1600 \AA$ images are acquired in a roughly $50 \AA$ wide wavelength band. For lines observed with IRIS the wavelength of the respective features of the $\mathrm{Mg}$ II $\mathrm{k}$ line or the rest wavelength of C II and Si IV are given (see Sect.7.2.2.1).

b In active regions HMI can also provide the full vector of the magnetic field. Here we use only the HMI data for the line of sight component.

c This $1600 \AA$ A band contains also the C IV doublet at $1548 \AA$ and $1550 \AA$. Except for small patches of strongly enhanced emission around $10^{5} \mathrm{~K}$ this band does not show transition region structures on the disk (e.g., compare panels $b$ and $d$ in Fig.7.2.

d The formation temperatures for the $1600 \AA$ continuum and the $\mathrm{Mg}$ II k line features are taken from Vernazza et al. (1981), the values for C II and Si IV are taken from Peter et al. (2006).

e This ordering should be a guideline only, in particular when thinking of the dynamics in the solar upper atmosphere.

f $T$-min denotes the temperature minimum.

\subsubsection{Data reduction}

\subsubsection{IRIS spectral maps}

After standard procedures to correct the IRIS level-2 data for dark current, flat field and geometric distortions, we apply a five-step preparation procedure. This is shown schematically in Fig.7.1.

First we correct the IRIS raster data for solar rotation. The next and main step is the production of monochromatic maps to obtain spectrally pure images of the line profile features. In the case of $\mathrm{Mg}$ II we locate the position of the self-reversal, $\mathrm{k} 3$, and the maximum of the red wing, $\mathrm{k} 2 \mathrm{r}$, and the emission at the $\mathrm{k} 3$ minimum and the $\mathrm{k} 2 \mathrm{r}$ peak. For this we employ the IRIS reduction software available in SolarSof 1 . For the k1r, the minimum to the red wing of the $\mathrm{Mg}$ II, we use a $0.7 \AA$ wide window around the expected wavelength of k1r (cf. Table 7.3), apply a spline interpolation, and calculate the minimum intensity. The optically thin line of Si IV shows a single-peaked spectrum almost everywhere (for exceptions see e.g. Peter et al.2014), and we apply a spline interpolation to calculate the peak intensity. The line of $\mathrm{C}_{\text {II }}$ is not optically thin and shows signatures of a self-reversal, in particular in plage-like regions. Therefore we do not use the peak intensity but calculate the total line intensity (integrated over the line after subtraction of the continuum). Still, if one would use the peak intensity for $\mathrm{C}$ II the results remain basically unchanged (because even at the high spectral resolution of IRIS the line appears

\footnotetext{
${ }^{1}$ iris_get_mg_features_lev2.pro available at SolarSoft, http: //www. lmsal.com/solarsoft/.
} 


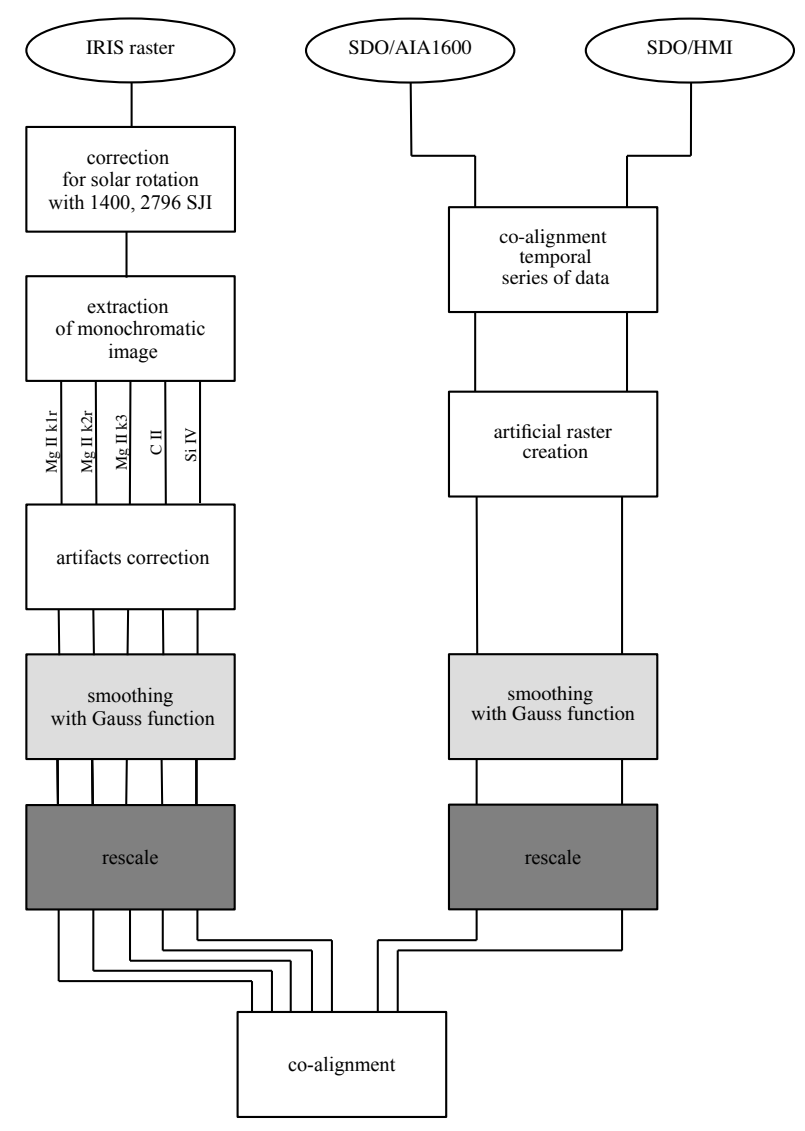

Figure 7.1: Flowchart of data preparation process. See Sect.7.2.2.

single peaked in a large part of the field-of-view, in particular in more quiet regions at low signal-to-noise).

After the extraction of the emission of the line-profile features we corrected for artefacts. Mainly these are rows or columns of bad data (missing data or obvious problematic count rates). These data points were replaced with the interpolated data from adjacent pixels. The IRIS spectral maps are easily aligned spatially among themselves through the fiducial mark on the slit.

We want to investigate the data at different spatial resolutions to study the effect of the resolution on the relation of the upper atmosphere emission to the magnetic field. For this we convolve the data with a Gaussian to reduce the spatial resolution and finally bin the smoothed data to the required resolution. Here we use plate scales of 0.5, 1.5, 3.0 and $6.0^{\prime \prime} /$ pixel.

From this procedure we obtain five rotation corrected, monochromatic, artefact free and rigidly aligned IRIS images for each of the spatial scales for each or the regions listed in Table7.1, 


\subsubsection{SDO imaging and surface magnetic field}

For the SDO data we employ a four-step procedure that is also described schematically in Fig.7.1. Below we describe the procedure for the AIA $1600 \AA$ Aata, but the HMI data are prepared in the same way.

First we extract the time series of the AIA data for the whole time of the raster scan and align this time series. The main step is then to extract the AIA data at the position of the IRIS slit in the AIA image closest in time. Through this we create a raster map of AIA data (reconstructed raster map) that is co-temporal to the IRIS raster maps. This step is crucial to account for the changes in the AIA $1600 \AA$ channel (and the HMI data) during the comparably long time the raster maps are acquired (cf. Table 7.1). This ensures to have SDO data that are co-spatial and co-temporal with the IRIS maps - prerequisite to get a reliable relation between the data products of the different instruments. Just like for the IRIS data we create SDO data sets at four spatial scales $\left(0.5,1.5,3.0\right.$ and $6.0^{\prime \prime} /$ pixel $)$, i.e., first convolving with a Gaussian and then re-binning.

In the final step combining the IRIS and SDO data we align the IRIS maps with the SDO data. For this we first align the Mg II k1r maps with the AIA $1600 \AA$ artificial raster maps, and these to the HMI magnetograms (more precisely to the artificial raster map of the HMI magnetograms).

In summary, after this procedure we have seven maps (as listed in Table 7.3) that allow us to relate the emission from the photosphere through the chromosphere and transition region to the surface magnetic field. These are available at different spatial scales from 0.5 to $6^{\prime \prime} /$ pixel to test the role of spatial resolution, and for three different solar features, namely active region, quiet Sun, and coronal hole (cf. Table 7.1). In Fig.7.2 we show some of these maps for the active region.

\subsubsection{Regions of interest}

To study the relation of the emission from the upper atmosphere to the magnetic field and the flux-flux relations we investigate different types of regions. For these we will derive the correlations and power-law indices at different resolutions (see Sect.7.3) and compare the properties of these different regions of interest.

(1) Active region (without sunspots or pores): This is almost the full field-of-view of the active region data set (cf. Fig.7.2). This region consist also relatively quiet areas and extended (enhanced) network areas, decaying active region (upper part of image) and a region of emerging flux (between the upper two yellow rectangles, associated with some of the strongest brightenings in Mg II k1r). However, sunspots and pores would significantly alter the general relations. For example in sunspots the $\mathrm{Mg}$ II lines differ significantly from the rest of the solar disc in that they do not show the self-reversal feature ( $\mathrm{h} 3$ and $\mathrm{k} 3$ ), but they are singly peaked. Therefore we define the active region as the area that encompasses the full raster map (red rectangle in Fig.7.2) except the regions covered by sunspots (yellow rectangles in Fig.7.2). This is similar to the definition in Schrijver et al. (1989). 

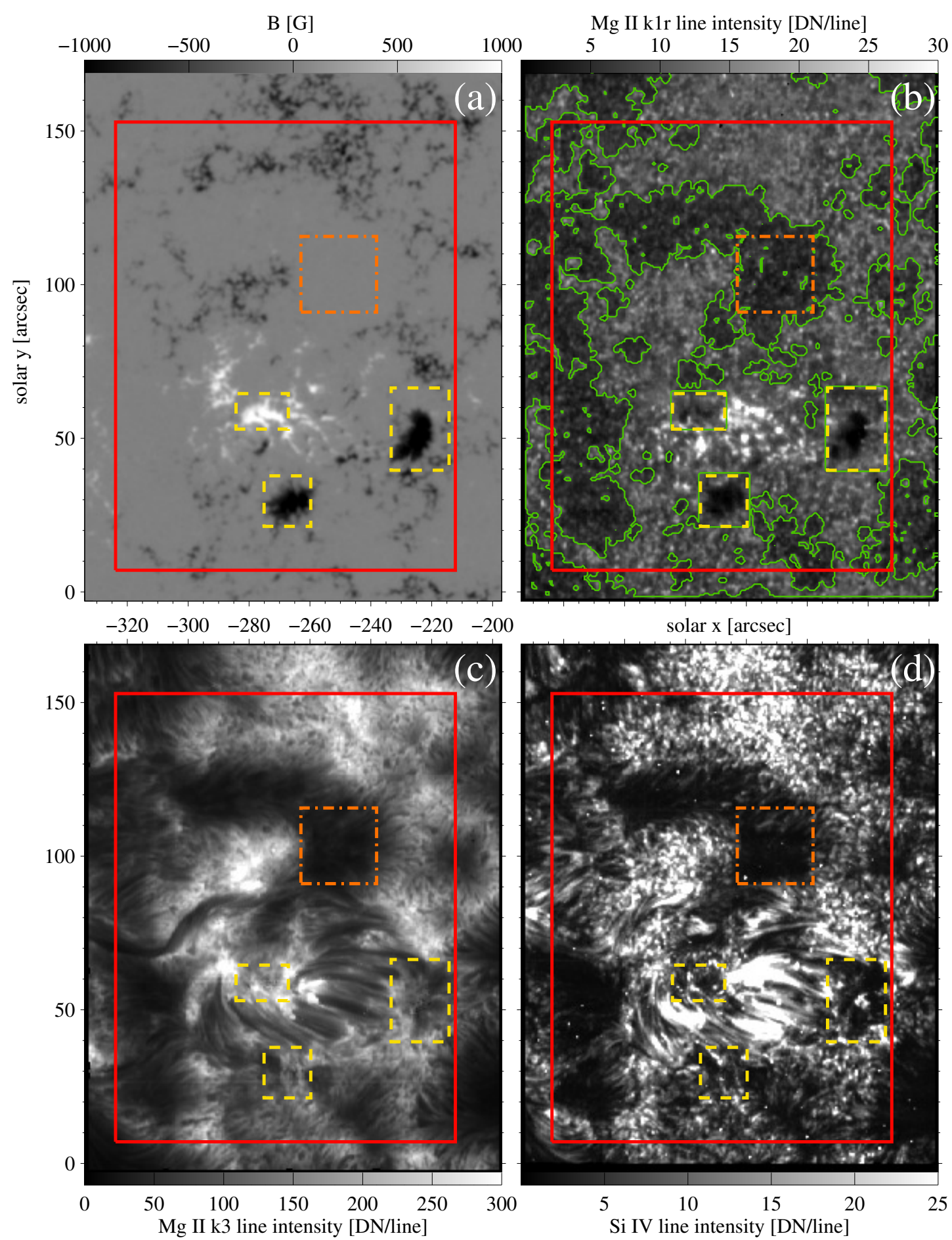

Figure 7.2: Active region from IRIS raster scan and magnetic field context from HMI. The images display the active region 11850 (cf. Table 7.1). The panels show (a) the SDO/HMI line-of-sight magnetogram and the raster maps by IRIS in (b) Mg II k1r, (c) Mg II k3, and (d) Si Iv. The lines and contours highlight the regions used to define the active region and plage areas (see Sect.7.2.3). The three yellow rectangles indicate the location of pores and sunspots, the large red rectangle the full extent of the active region. The green contours (only in panel b) show the location of the plage area. The threshold for the definition of the plage area was calculated in the orange rectangle indicating a quiet region. 
(2) Plage: A large part of the full active region scan is covered by emission with very low intensity from the chromosphere and the transition region. Therefore, we exclude these data points for the definition of the plage regions. For this we estimate the average and standard deviation, $\sigma$, in the images of the AIA $1600 \AA$ channel in a larger patch of a quiet region (orange rectangle in Fig.7.2 b). We then define plage as the region where the AIA $1600 \AA$ emission is more than $2 \sigma$ above the average; still excluding the sunspots. According to this definition, (enhanced) network areas are counted to plage. This plage is shown by the green contours in Fig. 7.2.

(3) Quiet Sun (QS): For comparison we also check the relations within quiet Sun regions. Unfortunately we cannot use quiet regions from the active region data set shown in Fig.7.2 for this analysis. This is because the exposure time is too short to give sufficient signal in particular in $\mathrm{C}$ II and $\mathrm{Si}$ IV, and the quiet regions cover only a small portion of the field-of-view. Therefore we located another large dense raster of IRIS that has sufficient signal-to-noise ratio (cf. Table 7.1). Here the field of view is fully covered by quiet Sun with no active regions nearby.

(4) Coronal hole $(\mathbf{C H})$ : Just as for the quiet Sun we also compare the relations in a coronal hole region. Here again we have to investigate another data set and chose one where the IRIS raster was fully within a (on-disk) coronal hole (cf. Table 7.1).

When investigating the relation of the magnetic field to the upper atmosphere emission, the mag-flux relations, we consider only those locations with (an absolute value of) the magnetic field strength of up to $200 \mathrm{G}$. This is the same threshold as used by Loukitcheva et al. (2009). Other studies used higher thresholds, e.g. Schrijver et al. (1989) used field strengths up to $800 \mathrm{G}$. However, all the four regions of interest considered here do not contain sunspots or pores, and those were also excluded in the study of Schrijver et al. (1989). Therefore there are only few data points left where the magnetic field strength as recorded by the moderately resolving HMI instrument is above $200 \mathrm{G}$. (Of course, with a high-resolution instrument one can detect and resolve kilo-Gauss flux tubes even in the internetwork quiet Sun; e.g. Lagg et al. 2010). To avoid the poor statistics for high magnetic field strengths and because the vast majority of the data points are to be found below $200 \mathrm{G}$, we restrict the analysis to flux densities below this value.

\subsection{Methods}

Based on the aligned images of the magnetograms, or rather of the absolute value of the pixel-averaged magnetic field strength $|B|$, and the intensities $I$ of the emission line features we study the relation between the emission from different parts of the solar atmosphere and the underlying magnetic field, here called mag-flux relations (Sect.7.3.2) For this we calculate a correlation coefficient and fit a power-law function to characterise the respective relations. To get reliable mag-flux relations we first have to subtract the basal flux, i.e. the emission that originates from the atmosphere in the absence of magnetic field (Sect.7.3.1). Finally we also study flux-flux relations, i.e. the relation of the emission from different parts of the atmosphere (Sect.7.3.3). 
Later in Sects.7.4 and 7.5 we will discuss different solar features over a range of spatial resolutions. In the present section we describe the method using the example of the active region data set (Sect.7.2.3.1) at a spatial scale of 1.5"/pixel.

\subsubsection{Basal flux}

When determining the relation between the magnetic field strength and the intensity of a chromospheric or coronal emission line, e.g. through a power law, the basal flux plays a critical role. This basal flux is the emission that is present even in the absence of magnetic field. Usually this is interpreted as representing emission from an atmosphere heated purely by acoustic waves and shocks (Schrijver et al.|1989, Rutten and Uitenbroek|1991). In the scatter plots of radiative flux vs. magnetic flux density for a number of emission features in Fig.7.3 it is clearly evident that there is a minimum radiative flux at low magnetic flux density. Under the presence of magnetic field also the emission powered by magnetic heating of the atmosphere is present, and thus the radiative flux will increase with the magnetic field strength.

The concept of the basal flux was introduced by Schrijver et al.(1989). In their original work they defined the basal flux simply as the minimum intensity in the field-of-view investigated on the Sun. Implicitly they assumed that one would find the lowest intensity value only at the lowest magnetic field strengths which will be zero field strength somewhere in the region considered. Thus this would represent the basal flux. However, it is clear that this method is sensitive to outliers of the intensity maps. One can minimise this effect by investigating a time series of images (Schrijver 1992). However, this is not feasible for our data, which are maps produced by scanning with a spectrograph slit acquired over a comparably long time. Therefore, we modify the original procedure of Schrijver et al. (1989) to account for outliers.

Our method to determine the basal flux is based on dividing the collection of pixels with low magnetic field strength into bins according to magnetic field strength. In each bin we then determine the minimum value of the intensity and define the median of these minimum values in the bins as the basal flux. In the absence of outliers this produces the same results as Schrijver et al. (1989), but it can also cope with outliers. Of course, we repeat this procedure for each of the emission features (from AIA and IRIS; cf. Table 7.3) to derive the basal flux separately for each line or spectral feature.

Details of our method are given in Appendix 7.7.1. When calculating the basal flux we investigate only pixels in the images with magnetic field strengths below $4 \mathrm{G}$, which is roughly half of the noise level of the HMI data we use for the magnetic field. This ensures that we only look at the regions that appear to be field-free (to HMI). It turns out that the best magnetic bin size for our analysis is $1 \mathrm{G}$ (see Appendix 7.7.1), i.e. we subdivide the collection of low magnetic field pixels into four bins. The median of the minimum intensities in the bins is then used as the basal flux, $I_{0}$, when performing the power-law-fits in Sect.7.3.2.2. 
7 Emission of solar chromospheric and transition region features related to the underlying magnetic field
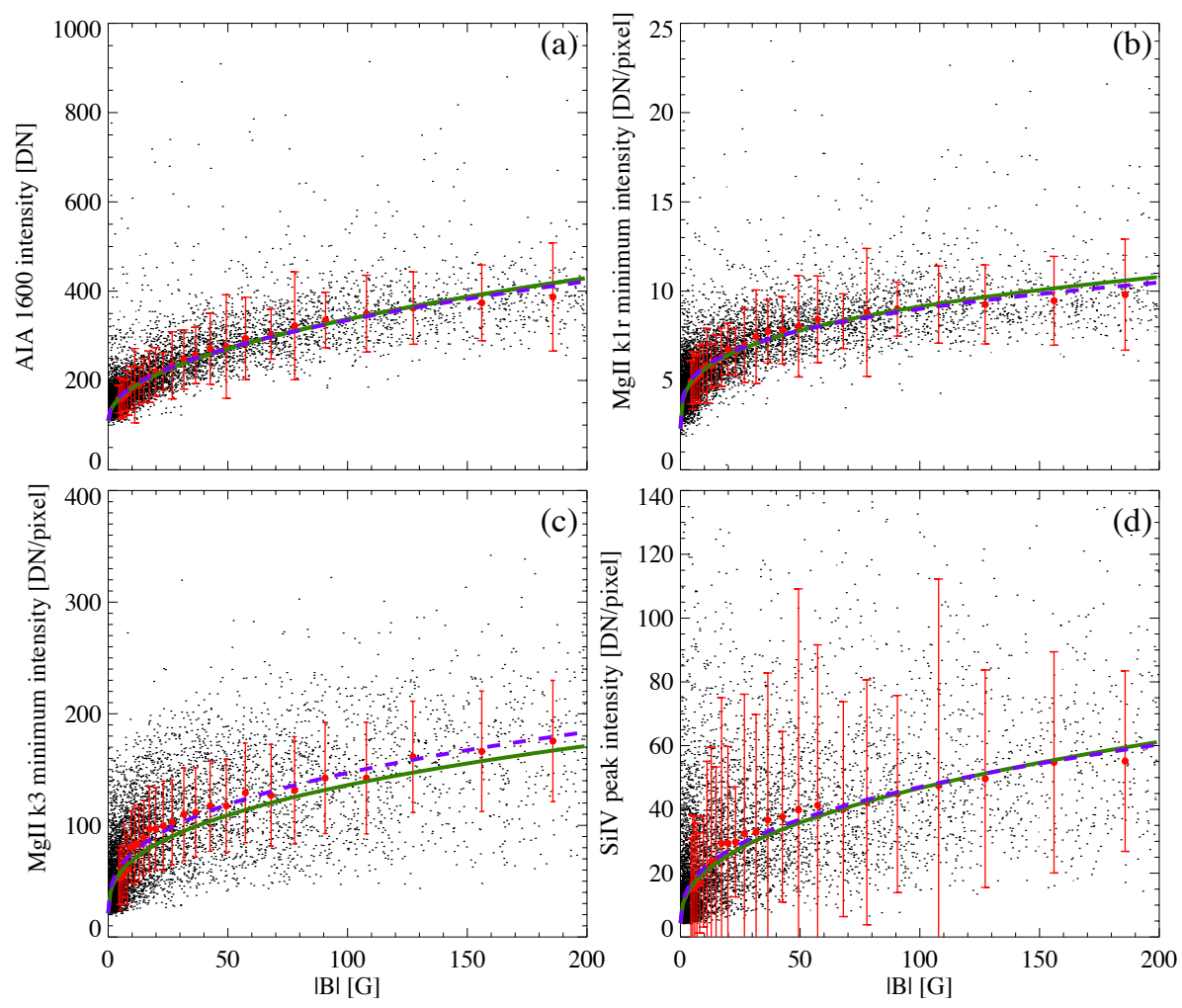

Figure 7.3: Relation of upper atmosphere emission to the magnetic field. While we prepared these plots to calculate correlations and power-law-fits for all emission features listed in Table 7.3, for brevity we plot here only the relations involving (a) AIA $1600 \AA$, (b) $\mathrm{Mg}$ II k1r, (c) Mg II k3, and (d) Si Iv. The power-law functions fitted to all data points are represented by (green) solid curves. The red bars indicate the the average and standard deviation within bins in magnetic field strength $|B|$, where we grouped the bins for equal numbers of data points (here 200 points). The blue dashed curves show power-law-fits to the binned data. The data shown in these examples are for the active region set as defined in Sect.7.2.3 (red rectangles without yellow rectangles in Fig.7.2). The same data are plotted in Fig.7.10 on a double-logarithmic scale. See Sects.7.3.2.1 and 7.3.2.2.

\subsubsection{Relating the upper atmosphere emission to the surface mag- netic field: mag-flux relations}

\subsubsection{Mag-flux: correlation}

With the basal flux subtracted we can now statistically relate the upper atmosphere emission to the magnetic field though the mag-flux relations. In the resulting scatter plots of emission vs. magnetic field we find clear relations of the respective two quantities, in particular when comparing to the different features of the Mg II line and AIA $1600 \AA$ (see Fig. 7.3).

Because these relations appear to be nonlinear we use the Spearman's rank order correlation coefficient (in short: Spearman correlation) for a quantitative analysis of this relation. The Spearman correlation measures any monotonic relation between two vari- 
ables, regardless of the functional form, and is not sensitive to outliers. This is in contrast to the cross-correlation coefficient that is frequently used, but strictly speaking works only to characterise a linear relation. In general, for a non-linear relation the cross-correlation will underestimate the correlation between the two quantities (for more details and examples see Sect.7.3.3.1). Depending on the spatial resolution of the data the Spearman correlation coefficients are in the range from 0.6 to 0.9 . This shows that there is a clear relation that motivates the application of a power-law fit.

\subsubsection{Mag-flux: power-law relation}

The scatter plots (Fig.7.3) are clearly non-linear, and plotting them on a double-logarithmic scale suggests a power-law trend between the magnetic field and intensities (cf. Fig.7.10). The same was noticed before for spatially resolved observations between e.g. between $\mathrm{Ca}$ II and the magnetic field (Schrijver et al. 1989) or $1600 \AA$ images and magnetic field (Loukitcheva et al.2009). Therefore we assume a power-law here, too, i.e.

$$
I^{\prime}=I-I_{0}=a \cdot|B|^{b}
$$

where $I$ is the intensity of the emission feature, $I_{0}$ the basal flux, $|B|$ the absolute value of the line-of-sight photospheric magnetic flux density, and $a$ a scaling parameter. Most importantly, $b$ is the power-law-index.

Here we employ two methods to conduct the power-law-fit to determine the powerlaw-index, $b$. Three more methods are illustrated in Appendix 7.7.2, which all give similar results underlining the robustness of our findings.

In the first method we directly apply a least-squares fit to the power-law-function in Eq.7.1 to each of the scatter plots showing $I-I_{0}$ vs. $|B|$, where we apply statistical errors (Poisson weights) for the uncertainties in $I-I_{0}$. The power law fits obtained this way are shown in Fig.7.3 (green solid lines).

We also applied an alternative second method for the power law fitting in which we bin the data points in magnetic field strength. For a consistent signal-to-noise we used bins with an equal number of data points (here 200) starting from $4 \mathrm{G}$, i.e. from above the level for the basal flux calculation (Sect.7.3.1), to $200 \mathrm{G}$. These bins are plotted in Fig.7.3 as bars, where the height of the bars indicates the standard deviation of the intensity values in the respective bin. For the power-law-fit we fit the average intensities in the bins using the standard deviations in the bins as weights. The resulting fits are shown in Fig.7.3 (blue, dashed line) and they are virtually identical with the method of fitting all points directly (with the exception of $\mathrm{Mg}$ II k3, where the slopes are within a few percent).

\subsubsection{Flux-flux relations}

The relations of the radiative fluxes from different parts of the atmosphere, the flux-fluxrelations, contain valuable information on how the structure and possibly the governing processes change throughout the upper atmosphere. For many stellar observations there is no information available on the magnetic field (distribution) on the stellar surface. Even in those cases flux-flux relations are available as long as those stars are observed in different wavelengths originating from different parts of the upper atmosphere. 
7 Emission of solar chromospheric and transition region features related to the underlying magnetic field

Table 7.4: Mutual relation of emission features for the active region set. The Spearman's Rank order correlation coefficients are presented in the blue part, the cross-correlation in the red part.

\begin{tabular}{|c|c|c|c|c|c|c|}
\hline \multirow{2}{*}{\multicolumn{2}{|c|}{ correlation }} & \multicolumn{3}{|c|}{ Mg II } & \multirow{2}{*}{$\mathrm{C}_{\mathrm{II}}$} & \multirow[b]{2}{*}{ Si IV } \\
\hline & & $\mathrm{k} 1 \mathrm{r}$ & $\mathrm{k} 2 \mathrm{r}$ & k3 & & \\
\hline \multirow{3}{*}{ Mg II } & k1r & 1 & 0.63 & 0.54 & 0.55 & 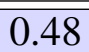 \\
\hline & $\mathrm{k} 2 \mathrm{r}$ & 0.53 & 1 & 0.88 & 0.84 & 0.70 \\
\hline & k3 & 0.42 & 0.88 & 1 & 0.81 & 0.62 \\
\hline \multicolumn{2}{|c|}{$\mathrm{C}_{\text {II }}$} & 0.24 & 0.26 & 0.22 & 1 & 0.80 \\
\hline \multicolumn{2}{|c|}{ Si IV } & 0.22 & 0.23 & 0.17 & 0.16 & 1 \\
\hline \multicolumn{2}{|c|}{$\begin{array}{l}\text { power-law index } \\
\text { to } \mathrm{Mg} \text { II k1r }\end{array}$} & - & $\begin{array}{c}1.4 \\
\pm 0.2\end{array}$ & $\begin{array}{c}1.9 \\
\pm 0.01\end{array}$ & $\begin{array}{c}1.9 \\
\pm 0.2\end{array}$ & $\begin{array}{c}2.5 \\
\pm 0.4\end{array}$ \\
\hline
\end{tabular}

The correlation coefficients are based on the raster images at the original IRIS spatial resolution without the subtraction of the basal flux. See Sect.7.3.3.1 for the correlation coefficients and Sect.7.3.3.2 for the power-law indices.

In principle, the relation between the magnetic field and the emission from the upper atmosphere, the mag-flux-relations, are directly linked to the processes governing the physics of the upper atmosphere. However, when observing at high resolution, on the Sun the mag-flux relations might be obscured by the structure of the magnetic field. The emission from the chromosphere and transition region originates from heights in the solar atmosphere of at least $1 \mathrm{Mm}$ and above. For example the transition region is located over a height range from about $2 \mathrm{Mm}$ to $5 \mathrm{Mm}$, intermittent both in time and space (e.g. Peter 2013). Therefore even slightly inclined field originating from small magnetic flux tubes in the photosphere will prevent seeing a clear relation between magnetic field and emission when observing at a spatial resolution corresponding to $1 \mathrm{Mm}$ or better. Therefore we also investigate the flux-flux relation in the spatially resolved data. In the following we will concentrate on the IRIS data alone, i.e. on the emission from the features of $\mathrm{Mg}$ II, $\mathrm{C}$ II and Si IV. This has the advantage that these lines are observed through the same slit and the spatial alignment of the data is easily ensured through the fiducial marks on the slit (De Pontieu et al. 2014).

\subsubsection{Flux-flux: correlation}

Just as for the mag-flux relations, to quantify the relation between the different radiative fluxes from the upper atmosphere we use the Spearman correlation, because it is more appropriate for non-linear relations. The respective Spearman correlation coefficients for the active region set are listed in the upper right half of the correlation matrix in Table 7.4 (shaded blue). As for the correlation to the magnetic field, here the correlation also drops with temperature. For example in the top row of Table 7.4, which shows the correlation to $\mathrm{Mg}$ II $\mathrm{k} 1 \mathrm{r}$, the correlation for $\mathrm{Mg}$ II $\mathrm{k} 2 \mathrm{r}$ is higher than the one for $\mathrm{Si}$ IV.

To illustrate the difference between the Spearman correlation and the cross-correlation, in Table 7.4 we also give the respective cross-correlation coefficients (lower left part of the matrix, shaded red). As mentioned in Sect.7.3.2.1 in general the cross-correlation coefficients are significantly lower than the Spearman correlation, which is basically because 
the relations between these quantities are non-linear. We note that in their study Schmit et al. (2015) found a correlation between $\mathrm{Mg}$ II h1v to h2v of only 0.33 for the quiet Sun using a cross correlation technique which is lower than the 0.38 we find using the Spearman correlation for the quiet Sun set. This underlines the importance of using the Spearman correlation in the presence non-linear relations.

\subsubsection{Flux-flux: power-law relation}

To derive the power-law-index for the flux-flux relations we go a different way than for the mag-flux relations, where we fit directly power-law functions. In a scatter plot (or rather a 2D histogram or probability density function, PDF) of the flux-flux relations in a doublelogarithmic diagram, the cores of distributions are quite symmetric and seem to have an ellipsoidal shape (see Fig.7.4). For this the basal flux is subtracted first, of course. We fit an ellipse to the PDFs (on log-log scale), with the major axis then representing the slope, i.e. the power-law index. Comparing to a conventional power-law fit we found that the results are consistent, but the the ellipse fitting turned out to be more robust. Thus when deriving the flux-flux relations of the different regions for the different spatial resolutions we used the power-law indices derived from the ellipse fitting.

To obtain a power-law-index we use a two-step procedure. First we fit ellipses to 50 contour levels of the PDF ranging from $25 \%$ to $75 \%$ of the peak value of the PDF. We then fit a Gaussian along the average major axis of the fitted ellipses to determine the full width at half maximum (FWHM) of the PDF. Finally we fit three ellipses to the PDF at the contour levels with $[1,1.5,2] \times$ FWHM. The average of the major axis for these three ellipses defines the slope of the power-law function, the difference between the minimum and maximum values we use as an estimate for the error of the slope.

The results for this procedure in the case of the active region set are illustrated in Fig.7.4. Here we plot the fitted ellipses (to $1.5 \times$ FWHM) for each of the flux-flux relations along with the power-law (see also Table 7.4). This same procedure is applied to all the different regions-of-interests for the different spatial resolutions, just as for the mag-flux relations.

\subsection{Results}

Here we first discuss the results for the active region set before we compare these to the plage, quiet Sun and coronal hole sets. For the definition of these regions of interest see Sect.7.2.3.

\subsubsection{Active region (without sunspots and pores)}

\subsubsection{The mag-flux relations}

The most previous studies of the (spatial) relation between magnetic field and intensity were based on lower spatial resolution data than used in this paper. To compare our results with previous results and to study the impact of spatial resolution on correlation coefficients and power-law-indices we investigate the data sets at spatial samplings from 

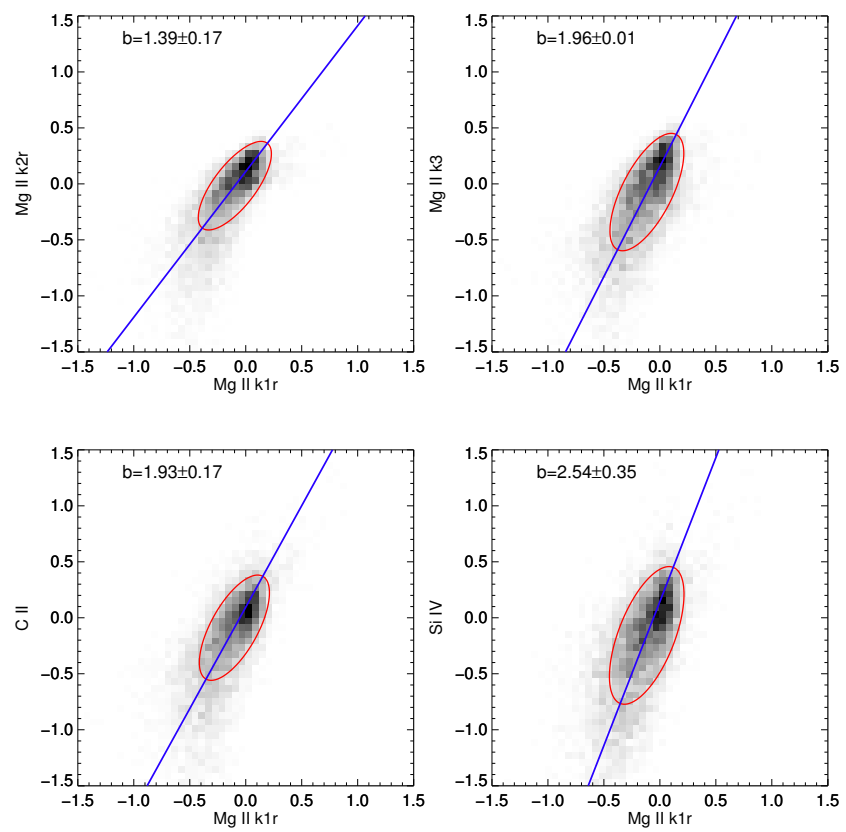

Figure 7.4: Probability density functions (PDFs) for the flux-flux relation for the active region set for a spatial resolution of $1.5^{\prime \prime}$. Here the respective basal fluxes are subtracted. The intensities are normalised to the respective median value and are plotted on a logarithmic scale. The red ellipses show the fit to the contour level at $1.5 \times$ FWHM. The blue lines indicate the power-law-slopes calculated as the average from the ellipse fits of the contour levels at $[1,1.5,2] \times$ FWHM. See Sect.7.3.3.2

$0.5^{\prime \prime}$ to $6.0^{\prime \prime}$ per pixel. For each resolution the basal flux was calculated separately and then subtracted from the intensities to derive the correlation and power-law indices.

The correlation between the emission and the underlying magnetic field (cf. Sect.7.3.2.1) drops monotonically with temperature (in an average sense; see Table 7.3 for the temperatures). This trend is illustrated in Fig.7.5 a that shows the Spearman correlation between emission and magnetic field for four spatial resolutions. This trend is seamlessly connecting also across instruments from the AIA $1600 \AA$ A channel to Mg II k1r observed with IRIS. When checking different spatial resolutions, we find the trend of the correlation from the temperature minimum to the transition region to be independent of resolution. However, for the same emission feature we see a higher correlation at lower spatial resolution. This latter result is consistent with the Schrijver et al. (1989) analysis that concentrated on the $\mathrm{Ca}$ II line. We will discuss a physical scenario for this drop of the correlation coefficient in Sect.7.5.1,

The power-law index characterising the relation between the emission features and the magnetic field (cf. Sect.7.3.2.2) shows a more peculiar behaviour. The index grows monotonically from $\mathrm{Mg}$ II to the transition region and will be discussed in Sect.7.5.3. However, the power-law index for the AIA $1600 \AA$ channel is higher than for the other emission features, giving the plot in Fig. $7.5 \mathrm{~b}$ the appearance of a hockey stick. In Sect.7.5.2 we will show that the drop from AIA $1600 \AA$ to $\mathrm{Mg}$ II k1r is real and actually can be smoothly followed through the temperature minimum region. This is why we 

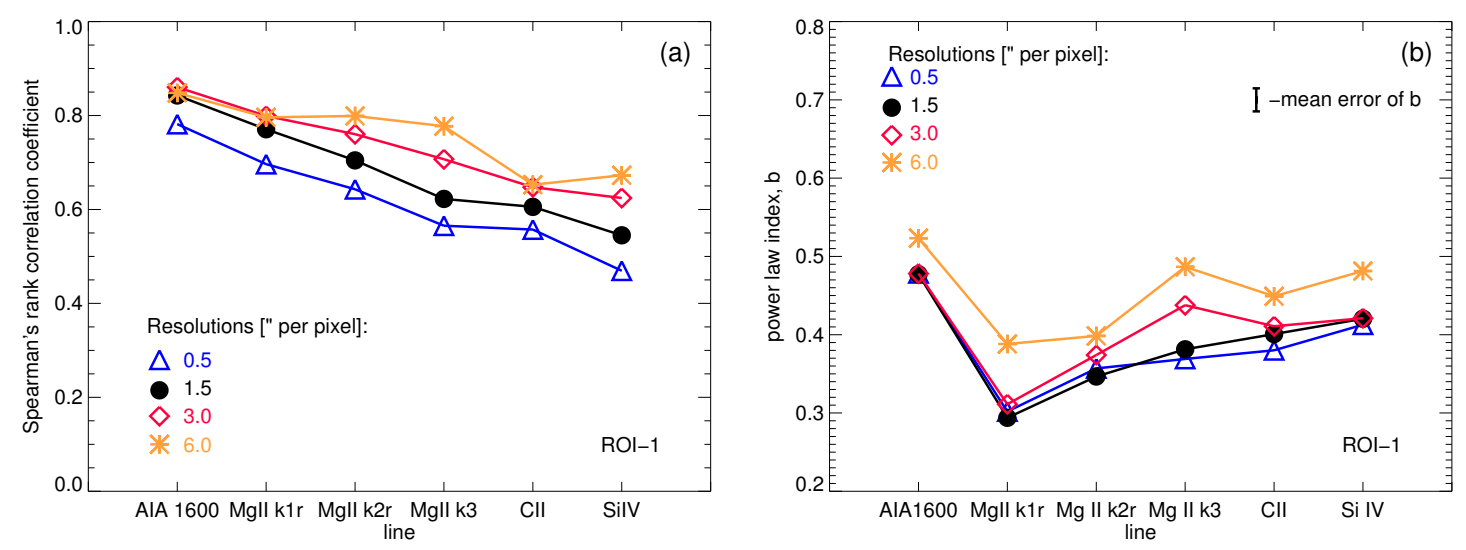

Figure 7.5: Relation of the upper atmosphere to the underlying magnetic field for the active region set. Panel (a) shows the Spearman correlation of the various emission features to the photospheric magnetic field, panel (b) the power-law index from the power-law fit of the relation of emission to magnetic field. The emission features are ordered according to the approximate formation temperature (cf. Table 7.3). The different colours show the results based on data at different spatial resolutions, here represented by plate scales from $0.5^{\prime \prime}$ to $6^{\prime \prime}$ per pixel. See Sect.7.4.1.1.

show connecting lines between the data points for AIA $1600 \AA$ and Mg II k1r in Fig. $7.5 \mathrm{~b}$.

This same hockey-stick trend of the power-law index is present irrespective of spatial resolution. For the resolutions from $0.5^{\prime \prime}$ to $3^{\prime \prime}$ per pixel we find almost the same powerlaw indices. This is in agreement with Schrijver et al. (1989) who found the power-law index to be independent of resolution (in the case of $\mathrm{Ca} \mathrm{II} \mathrm{H}$ and $\mathrm{K}$ ). However, for coarse resolutions we find a slight increase of the power-law index for all the emission features that seems to be larger than the error bars (Fig.7.5p).

While the correlation and (to a lesser degree) the power-law index depend on the spatial resolution, the overall trend remains unaffected by the resolution. Therefore the variation of these parameters throughout the atmosphere from the temperature minimum through the chromosphere into the transition region is a robust result. This includes the monotonic drop of the correlation coefficient as well as the hockey-stick-type trend of the power-law index.

\subsubsection{The flux-flux relations}

In order to be independent of the impact that combining different instruments and possible misalignments might have, we also study the flux-flux relations of the emission features seen by the IRIS spectrograph alone (cf. Sect.7.3.3.2). When looking at the power-law indices of the emission lines (or their features) with respect to $\mathrm{Mg}$ II $\mathrm{k} 1 \mathrm{r}$ (bottom row of Table 7.4) we find an increase with temperature. This confirms the increase of the powerlaw index for the mag-flux relations for spectral lines forming at temperatures higher than the equivalent of $\mathrm{Mg}$ II $\mathrm{k} 1 \mathrm{r}$ (i.e. the long handle of the hockey stick).

When handling the IRIS data alone, the spatial alignment of the emission features can be done with very high precision, which is ideal for a comparison on how the relations 
7 Emission of solar chromospheric and transition region features related to the underlying magnetic field

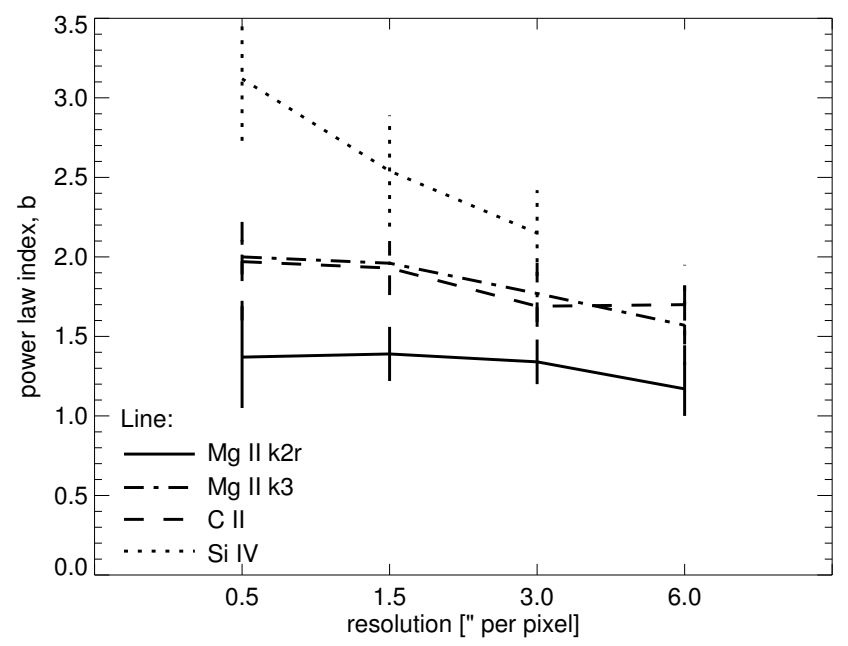

Figure 7.6: Power-law index of the flux-flux relations for different resolutions (here as plate scale). All the power-law indices are derived from the ellipse fits to the doublelogarithmic plots (cf. Fig.7.4) of the respective emission line (feature) versus $\mathrm{Mg}$ II $\mathrm{k} 1 \mathrm{r}$ for the active region set. See Sect.7.4.1.2

change with spatial resolution. When comparing different instruments a (missing) difference between fine and coarse resolution could also originate in imperfections of the alignment — which is eliminated here by using IRIS-only data. In Fig.7.6 we show how the power-law indices of the emission features with respect to $\mathrm{Mg}$ II $\mathrm{k} 1 \mathrm{r}$ change with spatial resolution. For the other $\mathrm{Mg}$ II features as well as for $\mathrm{C}$ II there is no change with spatial resolution. However, for $\mathrm{Si}$ IV a clear trend can be seen with a significantly steeper power-law index at high spatial resolution. (Even though the results for Si IV were not reliable because of the small number of data points when binning). The possible implications of this will be discussed in Sect.7.5.5.

If there would be no scatter in the data, one would expect that from two power law relations between three quantities the third power law relation follows. For example, assume that $\mathrm{Mg}$ II, $\mathrm{C}$ II, and $\mathrm{Si}$ IV denote the emission in the corresponding lines. Then the above statement corresponds to testing the following relation:

$$
\left.\begin{array}{r}
\mathrm{C}_{\text {II }} \propto \mathrm{Mg}_{\text {II }}^{\alpha} \\
\mathrm{Si} \text { IV } \propto \mathrm{Mg}_{\text {II }}{ }^{\beta}
\end{array}\right\} \quad \stackrel{?}{\longrightarrow} \quad\left\{\begin{array}{c}
\mathrm{Si}_{\text {IV }} \propto \mathrm{C}_{\text {II }}^{\gamma} \\
\text { with } \gamma=\beta / \alpha .
\end{array}\right.
$$

For the active region set (1.5" plate scale) from the values of $\alpha$ and $\beta$ in Table 7.4 (and Fig. (7.4) we find $\beta / \alpha=1.3$. This is close to the value of $\gamma=1.21 \pm 0.12$ derived directly from the flux-flux relation between $\mathrm{Si}$ IV and C II (and within the error estimate). Also for other regions of interest and spatial resolutions the values for $\beta / \alpha$ and for $\gamma$ differ by less than the error estimate.

This result implies that the scatter of the emission in the flux-flux relations is independent of the emission itself. Otherwise, when combining two power laws the scatter would alter the power law derived for the third relation. In Sect.7.5.5 we discuss what this implies for the upper atmosphere. 

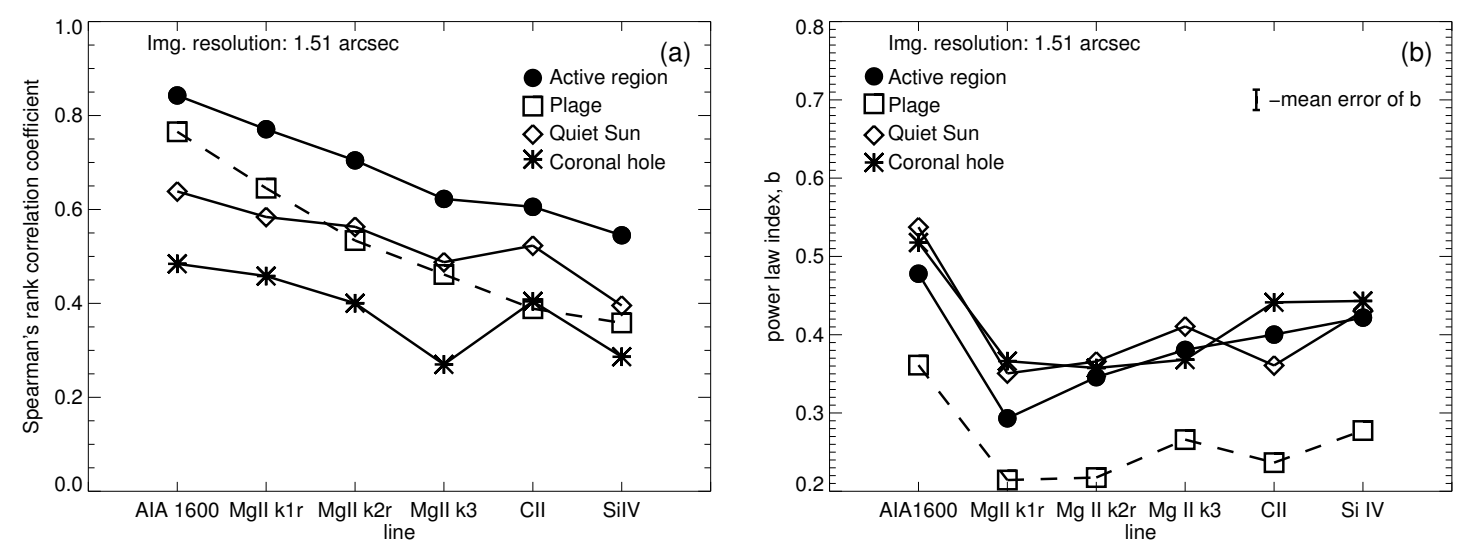

Figure 7.7: Relation of the upper atmosphere to the underlying magnetic field in different parts of the Sun. This figure is similar to Fig. 7.5, but for a spatial scale of 1.5" per pixel for the four regions of interest as defined in Sect.7.2.3. Panel (a) shows the Spearman correlation to the (underlying) magnetic field and panel (b) the power-law index of the power law fit of emission feature vs. magnetic field. The curves for active region (solid with filled dots) are identical to the respective black curves in Fig.7.5. See Sects.7.4.2 to 7.4 .3 .

\subsubsection{The plage area excluding the quiet Sun}

In general the results for the plage area as well as those for the quiet Sun and the coronal hole are similar to the active region. Therefore in this and the following subsection we concentrate mainly on how the results in these other parts of the Sun differ from the active region.

The plage region is defined by excluding the more quiet parts from the active region domain (cf. Sect.7.2.3). Basically this leaves the parts of the active region that are outside sunspots and pores where the chromosphere appears bright.

There are two major differences to the active region: (1) The correlation of the emission features to the magnetic field seems to drop faster with formation temperature. (2) While the overall hockey-stick-type trend of the power-law-index remains unchanged, the power-law indices are significantly lower than in the active region set. These findings are discussed in Sects.7.5.1 and in Sect.7.5.3.

\subsubsection{Quiet Sun and coronal hole}

The results for the quiet Sun and the coronal hole differ mainly in two aspects from the active region data set. The correlations are lower while the power-law indices are still comparable to the active region (without sunspots and plages).

For the correlation between the emission and the magnetic field we see a clear ordering, with the Spearman correlation being lower in quiet sun compared to the active region (and comparable to plage), while the correlation in the coronal hole is even less (Fig.7.7a). However, the trend of the correlation with temperature is similar in all cases in that the correlation drops with formation temperatures. This will be discussed in Sect.7.5.1. 
In contrast to the correlation coefficients, the power-law indices for quiet Sun and coronal holes are comparable to those in the active region, with respect to their values for each emission feature as well as for the trend with formation temperature (Fig. 7.7p). This is surprising, because for the plage regions the lower correlation coefficients go along with reduced power-law indices (cf. Sect.7.4.2), as will be discussed in Sect.7.5.3.

\subsection{Discussion}

The main goal of this study is to shed light on the connection between different temperature regimes in the upper solar atmosphere and the underlying magnetic field. For this purpose, we discuss correlations (Sect.7.5.1) and power-law relations (Sects.7.5.2 to 7.5.4 between magnetic field and intensities as well as the mutual relation between intensities (Sect.7.5.5).

Studies of the relation of the surface magnetic field to the emission have been conducted before, e.g. to Ca II (Schrijver et al. 1989) or the EUV continuum (Loukitcheva et al. 2009). However, these studies did not investigate how the relation of the magnetic field to the emission is changing throughout the chromosphere and into the transition region, an aspect that we concentrate on in this work.

\subsubsection{Correlation between magnetic field and emission}

Our observations show that the correlation with the underlying magnetic field decreases with increasing formation temperatures of the emission feature through the upper atmosphere (Sect.7.4.1.1, Fig.7.5a). The new aspect we highlight here is that a continuous drop of the correlation with the magnetic field is found even within the chromosphere over the formation regions of the $\mathrm{Mg}$ II line features, from $\mathrm{k} 1$ to $\mathrm{k} 2$ to $\mathrm{k} 3$ - in a statistical sense. The high spatial complexity and the intermittent nature of the chromosphere will cause significant deviations from the average correlation, which is clear from the large scatter that is much larger than the measurement errors (seen when plotting, e.g., the emission vs. the magnetic field as in Fig. 7.3).

The simplest explanation of the drop of the correlation is based on the magnetic expansion of the chromosphere (e.g. Solanki and Steiner 1990) that will also be instrumental to interpret the power-law relations later in Sect.7.5.3. Starting from small patches in the photosphere, the hot magnetic flux tubes expand and cover an increasing fraction of the horizontal plane towards higher altitudes, which in a 1D semi-empirical model (e.g. Vernazza et al. 1981) is the same as moves up to higher temperatures. Therefore, the emission in the chromosphere forming at increasingly higher temperatures will fill a larger and larger fraction of the surface area. Naturally, then the correlation between the magnetic field and the emission will decrease with increasing temperature. In addition, the flux tubes can (and many will) be inclined, the inclination often increasing with height (if the field is part of a loop), so that there will be a spatial mismatch between magnetic field and emission. This effect increases with formation height of the emission (in space and temperature if considering a simple stratification like in Vernazza et al. 1981).

For the emission forming at temperatures higher than in the chromosphere, i.e. the transition region, we can expect even less correlation. For lines such as $\mathrm{C}$ II or Si IV at 
least part of the emission will form at the footpoints of loops reaching higher up (see e.g. Peter 2001). As most of these loops will be inclined, this emission will be, in general, mismatched to the underlying magnetic field. Therefore, the decreasing correlation from the low chromosphere into the transition region can be well understood by the geometry of the flux tubes hosting the emitting plasma. Still, it is surprising that despite of all the spatial and temporal variability this average trend of a dropping correlation is so clearly defined.

The drop of the correlation with increasing spatial resolution (Fig. 7.5 a) can be also understood by geometric effects, again in part based on the flux-tube picture. Firstly, when the spatial resolution decreases, the small footpoints in the photosphere get smoothed out, which leads to a better correlation to the upper atmosphere, where the emission forms larger patches because of the fanning out of the flux tubes. Secondly, at lower resolution also flux tubes with an inclination to the line-of-sight will show a match between their footpoints and the emission higher up, leading to higher correlation. And thirdly, when observing at lower resolution, many of the small-scale opposite polarity magnetic concentrations will cancel in the polarisation signal resulting in a smoother magnetic field map, which in turn gives a better correlation to the emission in the fanned-out part of the flux tubes.

In their original work Schrijver et al. (1989), suggested that a typical spatial scale should exist because they found the scatter about the mean relation of (Ca II) emission vs. magnetic field to be independent of resolution. The scatter only dropped once they degraded the spatial resolution to about 14". However, it is not clear how such a typical length scale for the magnetic field patches should be compatible with our finding that there is a continuous drop of the correlation between emission and magnetic field from subarcsec resolution to several arcsec. Schrijver et al. (1989) also suggested that the increase of the correlation when degrading the resolution could be understood by the resolution element becoming comparable in size to the characteristic length of the chromospheric oscillation. However, here we wonder how this effect should explain the decrease of the correlation also for the transition region lines, which do not show the characteristic oscillations seen in the chromosphere.

At first sight it is surprising that in general the correlation for the plage area is slightly lower than for the active region and also drops faster with formation temperature (Fig.7.7.7). However, in our definition the plage region is the part of the active region (without sunspots and pores) that excludes the parts where the chromosphere is dark (see Fig.7.2, Sect.7.2.3). Leaving out the darker part of the chromosphere makes the emission maps more homogeneous and thus the contrast to the highly structured magnetic field becomes less. Consequently the correlation coefficients will be lower.

In the quiet Sun and the coronal hole data sets the correlation is systematically lower than in the active region (Fig.7.7 ), too. This is to be expected, because in the quiet Sun and coronal hole there will be a smaller fraction of magnetic structures reaching up into the chromosphere and transition region. Thus unlike in the active region there is a less clear connection from the photosphere to the upper atmosphere.

Interestingly, the correlation in the coronal hole is significantly lower than for the quiet Sun, even low in the chromosphere. This is a surprise because in general the differences between coronal hole and quiet Sun are considered to be small at chromospheric levels. However, Wiegelmann and Solanki (2004) showed through a potential field extrapola- 
7 Emission of solar chromospheric and transition region features related to the underlying magnetic field
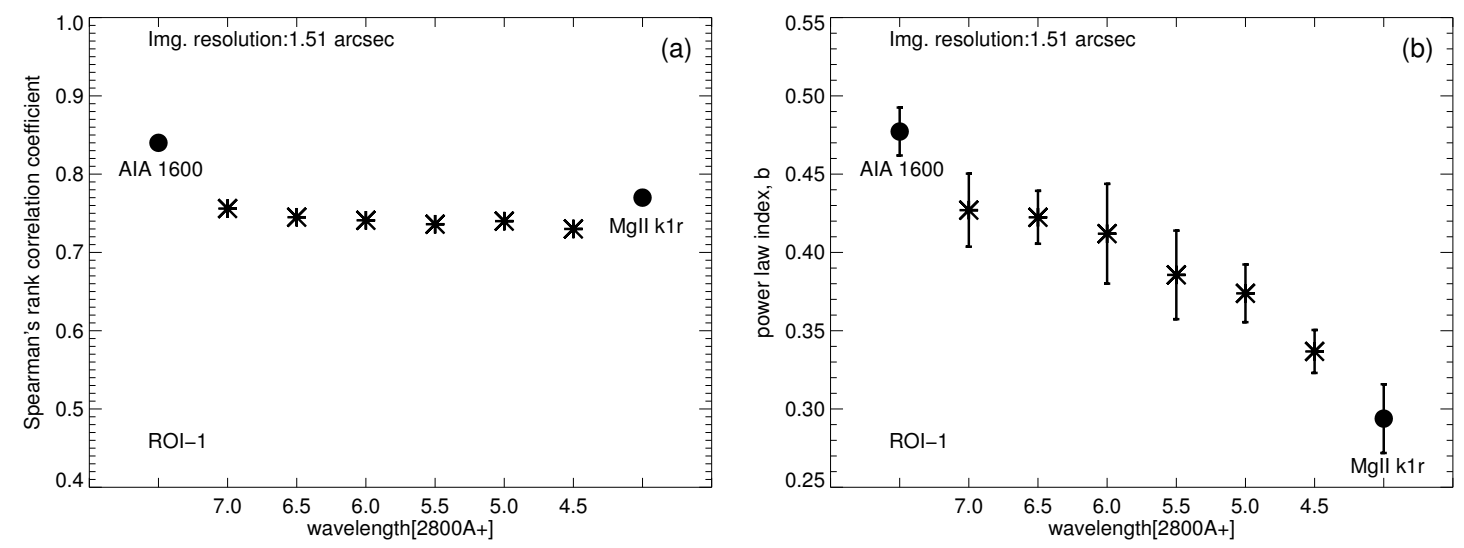

Figure 7.8: Surface magnetic field and emission in the upper photosphere. Using the $\mathrm{Mg}$ II wing this plot fills the gap between the formation region of AIA $1600 \AA$ and Mg II k1r in Figs.7.5 and 7.7. Panel (a) shows the Spearman correlation and panel (b) the power-law index of the relation of the emission to the magnetic field. The asterisks show results based on the emission in $0.5 \AA$ wide bands in the red wing of $\mathrm{Mg}$ II h. The formation height increases with decreasing wavelength (i.e., to the right). These data are based on the active region set with a spatial scale of $1.5^{\prime \prime}$, thus the black filled circles show the same data points as the black filled circles for AIA $1600 \AA$ and Mg II k1r in Figs.7.5 and 7.7. See Sect.7.5.2.

tion that in a coronal hole magnetic fieldlines typically reach less high and are shorter. This could imply a weaker relation of the surface magnetic field to the chromosphere and transition region because more fieldlines close back to the Sun before they reach the chromosphere. Although this might explain the small correlation we see in a coronal hole, clearly further work is needed to better investigate the (magnetic) differences of coronal hole and quiet Sun in the chromosphere and the transition region.

\subsubsection{Mag-flux relation from the photosphere to the chromosphere}

The overall variation of the power-law index of the relation of magnetic field to the emission shows a characteristic hockey-stick shape when plotted with respect to the formation temperature. This effect is present, irrespective of spatial resolution (Fig. 7.5) or region of interest (Fig.7.7). Before discussing the physical cause of the sharp drop from the AIA $1600 \AA$ channel to the Mg II k1r feature we clarify that this drop is real and not just an artefact of the observation, e.g. caused by combining different instruments.

In order to investigate the change from AIA $1600 \AA$ to $\mathrm{Mg}$ II k1r we study the wing of the $\mathrm{Mg}$ II line. According to Vernazza et al.(1981) $\mathrm{k} 1 \mathrm{r}$ forms at the temperature minimum and further out in the wing the emission originates from lower heights in the photosphere (assuming a static atmosphere). Here we investigate the $\mathrm{Mg}$ II wing from $2804.5 \AA$ to $2807.0 \AA$ in $0.5 \AA$ steps, each integrated over a $0.5 \AA$ wide pass band 2 This provides

\footnotetext{
${ }^{2}$ For convenience we look at the red wing of $\mathrm{Mg}$ II h here instead of the k-line used before. The h1r minimum is found at about $2804 \AA$. The used h-line wing is simply because of the location of the line on
} 
information on the upper photosphere with longer wavelengths corresponding to lower heights. We treat the raster maps in these narrow bands in the same way as we treated the other emission features (cf. Sect.7.3.2) and derive the Spearman correlation and the power-law index of the relation to the surface magnetic field. The results are shown in Fig.7.8. Assuming that the AIA $1600 \AA$ channel forms below the Mg II wing (see below) the abscissa in Fig. 7.8 is ordered by formation height (increasing to the right). While Fig.7.8 shows the results for the active region set (at 1.5" spatial scale) the results for the other data sets (and resolutions) are qualitatively the same.

The correlation and power-law indices in the Mg II wing fill the gap between AIA $1600 \AA$ and Mg II k1r quite well. The Spearman correlations of AIA $1600 \AA$ and Mg II k1r to the magnetic field are quite similar (cf. Figs. $7.5 \mathrm{a}, 7.7 \mathrm{a}$ ), and so are the correlation coefficients in the wing of $\mathrm{Mg}$ II (Fig.7.8 a). The main interest is in the variation of the power-law index, and in particular concerning the question if there is a smooth transition on the short end of the hockey stick between AIA $1600 \AA$ and Mg II k1r (cf. Figs.7.5p,7.7b). Here the wing of $\mathrm{Mg}$ II provides a continuous connection emphasising that the power-law index drops smoothly from the upper photosphere to the temperature minimum (Fig. $7.8 \mathrm{p}$ ). This result underlines that the form of the hockey-stick variation is not just a measurement error or an effect of combining different instruments.

This result as depicted in Fig.7.8b motivates two questions: (1) what causes the nonlinearity of the relation between emission and magnetic field in the first place, and (2) why is the power-law index of this relation dropping with height? While the first question has been posed and answered before (e.g. by Schrijver et al.|1989), the second question is new and provides a test for the answer to the first question.

For the non-linear relation between emission and magnetic field Schrijver et al.(1989) gave a simple, mainly geometrical interpretation (their Sect. VII.c). Where the (average) magnetic field is low, the flux tubes emerging from the magnetic concentrations expand freely with height and the chromospheric emission originates at some height of the funnel (or wine glass) shaped magnetic structure. Packing more flux tubes into the same area (i.e. increasing the average field) will first lead to a linear increase of the emission with the magnetic field. Once the packing gets too dense, the expanding flux tubes merge on their way into the upper atmosphere, and for increasing packing (or magnetic field) the height where the flux tubes merge will move down. Thus at a given height the cross-sectional area of each of the flux tubes goes down. Assuming that the chromospheric emission originates always from the same height, this implies that the chromospheric emission cannot keep up with the increase of the magnetic field in a linear fashion. Consequently for higher field strengths the curve in the emission vs. magnetic field plot gets bent (cf. Fig. 7.3), which looks like a power law when plotted on a double-logarithmic plot (cf. Fig.7.10). In a later paper Solanki et al. (1991) employed a two-dimensional magnetostatic model and basically confirmed the qualitative picture of Schrijver et al. (1989).

However, this answer does not yet provide an explanation on how the non-linearity should change throughout the atmosphere with temperature, i.e. the second question outlined above. To address this we first have a closer look at the UV continuum emission.

In the semi-empirical static one-dimensional model of Vernazza et al. (1981), their

the detector within the wavelength window transmitted from the spacecraft: in the case of the red wing of the h-line we can go further into the continuum. The results do not change if we use (a more limited range) with the k-line. 

underlying magnetic field

Fig. 1, the UV continuum near $1600 \AA$ imaged by AIA and the Mg II k1 features should form at the same temperatures, near the temperature minimum. Therefore they should show the same statistical properties when related to the magnetic field, and this is why the significant difference in the power-law index between AIA $1600 \AA$ and Mg II k1r comes as a surprise. However, there is evidence that the EUV continua do not form near the temperature minimum but much lower in the atmosphere.

Relating extreme UV brightenings to the magnetic field, Rouppe van der Voort et al. (2016) wondered why the magnetic concentrations are so well visible in the EUV. In their discussion they argue that this "results also from ionisation of $\mathrm{Fe}$ I and the other neutral 'electron donor' species since their bound-free edges dominate the UV continuous extinction in the upper photosphere." Consequently, the EUV continuum, and also the AIA $1600 \AA$ channel, originate from much lower heights than expected for the Vernazza et al. (1981) type models. Therefore the EUV continuum will have a high contrast with respect to the magnetic field, hence its comparably high power-law index. ${ }^{3}$

Having established that the emission seen in AIA $1600 \AA$ most probably originates from below the $\mathrm{Mg}$ II wing, the question remains if the geometrical explanation of the nonlinearity would be consistent with the observed change of the power-law index with height in the atmosphere. In their original work Schrijver et al. (1989) were only concerned with Ca II, and in their model Solanki et al. (1991) did not discuss how emission forming at different heights would differ in their power-law index. What would the geometrical arguments of Schrijver et al.(1989) predict for two emission features originating at different heights? The higher feature would feel the approach of the two flux tubes earlier, so the effect on the high field strength regions would be more significant. Hence the bending of the relation in the emission vs. magnetic field plot would be stronger, which corresponds to a smaller power-law index. Alternatively one could look at the doublelogarithmic plot where at higher field strengths the effect is stronger and thus the slope of the relation gets more flat, again corresponding to a smaller power-law index. In summary, the geometrical effect suggested by Schrijver et al. (1989) is consistent with the observed decrease of the power-law index from the upper photosphere into the chromosphere as seen in Fig. 7.8 p.

\subsubsection{Mag-flux relation from the chromosphere to the transition re- gion}

From the chromosphere to the transition region the power-law index characterising the relation of the emission to the surface magnetic field increases with formation temperature (Sect.7.4.1.1). Here we will turn to the question how to understand this variation of the long end of the hockey stick in Figs. 7.5 $\mathrm{b}, 7.7 \mathrm{p}$. This is of particular interest because the overall shape remains unchanged irrespective of the spatial resolution or region of interest (while the absolute values of the power-law indices differ). In the previous subsection we gave an interpretation for the short end of the hockey stick, i.e. the drop of the power-law

\footnotetext{
${ }^{3}$ One might also speculate that the power-law index of the AIA $1600 \AA$ channel is higher than for $\mathrm{Mg}$ II because of the contribution of $\mathrm{C}$ IV to the $1600 \AA$ channel. However this is not the case. Firstly, the powerlaw index for Si IV (that should be comparable to C IV) is lower than for the $1600 \AA$ channel. Secondly, we find the same results for the $1700 \AA$ channel that has much less (probably close to none) contamination by C IV.
} 
index from the upper photosphere to the temperature minimum based on the geometrical properties of expanding flux tubes. Obviously that interpretation does not apply for the opposite variation we see through the chromosphere into the transition region.

The increase in power-law index with formation temperature basically reflects that emission forming at higher altitudes will be more sensitive to the magnetic field. Considering that the X-ray emission clearly shows a very high contrast to the magnetic field, it seems natural to find this increasing sensitivity to the magnetic field already in the chromosphere and transition region. Here the magnetic field starts dominating the plasma (plasma- $\beta$ is smaller than unity) and the magnetic heating has to take over from acoustic heating, because the latter is not sufficient to heat the higher regions of the atmosphere. This interpretation is also backed by studies of the flux-flux relations from the chromosphere to the corona of the Sun (Schrijver 1992) and in other stars (Schrijver 1987).

This provides some insight into which processes govern the relation of the emission in the upper atmosphere to the magnetic field. In their qualitative considerations Schrijver et al. (1989) speculated in their Sect. VII.c that the non-linear relation of emission and magnetic field "can be explained as a combined effect of geometrical filling of (...) flux tubes [and] the dependence of the heating efficiency on flux-tube packing". In our study, where we also investigate the temperature dependence of this non-linearity we can be more specific. To be consistent with the hockey-stick type temperature variation, from the upper photosphere into the temperature minimum region the geometrical effects (discussed in Sect.77.5.2) would dominate, while from the chromosphere into the transition region and probably the corona the increasing sensitivity of the emission to the magnetic heating processes becomes dominant.

\subsubsection{Mag-flux relation at different resolutions and regions}

The results discussed above for the active region set at $1.5^{\prime \prime}$ resolution basically holds also for the other regions of interest and at different spatial resolutions. In the following we give some explanations where the mag-flux relations differ from the $1.5^{\prime \prime}$ active region set.

The slight increase of the power-law indices for lower spatial resolution (cf. Fig. $7.5 \mathrm{~b}$ ) most probably is a consequence of the basal flux. When degrading the data spatially we find that the basal flux increases slightly. This is because after the degradation, regions of formerly high magnetic field (and thus high emission) will have lower magnetic flux densities (in particular if close-by opposite polarities cancel). These areas are then included in the mask to calculate the basal flux (cf. Sect.7.3.1) resulting in a larger basal flux. Figuratively speaking, subtracting a larger basal flux pulls down the emission at low magnetic fields more strongly (in the double-logarithmic plot) and thus the power-law fit gives a steeper slope, viz. larger power-law index.

Of the regions under investigation, the plage region sticks out in terms of the overall level of the power-law index: the hockey stick for the plage region is well below the other areas (Sect.7.4.2, Fig.7.7p). This is mainly an artefact of the definition of the plage region. Because this excludes the darker quiet Sun parts (cf. Sect.7.2.3,2), here we basically cut the lower-intensity region of the scatter plot of the intensity vs. magnetic field scatter plots (e.g. Fig.7.3). Consequently, the variation over the considered range of magnetic field values will be smaller and the derived power-law indices will be smaller. 
7 Emission of solar chromospheric and transition region features related to the underlying magnetic field

For the quiet Sun and coronal hole regions the situation is different. While the correlations for these two regions are smaller than for the active region (just as the plage), the power-law indices are actually comparable (cf. Fig. 7.7). However, unlike in the plage regions, for the quiet Sun and coronal hole regions there is no cut-off at low intensities and hence the power-law indices are comparable to the active region.

\subsubsection{Flux-flux relations: the Sun and stars}

The relation between the radiative fluxes between different emission features provides a good characterisation for the interaction between different parts of the atmosphere and their different response to the underlying magnetic field. In particular if magnetic measurements are not available (like in many cases of stellar observations) the flux-flux relations provide a valuable tool. For the Sun, observations of the surface magnetic field and the emission from the upper atmosphere are acquired by different instruments, which introduces unavoidable uncertainties concerning the spatial (and temporal) alignment. However, when using spectroscopic observations with different emission features through the same spectrograph slit, the resulting raster maps provide image information that is exactly co-spatial and co-temporal. This is one of the major advantages when relating the IRIS data in the features of $\mathrm{Mg}$ II, $\mathrm{C}$ II and $\mathrm{Si}$ IV, as done in Sect.7.4.1.2.

Relating each emission feature to the emission forming at the lowest temperature (Mg II k1r) we find that the power law-index increases with formation temperature (cf. Table 7.4, Sect.7.3.3.2. Of course, this basically reflects the relations between (unsigned) magnetic field and emission as discussed above. Our finding is also consistent with Bennett et al. (1984), Cappelli et al. (1989) and Schrijver (1992), all of whom noticed that the emission from the transition region grows more rapidly than that from the chromosphere.

The steepening of the flux-flux relations with temperature we find here for spatially resolved data from the Sun is also found when using stellar data. For example, analysing a number of G stars Ayres et al. (1981) showed that the flux-flux relation has a steeper gradient going from the chromosphere into the corona. In the stellar case some evidence has been presented that there is a clear distinction between the chromosphere and the transition region in terms of flux-flux relations. In particular, Oranje (1986) found a separation of the power-law index of the flux-flux relations between the chromospheric and the transition region lines for F-G type stars: while the power-law index of the chromospheric Si II line to $\mathrm{Mg}$ II is only 1.2, there is a jump to the transition region lines such as C II or Si IV with a power-law index of about 1.6 (Oranje 1986, Fig. 7). However, our analysis does not show this jump (or separation) we find a continuous change with formation temperature, in fact, we find only a small difference between $\mathrm{Mg}$ II k3 and C II. Maybe Oranje (1986) found this jump only because he was not investigating the different features of the Mg II line as we do here. Also, considering the physical scenario to understand the steepening of the power-law indices from the chromosphere into the corona discussed above (Sect.7.5.3) would not provide an explanation for a jump. So if this jump in stellar observations is real, the reason for this would have to be found in some global properties of the atmosphere when integrating over the whole stellar disk, but not in the individual processes governing the physics of the individual magnetic flux tubes connecting the photosphere to the upper atmosphere.

While the power-law indices we derived mostly do not depend on spatial resolu- 
tion (cf. Sect.7.5.4), there is one noticeable exception: the flux-flux relation of Si IV to $\mathrm{Mg}$ II k1r. Here we see higher power-law indices for higher resolution (Fig.7.6). At this point we can only speculate on this, because further observational confirmation would be needed for this finding. It might well be that at smaller spatial scales Si Iv becomes more dependent on the magnetic field. This could point to the (expected) feature that the magnetic heating operates at the smallest scales we can observe, and probably even below that.

Comparing different flux-flux relations we find that the scatter in the emission (in the flux-flux relation) has to be independent of the emission itself (Sect.77.4.1.2). This could be consistent with a scenario where the underlying magnetic field governs the upper atmosphere in independent ways. For example, different spatial scales of the (opposite directed) magnetic flux concentrations in the photosphere might lead to an energisation of different atmospheric heights, hence temperature regimes. So one would statistically expect that the chromosphere and the transition region brighten at higher magnetic field strengths, but the details, i.e. if the chromospheric or transition region plasma gets brighter, might change from case to case, which then causes the considerable scatter (being independent of the actual emission). This would also be consistent with the analysis of times series data comparing the chromosphere (EUV continuum) to the lower (C II) and upper transition region (O vI) as reported by Brković and Peter (2003). Here sometimes the brightenings in all three spectral features are co-temporal, sometimes only two features brighten up, sometimes the brightening in one feature comes alone: no particular pattern could be established observationally.

\subsection{Conclusion}

We investigated the connection between emission originating from different temperatures from the upper solar atmosphere to each other and to the underlying magnetic field. The IRIS spectroscopic maps provide not only unprecedented spatial resolution, but more importantly a continuous coverage from the photosphere through the chromosphere into the transition region to the corona. The wings of $\mathrm{Mg}$ II, the spectral features of the $\mathrm{Mg}$ II line core, and $\mathrm{C}$ II and $\mathrm{Si}$ IV are observed with the same spectrograph which eliminates any issues of alignment.

As expected for a magnetic field expanding into the upper atmosphere we confirm the continuous decrease of the correlation coefficient between emission and photospheric magnetic field with increasing line formation temperature. As a new result we found that this continuous decrease is also present through the spectral features of the $\mathrm{Mg}$ II line, i.e. from $\mathrm{k} 1 \mathrm{r}$ through $\mathrm{k} 2 \mathrm{r}$ to $\mathrm{k} 3$ that form at increasing temperature through the chromosphere according to semi-empirical (static) models. Considering the highly dynamic and complex structure of the chromosphere (e.g. Wedemeyer-Böhm et al. 2009) this is surprising.

The main result of this study is the change of the power-law index of the relation between the emission and the magnetic field that follows a power law. Here we find a hokey-stick like variation. From the photosphere to the temperature minimum the powerlaw index drops quickly and then rises again through the chromosphere into the transition region (Figs.7.5p and 7.7p). Considering the concept of Schrijver et al. (1989) to understand the non-linear relation between the chromospheric emission and the magnetic field 
7 Emission of solar chromospheric and transition region features related to the underlying magnetic field
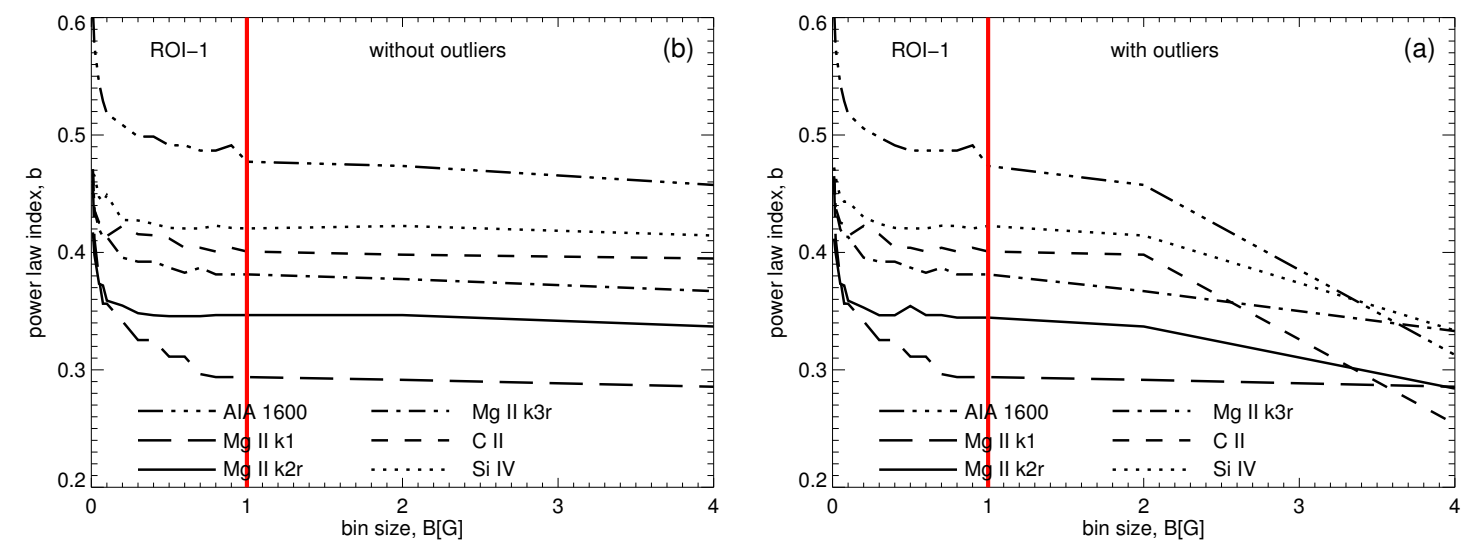

Figure 7.9: Power-law relation and basal flux. The left panel (a) presents the power-lawindex $b$ between magnetic field and the emission flux proxies for different values of the basal flux determined in bins of different sizes (for all data points with magnetic field strengths below $4 \mathrm{G}$ ). For larger bin-sizes the basal flux, and thus the derived power law index is constant. The right panel (b) shows the same, but for data sets where we added outliers to the data. The vertical red lines indicates the bin size we use in our study. See Appendix 7.7.1.

we found that this would also offer an explanation for the drop of the power-law index from the photosphere to the temperature minimum. Basically this is a geometrical effect related to the merging of expanding magnetic flux tubes rooted in the photosphere. The increase of the power-law index with temperature in the chromosphere and transition region would not be consistent with this geometrical scenario, but is based on an entirely different effect, most probably related to the sensitivity of the emission from the upper atmosphere to the magnetic heating process.

\subsection{Appendix}

\subsubsection{Basal flux calculation}

To investigate the relation between the magnetic field strength, $|B|$, and the flux in an emission line or continuum, $I$, one has to determine the basal flux. In Sect.7.3.1 we briefly discussed the method we employ to calculate the basal flux. In this appendix we give some further details on the basal flux determination and in particular discuss what impact this has on the power law index characterising the relation between $|B|$ and $I$.

As outlined in Sect.7.3.1, in their original work Schrijver et al. (1989) defined the basal flux as the component of emission which originates independently of the magnetic field from acoustic processes, e.g., the heating of the chromosphere through the dissipation of sound waves that steepen into shocks. To determine the basal flux we concentrate on the non-magnetic regions in the respective region-of-interest, i.e. we consider only spatial pixels where the signal of the magnetogram is consistent with noise. In our study we use HMI which has a noise level of about 5-10 G (J. Schou, priv. comm.). To be on the 
safe side concentrating on nearly-field-free regions, we use only half of the noise level as the upper limit for the magnetic field strength, i.e. $4 \mathrm{G}$.

Because of the limited spatial resolution and polarimetric sensitivity these nearlyfield-free regions still might host significant magnetic patches, simply hidden to the instrument. Thus one cannot simply take e.g. the mean or median value of the intensity distribution in these areas with low magnetic field (below 4G) - this would give a too high value of the basal flux because most probably one would include quite a few regions with hidden magnetic field that boost the emission. Instead one should still follow the concept of Schrijver et al. (1989) and find the minimum value of the emission.

If one would simply define the minimum of the intensity in all the regions with a magnetic field strength below a threshold $(4 \mathrm{G})$ one would be sensitive to outliers. For example, if there are only a few pixels with very small intensity values (for whatever reason) these would result in a gross underestimation of the basal flux. To avoid this problem of outliers one could consider to define the basal flux based on the distribution of the intensity in these regions of low magnetic signal (below $4 \mathrm{G}$ ). For example, one could define the basal flux at the 5 or 10 percentile level of the distribution. Or one could use the width of the distribution of intensities to define the basal flux, e.g., the mean value minus the standard deviation of the intensities. However these definitions rely on the bulk part of the distribution of intensity values. Thus they are not well suited to define the basal flux, i.e., a minimum value of intensities. Still, we tested this, but as expected we found that the basal flux defined through a percentile or the standard deviation would be very sensitive to the choice of parameters (e.g., 5th or 10th percentile, one or two standard deviations). Therefore these strategies are not a good procedure to determine the basal flux without sensitivity to outliers.

To overcome the sensitivity to outliers we subdivide the regions below a threshold (of $4 \mathrm{G}$ ) into bins with respect to the magnetic field. We choose to have bins linearly spaced in magnetic field strength. In each of these bins we determine the minimum intensity and then define the median value of these minimum values in the bins as the basal flux.

To test our method and to determine the optimum size for the bins in magnetic field strength, we calculate the basal flux for a number of different bin sizes. In the end we want to get reliable results for the power law index of the relation between the magnetic field and intensity (Sect.7.3.2.2). Thus we used the basal fluxes determined from our procedure with different bin sizes, corrected the intensities for the respective basal flux and calculate the power law index for each of the bin sizes. The results are shown in Fig. 7.9. We concentrate first on the left panel that shows a data set without outliers. As expected for small bin sizes we get too large basal fluxes that result in steeper power laws (i.e. larger power law indices $b$ ). Basically, when the bin size is too small there will be only a few data points in each bin and thus, statistically speaking, the mean minimum intensity in each bin will not be too different from the mean in the same bin. Then the median of the minima in the bins, i.e. the basal flux as we define it, will be close to the mean value of the distribution of all intensities below the magnetic field threshold (of $4 \mathrm{G})$. Consequently too small bin sizes overestimate the basal flux which also leads to too large power-law-indices. From the left panel of Fig.7.9 we conclude that the bin size should be about $0.7 \mathrm{G}$ or larger. For larger bin sizes the derived power-law-indices for the different emission proxies remain roughly constant.

To check that our method works also in the presence of outliers we took one data 
set without outliers and added extra data points to each analysed intensity channel with low intensity to mimic outliers. We apply the same procedure as before and calculate the power-law index as a function of bin size for different emission proxies and show the result in the right panel of Fig.7.9. The largest bin size shown in the plot, $4 \mathrm{G}$, implies that there is only one bin, i.e. the basal flux is the minimum of all pixels below the threshold of magnetic field strength, just as in the original method by Schrijver et al.(1989). We see that for too large bins the basal flux and hence the power-law-index becomes sensitive to the outliers resulting in too small power-law indices. Still, this effect becomes significant only for bin sizes above about $2 \mathrm{G}$. Of course, the exact values of the optimum bin sizes will depend on the data set used and the number of data points considered in the analysis.

For our data set we find that with a bin size of about $1 \mathrm{G}$ we get reliable and robust results for the power-law-index characterising the relation between magnetic field and photon flux in different emission proxies, i.e. the AIA $1600 \AA$ continuum channel, the features of the chromospheric $\mathrm{Mg}$ II line, and the transition region lines of $\mathrm{C}$ II and $\mathrm{Si}$ IV. Our method extends the original procedure by Schrijver et al. (1989) allowing to cope with outliers and at the same time avoids the sensitivity to the choice of parameters when using percentiles of the intensity distribution or the standard deviation of the intensities to calculate the basal flux.

\subsubsection{Comparing different methods to determine power-law relations}

In Sect.7.3.2.2 we discussed two methods (below labeled I and II) to determine the powerlaw index $b$ of the non-linear relation between emission proxies and the underlying magnetic field. There we imaged the results in a linear plot in Fig.7.3. Here we show the same data but on a double-logarithmic scale in Fig.7.10.

To check the reliability of obtained the power-law fits, we used several methods and applied them to the same data points. In the following we recall the two methods discussed in Sect.7.3.2.2 (I and II) and briefly explain three further methods (III-V) to derive the power-law indices.

(I) Least-squares fit to original data points. After subtracting the basal flux $I_{0}$ from the intensities $I$ giving $I^{\prime}=I-I_{0}$ (Appendix 7.7.1) we fit a power law, $I^{\prime}=a|B|^{b}$.

(II) Least-squares fit to binned data. We first collect the data points into bins with respect to the magnetic field strength and compute the average value of the intensity in each bin (bars in Fig. 7.3) and then perform the power-law fit to these averages.

(III) Variant of Method I with different basal flux. As discussed in Appendix 7.7.1, to compute the basal flux $I_{0}$ we take the median value of the minimum intensities in bins below a magnetic field threshold $(4 \mathrm{G})$. To go to the extremes of basal fluxes, we also take the minimum, $I_{0}^{(\min )}$, and the maximum values, $I_{0}^{(\max )}$, of the intensities below the threshold. For these three basal fluxes we perform the power-law fit and derive three (different) values for the power-law index. In Fig.7.11 we plot the mean value of the three indices.

(IV) Linear fit to logarithmic data values. This is similar to method I, but using a linear least-squares fit to the data points on a logarithmic scale, i.e. $\left(\log I^{\prime}\right)=b(\log |B|)+\tilde{a}$. This should be equivalent to the power-law fit to the original data, of course, with the slope of the linear fit taking the role of the power-law index.

(V) Ellipse fitting of the logarithmic data values. In this method we fit ellipses to the 

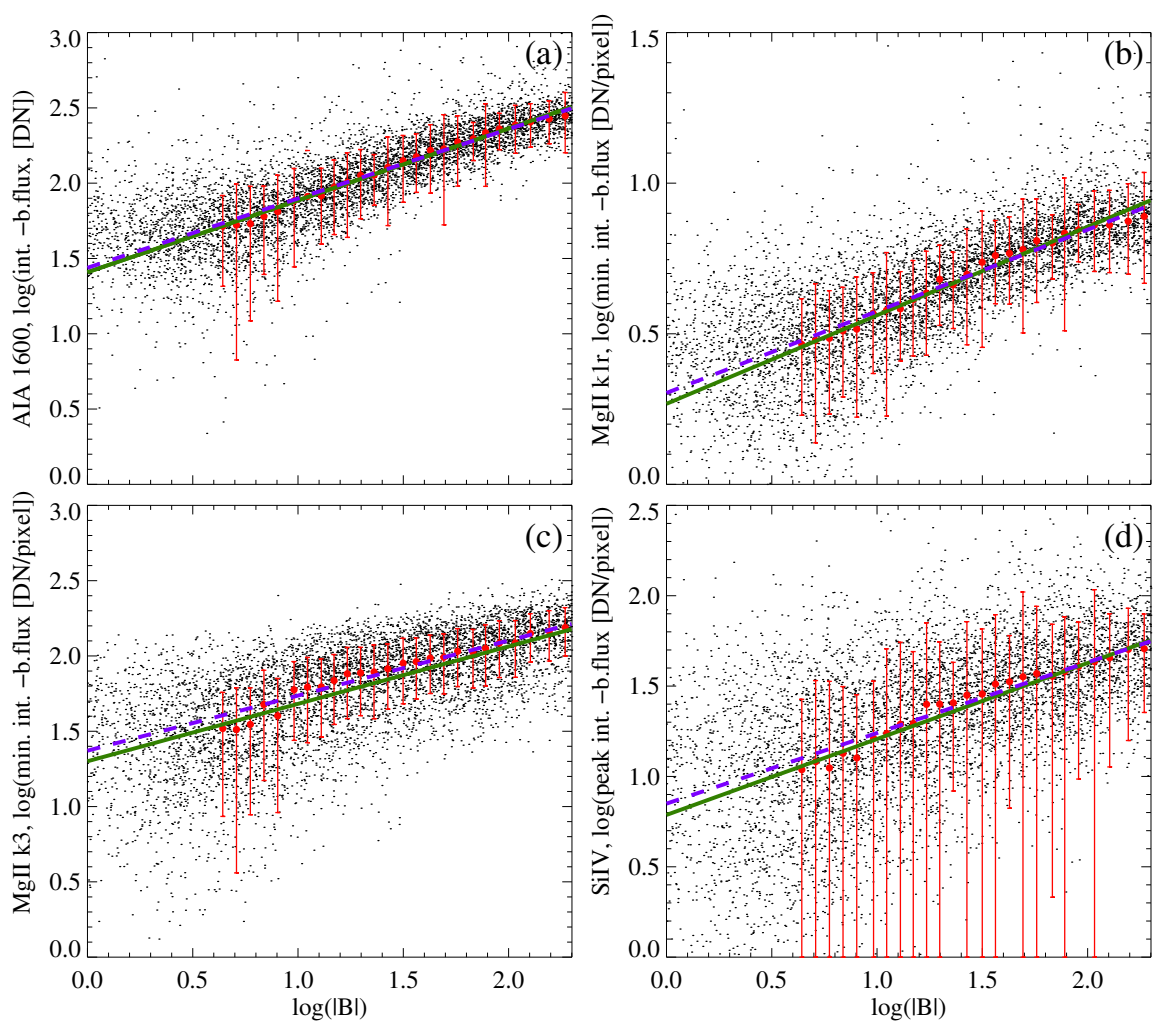

Figure 7.10: Relation of the upper atmosphere emission to the underlying magnetic field. This shows the same data as Fig.7.3, but now on a double-logarithmic scale to emphasise the power-law nature of the relations. Here the basal flux (b.flux) in the respective emission feature is subtracted. See Sect.7.3.2.2 and Appendix 7.7.2.

(2D) probability distribution functions, i.e. the point density of the scatter plots $\log I^{\prime}$ vs. $\log |B|$, as shown in Fig.7.10. This follows the procedure as described in Sect.7.3.3.2 to derive the power-law indices of the flux-flux relations.

These methods agree well with respect to the power-law indices. In Fig.7.11 we show the power-law indices derived by the five methods for the various emission features ordered by formation temperature. In particular all the five methods show the hockey-stick feature (cf. Figs.7.5b and 7.7b; Sect.7.4.1.1). Therefore this feature does not depend on the method employed and the discussion on the physical implications of this in Sects.7.5.2 and 7.5.3 is based on a solid observational footing. 
7 Emission of solar chromospheric and transition region features related to the underlying magnetic field

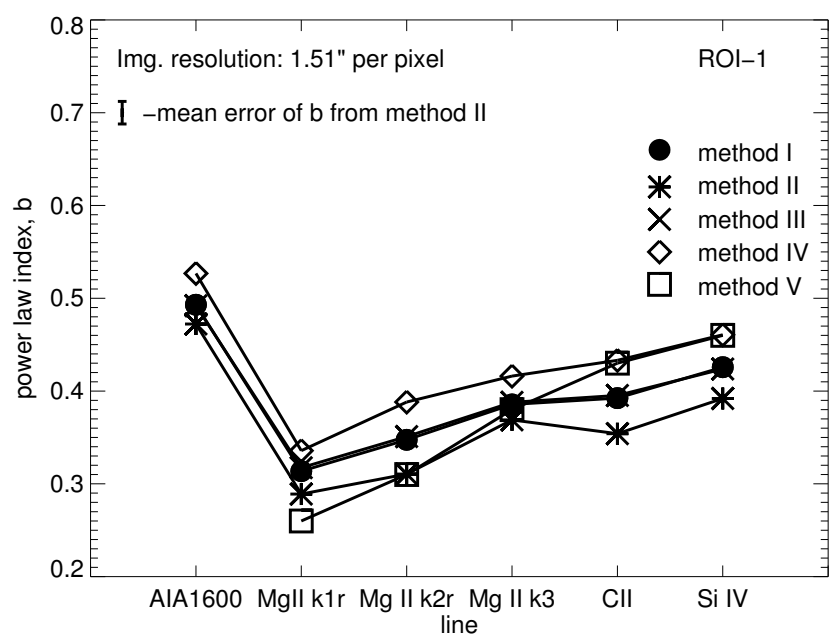

Figure 7.11: Comparison of power-law indices derived from five methods. This plot shows the power-law indices of the relation of intensity vs. magnetic field for the various emission features in the same way as displayed in Figs. $7.5 \mathrm{~b}$ and $7.7 \mathrm{~b}$. The five methods are briefly described in Appendix 7.7.2. 


\section{Summary and outlook}

In this work, we have studied the properties of small-scale structures in the upper solar atmosphere. For this, we used observational data acquired by SDO, Hinode, IRIS and Hi-C.

As a first step, we investigated the geometrical and thermal properties of small-scale loop-like structures observed in a plage region at coronal temperatures $(>1 \mathrm{MK})$, by Hi-C and SDO. Peter et al. (2013) found four of them in the Hi-C images, but the nature of these remained open. We consider three scenarios which can explain the origin of these: (1) small-scale loops with granular size, (2) elongated moss type structures (as one of the footpoints of a hot coronal loop) heated from the corona, and (3) elongated structures heated from below. To find the most likely scenario, we studied the size, motion and temporal evolution of these features. We found that small-scale loop-like structures have a length of approximately $1 \mathrm{Mm}$ and a lifetime of at least $100 \mathrm{~s}$. The centers of the structures move in random directions with an average velocity of about $5 \mathrm{~km} \mathrm{~s}^{-1}$. These results are consistent with photospheric motions, so we suggest that the photospheric magnetic field can plays an important role in the evolution of these structures. Therefore, we decided to investigate their relation to the underlying photospheric magnetic field. We found a region with unipolar magnetic field hosting small-scale structures. Still, we suggest that the small-scale structures are related to bipolar structures in the photosphere, only that we cannot resolve these with current instruments. Therefore, to determine the most probable scenario, we investigate differential emission measure (DEM) to study the thermal properties of the small-scale loop-like structures. We found significant differences of DEM(T) between the plage region hosting the small loop-like features and moss region. Such moss areas show small patches of $1 \mathrm{MK}$ plasma a at the footpoint regions of hot loop reaching well above $5 \mathrm{MK}$. Both moss and small loop structures have a peak of DEM(T) at $\log T \approx 6.2$. However, for the moss, we found a second peak of $\mathrm{DEM}(\mathrm{T})$ at $\log T \approx 6.6$ and a significantly lower emission from the small-scale loop-like structure at the same temperature. Additionally, in X-ray observations provided by XRT/Hinode and EUV data in AIA94 $\AA$ we did not find emission in the temperature range from $2 \mathrm{MK}$ to $10 \mathrm{MK}$, a temperature range which is characteristic of hot loops. Therefore, the small-scale structures cannot be moss. To check the third scenario, we conduct a Gedanken experiment. In this scenario, we assume that small-scale loop-like structures exist at the footpoints of long diluted loops which are heated from below. We assume that the plasma in the hotter part of the loop is not dense enough to be a source of X-ray emission. However, this thermal and density stratification suggests that the dense plasma at $\log T \approx 6.2$ (the pressure height scale height is approximately $75 \mathrm{Mm}$ ) should expand into the upper part of the solar atmosphere with the adiabatic sound speed (approximately $185 \mathrm{~km} \mathrm{~s}^{-1}$ at $\log T=6.2$ ). Given the lifetime of the structure (100s) the expanding structure should become significantly 
larger than the size of the small-loop-like features we observe. Therefore, we exclude the third scenario and suggest that small-scale loop-like structures are a small-scale version of the hot coronal loops, like miniature loops. This structure can be related to the small-scale flux tubes emerging from the photosphere studied by Ishikawa et al. (2010). Small-scale loops $(1 \mathrm{Mm})$ at transition region temperatures have been also investigated by Hansteen et al. (2014). Therefore, we suggest that they can emerge to greater heights and exist even at coronal temperatures. Further studies should find an answer of how often and under which conditions these phenomena occur in the solar corona.

Next, we investigated the temporal and spatial evolution of the small-scale structures observed in a quiet Sun area of the transition region at high spatial cadence. This study focuses on the relation between the emission structures and the underlying magnetic field. We found that the small-scale loop-like structure is connected two opposite magnetic field polarities. This structure shows intense emission in Si IV and is almost invisible in observations showing the temperature minimum. Therefore, we suggest one scenario where magnetic field emerges from the lower part of the solar atmosphere. The reconnection occurs in chromosphere or in the transition region causing strong heating which is a source of the emission observed in the Si IV. In another region-of-interest we identified two magnetic field patches with a similar magnetic field strength and flux, but with different EUV emission associated with them. These similarities as well as the similar temporal and spatial evolution, and close location of the magnetic patches suggests a similar morphology and origin of these structures. We suggest these structures are related to a convective collapse. The difference of the emission of the two patches may be a result of their different inclination. Based on these studies, we conclude that similar small-scale magnetic field configurations can result in significantly different UV emission. Also the large scale magnetic field structures will have an important influence on the small-scale structures.

As the last step, we investigated the relation between the emission from the solar chromosphere and transition region to the underlying magnetic field. Small-scale structures are abundant in the chromosphere and transition region. However, even using modern instruments, not all small-scale structures are visible. Therefore, we use a statistical method to understand how the unresolved structures may influence the solar atmosphere. Using spectral maps of $\mathrm{Mg}$ II, C II, and Si IV obtained by IRIS and the line-of-sight photospheric magnetic field from HMI we investigated the spatial connection between emission from different atmospheric layers and their relation to the underlying magnetic field. We have analyse a different types of regions: active region, plage, quiet sun, and coronal hole. First, we analysed the correlation between magnetic field and emission. To this end, we subtract the component of the emission which is independent of the magnetic field (called the basal flux). The relation between magnetic field and emission is non-linear, therefore we use the Spearman's correlation coefficient. This shows that the correlation between magnetic field and emission monotonically decreases with line formation temperature for all regions. This trend is independent of spatial resolution. This trend is consistent with the trend of the correlation coefficient for the mutual flux-flux analysis. We assume a power-law relation between magnetic field and emission and use the power-law index to characterise this dependence. This variation has a hokey-stick shape. The power-law index decreases from the photosphere to the temperature minimum and increases through the chromosphere into the transition region. Also this trend is independent of spatial resolution and the choice of region-of-interest. It is consistent with the trend of the power- 
law-indices for the mutual flux-flux relations. Based on these results we conclude that geometrical effects of the expanding plasma tubes govern the relation between the photospheric emission and magnetic field. Above the temperature minimum, the magnetic heating plays an important role. As we move higher into the atmosphere, the emission becomes more sensitive to the magnetic field, so the power-law index also increases with temperature. This study shows for the first time that the power-law-index increases continuously above the temperature minimum. This result, as well as the flux-flux relations, provide useful tools to study solar or stellar atmospheres especially when measurements of the magnetic field are not available.

\section{Outlook}

In this work, we analysed the properties of miniature hot loops, moss, transition region small loop-like structures and convective collapse. However, the zoo of the small-scale structures is much more abundant. These structures can have similar properties (size, life time), but also can show differences (evolution, relation to the magnetic field). Further work about small-scale structures should address radiative losses for the different types of small-scale structures based on a statistical study. It is a starting point to investigate the impact of these structures have on coronal heating. For this, a classification of small-scale structures based on morphology, evolution, and relation to the magnetic field is needed. It will be a useful tool for the identification of the small-scale structures as well as to study their properties. To this end, more simultaneous observations of the EUV intensity and the magnetic field with high-spatial and temporal resolution are needed.

The study of the mag-flux and flux-flux relations shows that the EUV intensity can be use as a proxy of solar activity. This study should be extended to more regions-of-interests to obtain better statistics of the power-law-indices. It will be also interesting to compare the mag-flux and flux-flux analysis as presented in this thesis to results of 3D simulations of the corona of the Sun. 



\section{Bibliography}

Alfvén, H., 1947, Magneto hydrodynamic waves, and the heating of the solar corona, MNRAS, 107, 211

Anders, E., Grevesse, N., 1989, Abundances of the elements - Meteoritic and solar, Geochim. Cosmochim. Acta, 53, 197-214

Aschwanden, M. J., 2004, Physics of the Solar Corona. An Introduction, Praxis Publishing Ltd

Asplund, M., Grevesse, N., Sauval, A. J., Scott, P., 2009, The Chemical Composition of the Sun, ARA\&A, 47, 481-522, 0909.0948

Ayres, T. R., Marstad, N. C., Linsky, J. L., 1981, Outer atmospheres of cool stars. IX - A survey of ultraviolet emission from F-K dwarfs and giants with IUE, ApJ, 247, $545-559$

Baliunas, S. L., Donahue, R. A., Soon, W. H., Horne, J. H., Frazer, J., Woodard-Eklund, L., Bradford, M., Rao, L. M., Wilson, O. C., Zhang, Q., Bennett, W., Briggs, J., Carroll, S. M., Duncan, D. K., Figueroa, D., Lanning, H. H., Misch, T., Mueller, J., Noyes, R. W., Poppe, D., Porter, A. C., Robinson, C. R., Russell, J., Shelton, J. C., Soyumer, T., Vaughan, A. H., Whitney, J. H., 1995, Chromospheric variations in main-sequence stars, ApJ, 438, 269-287

Barczynski, K., Peter, H., Savage, S. L., 2017, Miniature loops in the solar corona, A\&A, 599, A137, 1611.08513

Bennett, J. O., Ayres, T. R., Rottman, G. J., 1984, The solar stellar connection in the far ultraviolet, in NASA Conference Publication, (Eds.) J. M. Mead, R. D. Chapman, Y. Kondo, vol. 2349 of NASA Conference Publication

Berger, T. E., De Pontieu, B., Schrijver, C. J., Title, A. M., 1999, High-resolution Imaging of the Solar Chromosphere/Corona Transition Region, ApJ, 519, L97-L100

Bhatnagar, A., Livingston, W., 2005, Fundamentals of Solar Astronomy

Biermann, L., 1948, Über die Ursache der chromosphärischen Turbulenz und des UVExzesses der Sonnenstrahlung, ZAp, 25, 161

Boerner, P., Edwards, C., Lemen, J., Rausch, A., Schrijver, C., Shine, R., Shing, L., Stern, R., Tarbell, T., Title, A., Wolfson, C. J., Soufli, R., Spiller, E., Gullikson, E., 
McKenzie, D., Windt, D., Golub, L., Podgorski, W., Testa, P., Weber, M., 2012, Initial Calibration of the Atmospheric Imaging Assembly (AIA) on the Solar Dynamics Observatory (SDO), Sol. Phys., 275, 41-66

Bray, R. J., Cram, L. E., Durrant, C., Loughhead, R. E., 1991, Plasma Loops in the Solar Corona

Brković, A., Peter, H., 2003, Relation of transition region blinkers to the low chromosphere, A\&A, 406, 363-371

Brown, D. S., Parnell, C. E., Deluca, E. E., Golub, L., McMullen, R. A., 2001, The Magnetic Structure of a Coronal X-Ray Bright Point, Sol. Phys., 201, 305-321

Cappelli, A., Cerruti-Sola, M., Pallavicini, R., Cheng, C. C., 1989, A comparison of solar and stellar ultraviolet spectra obtained with SKYLAB and IUE, A\&A, 213, 226-244

Centeno, R., Socas-Navarro, H., Lites, B., Kubo, M., Frank, Z., Shine, R., Tarbell, T., Title, A., Ichimoto, K., Tsuneta, S., Katsukawa, Y., Suematsu, Y., Shimizu, T., Nagata, S., 2007, Emergence of Small-Scale Magnetic Loops in the Quiet-Sun Internetwork, ApJ, 666, L137-L140, 0708.0844

Cheung, M. C. M., Boerner, P., Schrijver, C. J., Testa, P., Chen, F., Peter, H., Malanushenko, A., 2015, Thermal Diagnostics with the Atmospheric Imaging Assembly on board the Solar Dynamics Observatory: A Validated Method for Differential Emission Measure Inversions, ApJ, 807, 143, 1504.03258

Chitta, L. P., Peter, H., Solanki, S. K., Barthol, P., Gandorfer, A., Gizon, L., Hirzberger, J., Riethmueller, T. L., van Noort, M., Blanco Rodriguez, J., Del Toro Iniesta, J. C., Orozco Suarez, D., Schmidt, W., Martinez Pillet, V., Knoelker, M., 2016, Solar coronal loops associated with small-scale mixed polarity surface magnetic fields, ArXiv e-prints, 1610.07484

Cirtain, J. W., Golub, L., Winebarger, A. R., de Pontieu, B., Kobayashi, K., Moore, R. L., Walsh, R. W., Korreck, K. E., Weber, M., McCauley, P., Title, A., Kuzin, S., Deforest, C. E., 2013, Energy release in the solar corona from spatially resolved magnetic braids, Nature, 493, 501-503

Copernicus, N., 1543, De revolutionibus orbium coelestium

De Pontieu, B., Title, A. M., Lemen, J. R., Kushner, G. D., Akin, D. J., Allard, B., Berger, T., Boerner, P., Cheung, M., Chou, C., Drake, J. F., Duncan, D. W., Freeland, S., Heyman, G. F., Hoffman, C., Hurlburt, N. E., Lindgren, R. W., Mathur, D., Rehse, R., Sabolish, D., Seguin, R., Schrijver, C. J., Tarbell, T. D., Wülser, J.-P., Wolfson, C. J., Yanari, C., Mudge, J., Nguyen-Phuc, N., Timmons, R., van Bezooijen, R., Weingrod, I., Brookner, R., Butcher, G., Dougherty, B., Eder, J., Knagenhjelm, V., Larsen, S., Mansir, D., Phan, L., Boyle, P., Cheimets, P. N., DeLuca, E. E., Golub, L., Gates, R., Hertz, E., McKillop, S., Park, S., Perry, T., Podgorski, W. A., Reeves, K., Saar, S., Testa, P., Tian, H., Weber, M., Dunn, C., Eccles, S., Jaeggli, S. A., Kankelborg, C. C., Mashburn, K., Pust, N., Springer, L., Carvalho, R., Kleint, L., Marmie, J., Mazmanian, E., 
Pereira, T. M. D., Sawyer, S., Strong, J., Worden, S. P., Carlsson, M., Hansteen, V. H., Leenaarts, J., Wiesmann, M., Aloise, J., Chu, K.-C., Bush, R. I., Scherrer, P. H., Brekke, P., Martinez-Sykora, J., Lites, B. W., McIntosh, S. W., Uitenbroek, H., Okamoto, T. J., Gummin, M. A., Auker, G., Jerram, P., Pool, P., Waltham, N., 2014, The Interface Region Imaging Spectrograph (IRIS), Sol. Phys., 289, 2733-2779, 1401.2491

Dere, K. P., 2008, The plasma filling factor of coronal bright points. Coronal bright points, A\&A, 491, 561-566

Dowdy, Jr., J. F., 1993, Observational evidence for hotter transition region loops within the supergranular network, ApJ, 411, 406-409

Dravins, D., 1975, Horizontal velocities in the solar photosphere, Sol. Phys., 40, 53-63

Edlén, B., 1943, Die Deutung der Emissionslinien im Spektrum der Sonnenkorona. Mit 6 Abbildungen., ZAp, 22, 30

Feldman, U., 1983, On the unresolved fine structures of the solar atmosphere in the 30,000-200,000 K temperature region, ApJ, 275, 367-373

Fuller, W. A., 1995, Introduction to Statistical Time Series

Golub, L., Krieger, A. S., Silk, J. K., Timothy, A. F., Vaiana, G. S., 1974, Solar X-Ray Bright Points, ApJ, 189, L93

Golub, L., Deluca, E., Austin, G., Bookbinder, J., Caldwell, D., Cheimets, P., Cirtain, J., Cosmo, M., Reid, P., Sette, A., Weber, M., Sakao, T., Kano, R., Shibasaki, K., Hara, H., Tsuneta, S., Kumagai, K., Tamura, T., Shimojo, M., McCracken, J., Carpenter, J., Haight, H., Siler, R., Wright, E., Tucker, J., Rutledge, H., Barbera, M., Peres, G., Varisco, S., 2007, The X-Ray Telescope (XRT) for the Hinode Mission, Sol. Phys., $243,63-86$

Grotrian, W., 1939, Zur Frage der Deutung der Linien im Spektrum der Sonnenkorona, Naturwissenschaften, 27, 214-214

Hale, G. E., 1908, On the Probable Existence of a Magnetic Field in Sun-Spots, ApJ, 28, 315

Hall, J. C., 2008, Stellar Chromospheric Activity, Living Reviews in Solar Physics, 5

Hannah, I. G., Kontar, E. P., 2012, Differential emission measures from the regularized inversion of Hinode and SDO data, A\&A, 539, A146, 1201.2642

Hansteen, V., De Pontieu, B., Carlsson, M., Lemen, J., Title, A., Boerner, P., Hurlburt, N., Tarbell, T. D., Wuelser, J. P., Pereira, T. M. D., De Luca, E. E., Golub, L., McKillop, S., Reeves, K., Saar, S., Testa, P., Tian, H., Kankelborg, C., Jaeggli, S., Kleint, L., Martínez-Sykora, J., 2014, The unresolved fine structure resolved: IRIS observations of the solar transition region, Science, 346, 1255757, 1412.3611 
Harvey, K. L., White, O. R., 1999, Magnetic and Radiative Variability of Solar Surface Structures. I. Image Decomposition and Magnetic-Intensity Mapping, ApJ, 515, 812831

Heyvaerts, J., Priest, E. R., 1992, A self-consistent turbulent model for solar coronal heating, ApJ, 390, 297-308

Hollweg, J. V., 1991, Alfvén Waves, in Mechanisms of Chromospheric and Coronal Heating, (Eds.) P. Ulmschneider, E. R. Priest, R. Rosner, p. 260

Innes, D. E., 2001, Coordinated observations of the quiet Sun transition region using SUMER spectra, TRACE images and MDI magnetograms, A\&A, 378, 1067-1077

Inverarity, G. W., Priest, E. R., 1995, Turbulent coronal heating. III. Wave heating in coronal loops., A\&A, 302, 567

Ishikawa, R., Tsuneta, S., 2009, Properties of Transient Horizontal Magnetic Fields, in The Second Hinode Science Meeting: Beyond Discovery-Toward Understanding, (Eds.) B. Lites, M. Cheung, T. Magara, J. Mariska, K. Reeves, vol. 415 of Astronomical Society of the Pacific Conference Series, p. 132

Ishikawa, R., Tsuneta, S., Ichimoto, K., Isobe, H., Katsukawa, Y., Lites, B. W., Nagata, S., Shimizu, T., Shine, R. A., Suematsu, Y., Tarbell, T. D., Title, A. M., 2008, Transient horizontal magnetic fields in solar plage regions, A\&A, 481, L25-L28, 0802.1769

Ishikawa, R., Tsuneta, S., Jurčák, J., 2010, Three-Dimensional View of Transient Horizontal Magnetic Fields in the Photosphere, ApJ, 713, 1310-1321, 1003.1376

Jafarzadeh, S., Cameron, R. H., Solanki, S. K., Pietarila, A., Feller, A., Lagg, A., Gandorfer, A., 2014, Migration of Ca II H bright points in the internetwork, A\&A, 563, A101, 1401.7522

Kerr, G. S., Simões, P. J. A., Qiu, J., Fletcher, L., 2015, IRIS observations of the Mg ii h and $\mathrm{k}$ lines during a solar flare, A\&A, 582, A50, 1508.03813

Klimchuk, J. A., 2006, On Solving the Coronal Heating Problem, Sol. Phys., 234, 41-77, astro-ph/0511841

Kobayashi, K., Cirtain, J., Winebarger, A. R., Korreck, K., Golub, L., Walsh, R. W., De Pontieu, B., DeForest, C., Title, A., Kuzin, S., Savage, S., Beabout, D., Beabout, B., Podgorski, W., Caldwell, D., McCracken, K., Ordway, M., Bergner, H., Gates, R., McKillop, S., Cheimets, P., Platt, S., Mitchell, N., Windt, D., 2014, The HighResolution Coronal Imager (Hi-C), Sol. Phys., 289, 4393-4412

Krivova, N. A., Solanki, S. K., Fligge, M., Unruh, Y. C., 2003, Reconstruction of solar irradiance variations in cycle 23: Is solar surface magnetism the cause?, A\&A, 399, L1-L4 
Lagg, A., Solanki, S. K., Riethmüller, T. L., Martínez Pillet, V., Schüssler, M., Hirzberger, J., Feller, A., Borrero, J. M., Schmidt, W., del Toro Iniesta, J. C., Bonet, J. A., Barthol, P., Berkefeld, T., Domingo, V., Gandorfer, A., Knölker, M., Title, A. M., 2010, Fully Resolved Quiet-Sun Magnetic flux Tube Observed with the SUNRISE/IMAX Instrument, ApJ, 723, L164-L168, 1009.0996

Landi, E., Feldman, U., 2008, The Thermal Structure of an Active Region Observed Outside the Solar Disk, ApJ, 672, 674-683

Leenaarts, J., Pereira, T. M. D., Carlsson, M., Uitenbroek, H., De Pontieu, B., 2013a, The Formation of IRIS Diagnostics. I. A Quintessential Model Atom of Mg II and General Formation Properties of the Mg II h \& k Lines, ApJ, 772, 89, 1306.0668

Leenaarts, J., Pereira, T. M. D., Carlsson, M., Uitenbroek, H., De Pontieu, B., 2013b, The Formation of IRIS Diagnostics. II. The Formation of the Mg II h \& k Lines in the Solar Atmosphere, ApJ, 772, 90, 1306.0671

Leighton, R. B., 1959, Observations of Solar Magnetic Fields in Plage Regions., ApJ, 130,366

Lemaire, P., Emerich, C., Curdt, W., Schuehle, U., Wilhelm, K., 1998, Solar H i Lyman alpha full disk profile obtained with the SUMER/SOHO spectrometer, A\&A, 334, 1095-1098

Lemen, J. R., Title, A. M., Akin, D. J., Boerner, P. F., Chou, C., Drake, J. F., Duncan, D. W., Edwards, C. G., Friedlaender, F. M., Heyman, G. F., Hurlburt, N. E., Katz, N. L., Kushner, G. D., Levay, M., Lindgren, R. W., Mathur, D. P., McFeaters, E. L., Mitchell, S., Rehse, R. A., Schrijver, C. J., Springer, L. A., Stern, R. A., Tarbell, T. D., Wuelser, J.-P., Wolfson, C. J., Yanari, C., Bookbinder, J. A., Cheimets, P. N., Caldwell, D., Deluca, E. E., Gates, R., Golub, L., Park, S., Podgorski, W. A., Bush, R. I., Scherrer, P. H., Gummin, M. A., Smith, P., Auker, G., Jerram, P., Pool, P., Soufli, R., Windt, D. L., Beardsley, S., Clapp, M., Lang, J., Waltham, N., 2012, The Atmospheric Imaging Assembly (AIA) on the Solar Dynamics Observatory (SDO), Sol. Phys., 275, 17-40

Lites, B. W., Leka, K. D., Skumanich, A., Martinez Pillet, V., Shimizu, T., 1996, SmallScale Horizontal Magnetic Fields in the Solar Photosphere, ApJ, 460, 1019

Loukitcheva, M., Solanki, S. K., White, S. M., 2009, The relationship between chromospheric emissions and magnetic field strength, A\&A, 497, 273-285, 0910.1985

Mariska, J. T., 1992, The solar transition region

Martínez González, M. J., Bellot Rubio, L. R., 2009, Emergence of Small-scale Magnetic Loops Through the Quiet Solar Atmosphere, ApJ, 700, 1391-1403, 0905.2691

Martínez González, M. J., Manso Sainz, R., Asensio Ramos, A., Bellot Rubio, L. R., 2010, Small Magnetic Loops Connecting the Quiet Surface and the Hot Outer Atmosphere of the Sun, ApJ, 714, L94-L97, 1003.1255 
Martínez Pillet, V., Del Toro Iniesta, J. C., Álvarez-Herrero, A., Domingo, V., Bonet, J. A., González Fernández, L., López Jiménez, A., Pastor, C., Gasent Blesa, J. L., Mellado, P., Piqueras, J., Aparicio, B., Balaguer, M., Ballesteros, E., Belenguer, T., Bellot Rubio, L. R., Berkefeld, T., Collados, M., Deutsch, W., Feller, A., Girela, F., Grauf, B., Heredero, R. L., Herranz, M., Jerónimo, J. M., Laguna, H., Meller, R., Menéndez, M., Morales, R., Orozco Suárez, D., Ramos, G., Reina, M., Ramos, J. L., Rodríguez, P., Sánchez, A., Uribe-Patarroyo, N., Barthol, P., Gandorfer, A., Knoelker, M., Schmidt, W., Solanki, S. K., Vargas Domínguez, S., 2011, The Imaging Magnetograph eXperiment (IMaX) for the Sunrise Balloon-Borne Solar Observatory, Sol. Phys., 268, 57-102, 1009.1095

Morton, R. J., McLaughlin, J. A., 2014, High-resolution Observations of Active Region Moss and its Dynamics, ApJ, 789, 105, 1405.5694

Nindos, A., Zirin, H., 1998, The Relation of CA II K Features to Magnetic Field, Sol. Phys., 179, 253-268

O’Dwyer, B., Del Zanna, G., Mason, H. E., Weber, M. A., Tripathi, D., 2010, SDO/AIA response to coronal hole, quiet Sun, active region, and flare plasma, A\&A, 521, A21

Oranje, B. J., 1986, Magnetic structure in cool stars. IX - Ultraviolet emission lines from chromospheres and transition regions, A\&A, 154, 185-196

Ortiz, A., Rast, M., 2005, How good is the Ca II K as a proxy for the magnetic flux?, Mem. Soc. Astron. Italiana, 76, 1018

Parker, E. N., 1972, Topological Dissipation and the Small-Scale Fields in Turbulent Gases, ApJ, 174, 499

Pereira, T. M. D., Leenaarts, J., De Pontieu, B., Carlsson, M., Uitenbroek, H., 2013, The Formation of IRIS Diagnostics. III. Near-ultraviolet Spectra and Images, ApJ, 778, 143, 1310.1926

Pesnell, W. D., Thompson, B. J., Chamberlin, P. C., 2012, The Solar Dynamics Observatory (SDO), Sol. Phys., 275, 3-15

Peter, H., 2000, Multi-component structure of solar and stellar transition regions, A\&A, $360,761-776$

Peter, H., 2001, On the nature of the transition region from the chromosphere to the corona of the Sun, A\&A, 374, 1108-1120

Peter, H., 2013, Magnetic Field Diagnostics and Spatio-Temporal Variability of the Solar Transition Region, Sol. Phys., 288, 531-547, 1303.1825

Peter, H., Gudiksen, B. V., Nordlund, A., 2006, Forward Modeling of the Corona of the Sun and Solar-like Stars: From a Three-dimensional Magnetohydrodynamic Model to Synthetic Extreme-Ultraviolet Spectra, ApJ, 638, 1086-1100, astro-ph/0503342 
Peter, H., Bingert, S., Klimchuk, J. A., de Forest, C., Cirtain, J. W., Golub, L., Winebarger, A. R., Kobayashi, K., Korreck, K. E., 2013, Structure of solar coronal loops: from miniature to large-scale, A\&A, 556, A104, 1306.4685

Peter, H., Tian, H., Curdt, W., Schmit, D., Innes, D., De Pontieu, B., Lemen, J., Title, A., Boerner, P., Hurlburt, N., Tarbell, T. D., Wuelser, J. P., Martínez-Sykora, J., Kleint, L., Golub, L., McKillop, S., Reeves, K. K., Saar, S., Testa, P., Kankelborg, C., Jaeggli, S., Carlsson, M., Hansteen, V., 2014, Hot explosions in the cool atmosphere of the Sun, Science, 346, 1255726, 1410.5842

Pevtsov, A. A., Fisher, G. H., Acton, L. W., Longcope, D. W., Johns-Krull, C. M., Kankelborg, C. C., Metcalf, T. R., 2003, The Relationship Between X-Ray Radiance and Magnetic Flux, ApJ, 598, 1387-1391

Porter, J. G., Moore, R. L., Reichmann, E. J., Engvold, O., Harvey, K. L., 1987, Microflares in the solar magnetic network, ApJ, 323, 380-390

Press, W. H., Teukolsky, S. A., Vetterling, W. T., Flannery, B. P., 2002, Numerical recipes in $\mathrm{C}++$ : the art of scientific computing

Rathore, B., Carlsson, M., Leenaarts, J., De Pontieu, B., 2015, The Formation of IRIS Diagnostics. VI. The Diagnostic Potential of the C II Lines at $133.5 \mathrm{~nm}$ in the Solar Atmosphere, ApJ, 811, 81, 1508.04423

Reale, F., 2010, Coronal Loops: Observations and Modeling of Confined Plasma, Living Reviews in Solar Physics, 7, 5, 1010.5927

Reeves, E. M., Timothy, J. G., Huber, M. C. E., 1977, Extreme UV spectroheliometer on the Apollo Telescope Mount, Appl. Opt., 16, 837-848

Régnier, S., Alexander, C. E., Walsh, R. W., Winebarger, A. R., Cirtain, J., Golub, L., Korreck, K. E., Mitchell, N., Platt, S., Weber, M., De Pontieu, B., Title, A., Kobayashi, K., Kuzin, S., DeForest, C. E., 2014, Sparkling Extreme-ultraviolet Bright Dots Observed with Hi-C, ApJ, 784, 134, 1402.2457

Reiners, A., 2012, Observations of Cool-Star Magnetic Fields, Living Reviews in Solar Physics, 9, 1, 1203.0241

Rezaei, R., Schlichenmaier, R., Beck, C. A. R., Bruls, J. H. M. J., Schmidt, W., 2007, Relation between photospheric magnetic field and chromospheric emission, A\&A, 466, 1131-1144, astro-ph/0701896

Rouppe van der Voort, L. H. M., Rutten, R. J., Vissers, G. J. M., 2016, Reconnection brightenings in the quiet solar photosphere, A\&A, 592, A100, 1606.03675

Rutten, R. J., Uitenbroek, H., 1991, CA II H(2v) and K(2v) cell grains, Sol. Phys., 134, $15-71$

Rybicki, G. B., Lightman, A. P., 1979, Radiative processes in astrophysics 
Sasso, C., Andretta, V., Spadaro, D., Susino, R., 2012, Solar low-lying cool loops and their contribution to the transition region EUV output, A\&A, 537, A150, 1112.0309

Scherrer, P. H., Schou, J., Bush, R. I., Kosovichev, A. G., Bogart, R. S., Hoeksema, J. T., Liu, Y., Duvall, T. L., Zhao, J., Title, A. M., Schrijver, C. J., Tarbell, T. D., Tomczyk, S., 2012, The Helioseismic and Magnetic Imager (HMI) Investigation for the Solar Dynamics Observatory (SDO), Sol. Phys., 275, 207-227

Schmit, D., Bryans, P., De Pontieu, B., McIntosh, S., Leenaarts, J., Carlsson, M., 2015, Observed Variability of the Solar Mg II h Spectral Line, ApJ, 811, 127, 1508.04714

Schrijver, C. J., 1987, Magnetic structure in cool stars. XI - Relations between radiative fluxes measuring stellar activity, and evidence for two components in stellar chromospheres, A\&A, 172, 111-123

Schrijver, C. J., 1992, The basal and strong-field components of the solar atmosphere, A\&A, 258, 507-520

Schrijver, C. J., Cote, J., Zwaan, C., Saar, S. H., 1989, Relations between the photospheric magnetic field and the emission from the outer atmospheres of cool stars. I - The solar CA II K line core emission, ApJ, 337, 964-976

Schwabe, M., 1844, Sonnenbeobachtungen im Jahre 1843. Von Herrn Hofrath Schwabe in Dessau, Astronomische Nachrichten, 21, 233

Schwarzschild, M., 1948, On Noise Arising from the Solar Granulation., ApJ, 107, 1

Shore, S. N., 2002, The Tapestry of Modern Astrophysics

Skumanich, A., Smythe, C., Frazier, E. N., 1975, On the statistical description of inhomogeneities in the quiet solar atmosphere. I - Linear regression analysis and absolute calibration of multichannel observations of the $\mathrm{Ca} /+$ / emission network, ApJ, 200, 747764

Solanki, S. K., Steiner, O., 1990, How magnetic is the solar chromosphere?, A\&A, 234, 519-529

Solanki, S. K., Steiner, O., Uitenbroeck, H., 1991, Two-dimensional models of the solar chromosphere. I - The CA II K line as a diagnostic: 1.5-D radiative transfer, A\&A, 250, 220-234

Solanki, S. K., Barthol, P., Danilovic, S., Feller, A., Gandorfer, A., Hirzberger, J., Riethmüller, T. L., Schüssler, M., Bonet, J. A., Martínez Pillet, V., del Toro Iniesta, J. C., Domingo, V., Palacios, J., Knölker, M., Bello González, N., Berkefeld, T., Franz, M., Schmidt, W., Title, A. M., 2010, SUNRISE: Instrument, Mission, Data, and First Results, ApJ, 723, L127-L133, 1008.3460

Spruit, H. C., 1979, Convective collapse of flux tubes, Sol. Phys., 61, 363-378

Stix, M., 2004, The sun : an introduction 
Sturrock, P. A., Uchida, Y., 1981, Coronal heating by stochastic magnetic pumping, ApJ, 246, 331-336

Testa, P., De Pontieu, B., Martínez-Sykora, J., DeLuca, E., Hansteen, V., Cirtain, J., Winebarger, A., Golub, L., Kobayashi, K., Korreck, K., Kuzin, S., Walsh, R., DeForest, C., Title, A., Weber, M., 2013, Observing Coronal Nanoflares in Active Region Moss, ApJ, 770, L1, 1305.1687

Tian, H., Kleint, L., Peter, H., Weber, M., Testa, P., DeLuca, E., Golub, L., Schanche, N., 2014, Observations of Subarcsecond Bright Dots in the Transition Region above Sunspots with the Interface Region Imaging Spectrograph, ApJ, 790, L29, 1407.1060

Tsuneta, S., Ichimoto, K., Katsukawa, Y., Nagata, S., Otsubo, M., Shimizu, T., Suematsu, Y., Nakagiri, M., Noguchi, M., Tarbell, T., Title, A., Shine, R., Rosenberg, W., Hoffmann, C., Jurcevich, B., Kushner, G., Levay, M., Lites, B., Elmore, D., Matsushita, T., Kawaguchi, N., Saito, H., Mikami, I., Hill, L. D., Owens, J. K., 2008, The Solar Optical Telescope for the Hinode Mission: An Overview, Sol. Phys., 249, 167-196, Q711.1715

Vernazza, J. E., Avrett, E. H., Loeser, R., 1981, Structure of the solar chromosphere. III Models of the EUV brightness components of the quiet-sun, ApJS, 45, 635-725

Wang, Y.-M., 2016, The Ubiquitous Presence of Looplike Fine Structure inside Solar Active Regions, ApJ, 820, L13

Warren, H. P., Winebarger, A. R., 2000, Small Scale Structure in the Solar Transition Region, ApJ, 535, L63-L66

Wedemeyer-Böhm, S., Lagg, A., Nordlund, Å., 2009, Coupling from the Photosphere to the Chromosphere and the Corona, Space Sci. Rev., 144, 317-350, 0809.0987

Wiegelmann, T., Solanki, S. K., 2004, Similarities and Differences between Coronal Holes and the Quiet Sun: Are Loop Statistics the Key?, Sol. Phys., 225, 227-247, 0802.0120

Wiegelmann, T., Solanki, S. K., Borrero, J. M., Peter, H., Barthol, P., Gandorfer, A., Martínez Pillet, V., Schmidt, W., Knölker, M., 2013, Evolution of the Fine Structure of Magnetic Fields in the Quiet Sun: Observations from Sunrise/IMaX and Extrapolations, Sol. Phys., 283, 253-272

Wilson, O. C., 1978, Chromospheric variations in main-sequence stars, ApJ, 226, 379_ 396

Winebarger, A. R., Walsh, R. W., Moore, R., De Pontieu, B., Hansteen, V., Cirtain, J., Golub, L., Kobayashi, K., Korreck, K., DeForest, C., Weber, M., Title, A., Kuzin, S., 2013, Detecting Nanoflare Heating Events in Subarcsecond Inter-moss Loops Using Hi-C, ApJ, 771, 21

Winebarger, A. R., Cirtain, J., Golub, L., DeLuca, E., Savage, S., Alexander, C., Schuler, T., 2014, Discovery of Finely Structured Dynamic Solar Corona Observed in the Hi-C Telescope, ApJ, 787, L10 
Zeeman, P., 1897, On the Influence of Magnetism on the Nature of the Light Emitted by a Substance., ApJ, 5, 332 


\section{Publications}

\section{Refereed publications}

Related to this thesis:

- K. Barczynski, H. Peter, S. L. Savage, Miniature loops in the solar corona, 2017, A\&A, 599, A137

Other:

- L. M. Yan, H. Peter, J. S. He, H. Tian, L. D. Xia, L. H. Wang, C. Y. Tu, L. Zhang, F. Chen, K. Barczynski, Self-Absorption in the Solar Transition Region, 2015, ApJ, 811,48

\section{Conference proceedings}

(S) 11/2013: Seminar, PMOD/WRC, Davos, Switzerland

(T) 09/2014: $14^{\text {th }}$ ESPM, Dublin, Ireland

(S) 05/2015: Friday Seminars, Astronomical Observatory, JU, Krakow, Poland

(T) 05/2015: $36^{\text {th }}$ seminar in Upice Observatory, Upice, Czech Republic

(T) 07/2015: Coronal Loop Workshop VII, Cambridge, UK

(P) 09/2015: Hinode 9 -International Science Meeting, Belfast, Northern Ireland

(T) 11/2015: Progress on EUV \& X-ray spectroscopy and imaging II, Wroclaw, Poland

(P) 06/2016: IRIS-6 the Chromosphere, Stockholm, Sweden

(S) 01/2017: Climat group seminar, MPS, Göttingen, Germany

T-conference talk, S-seminar talk, P-poster 



\section{Acknowledgements}

I would like to express my sincere thanks to:

- my supervisor Prof. Dr. Hardi Peter for giving me opportunity to carry out this study, advices and discussions on my research as well as helpful guidances

- my thesis advisor committee, Dr. Davina Innes and Prof. Dr. Stefan Dreizler for TAC meetings and advice on my work;

- Prof. Dr. Sami Solanki, Prof. Dr. Robert Jelle Rutten, Dr. Sabrina L. Savage for constructive discussions;

- the members of the coronal dynamics group: Dr. Sven Bingert, Dr. Joern Warnecke, Dr. Philippe Bourdin, Dr. Tijmen van Wettum, Dr. Feng Chen, Dr. Lakshmi Pradeep Chitta, Mr. Antonio Alessandro Cilla;

- the International Max-Planck Research School (IMPRS) for Solar System Science at the University of Göttingen for the PhD fellowship;

- IMPRS coordinator Dr. Sonja Schuh for her helps and IMPRS for your ideas and activity;

- Pradeep, Alessandro, Dr. Nai-Hwa Chen, Dr. Leping Li and Mrs. Limei Yan for many valuable discussions and advice;

- the directors of Upice Observatory, Dr. Eva Markova and eng. Marcel Belik;

- my master thesis supervisor dr hab. Grzegorz Michałek for our discussion in Krakow;

- my mentors, school and academic teachers, especially: Mrs. Iwona Karaś, dr hab. Andrzej Kułak and inż. Jerzy Kubisz for showing me a beauty of physics and teaching me a systematic work;

Finally, I would like to dedicate this thesis my parents Marianna and Leszek and my sister Kinga and thanks for their constant support and encouragement. 



\section{Curriculum vitae}

Name: Krzysztof Barczyński

Data of birth: 13 October 1988

Place of birth: Włoszczowa (Poland)

Nationality: Polish

Marital status: unmarried

\section{Education:}

09/2013 - 04/2017 Doctoral student

IMPRS at the University of Göttingen and

Max Planck Institute for Solar System Research, Göttingen, Germany

Thesis title: Small-scale structures in the upper solar atmosphere

Advisors: Hardi Peter, Stefan Dreizler, Davina Markiewicz-Innes

10/2011 - 07/2013 Master of Astronomy

Faculty of Physics, Astronomy and Applied Computer Science, Jagiellonian University, Krakow, Poland

Thesis title: A study of velocities of solar plumes

Advisor: Grzegorz Michałek

10/2007 - 07/2011 Bachelor of Astronomy

Faculty of Physics, Astronomy and Applied Computer Science, Jagiellonian University, Krakow, Poland 